\title{
Modell unterrichtszentrierter Schulentwicklung in Richtung personalisierten Lernens (uSpL-Modell)
}

In den letzten Jahrzehnten hat sich ein Verständnis von Schulentwicklung herausgebildet, dem zufolge verschiedene Akteurinnen und Akteure des Bildungssystems vermehrt kooperativ Entwicklungsprojekte zu initiieren haben und die Unterrichtsentwicklung sowie insbesondere das Lernen der Schülerinnen und Schüler als Ausgangs- und Zielpunkt von Entwicklungstätigkeiten aufgefasst werden (siehe Kapitel 2). Diese Merkmale eignen sich auch für die Beschreibung von Unterrichts- und Schulentwicklung in Richtung personalisierten Lernens: Schulteams passen Lernumgebungen an die personalen Voraussetzungen der Schülerinnen und Schüler an und erarbeiten Lernangebote für den Aufbau fachlicher und überfachlicher Kompetenzen, in deren Rahmen selbstgesteuertes und eigenverantwortliches Lernen möglich ist und Lehrpersonen wie auch die Lerngemeinschaft bildend wirken (siehe Kapitel 1). Vor diesem Hintergrund stellt sich die weiterführende Frage, in welchen Bereichen und wie Schulleitende und Lehrperson ihre Schule und ihren Unterricht weiterentwickeln, damit personalisiertes Lernen umgesetzt werden kann. Um dieser Frage nachzugehen, wurde für die vorliegende Arbeit das Modell $u$ nterrichtszentrierter Schulentwicklung in Richtung personalisierten Lernens (uSpL-Modell) konzipiert.

Im ersten Teil des Kapitels werden die Grundlagen des uSpL-Modells (Abschnitt 4.1) und das Modell in seinen Bestandteilen beschrieben (Abschnitt 4.2). Des Weiteren wird der Fokus auf die Einzelschule und den Unterricht kontextualisiert (Abschnitt 4.3). Anschließend werden die sieben Bereiche des Modells detailliert erläutert: Entwicklungsauslöser (Abschnitt 4.4), Tätigkeitsfelder (Entwicklungsdimensionen) (Abschnitt 4.5), Formen der Prozessgestaltung (Abschnitt 4.6), innerschulische Entwicklungsgruppen (Abschnitt 4.7), außerschulische Entwicklungsgruppen (Abschnitt 4.8), Rahmenbedingungen (Abschnitt 4.9) und Metaziele des Entwicklungsprozesses (Abschnitt 4.10). 


\subsection{Grundlagen des Modells unterrichtszentrierter Schulentwicklung in Richtung personalisierten Lernens (uSpL-Modell)}

Die Konzeption des uSpL-Modells geht davon aus, dass die Einführung und die Weiterentwicklung von Konzepten personalisierten Lernens und die über den Unterricht hinausgehenden Veränderungen innerhalb der Einzelschule als Prozess beschreibbar sind: Durch vernetztes Handeln vor allem von Schulleitenden und Lehrpersonen werden verschiedene Tätigkeitsfelder, sogenannte Entwicklungsdimensionen, so gestaltet, dass in den Lernumgebungen vermehrt kooperatives und individualisiertes Lernen ermöglicht wird und die Schülerinnen und Schüler über mehr Autonomie- und Handlungsspielräume in ihren Lernprozessen verfügen, als dies in traditionellen Unterrichtsformen der Fall ist. Der prozesshafte Charakter zeigt sich auch in Entwicklungsphasen: Die Entwicklung in Richtung personalisierten Lernens wird durch Auslöser, wie zum Beispiel heterogenere Lerngruppen, angestoßen und Unterrichtskonzepte werden hinsichtlich übergeordneter (Meta-)Ziele entwickelt, erprobt, reflektiert, angepasst und gefestigt.

Konzipiert und in seinen Bestandteilen fortlaufend ausgearbeitet wurde das uSpL-Modell in einem wechselseitigen Zusammenspiel zwischen theoretischen Analysen von Konzepten und der empirischen Datenanalyse in dieser Arbeit. Es dient in erster Linie als Modell für die Analyse empirischer Daten, auf dessen Grundlage zum Beispiel Kategoriensysteme oder Arbeitshypothesen erarbeitet werden können. Sodann besteht der heuristische Wert des Modells darin, dass empirische Befunde und weitere Themen hinsichtlich der Unterrichts- und Schulentwicklung in Einzelschulen eingeordnet und in Relation zur pädagogischpsychologischen Unterrichtsforschung sowie zur Schulentwicklungsforschung gesetzt werden können.

Im uSpL-Modell werden erstens am Entwicklungsprozess beteiligte Personen - allen voran Schulleitende und Lehrpersonen - und deren Kooperationsbeziehungen dargestellt. Zweitens werden Tätigkeiten, die innerhalb der Entwicklungsprozesse für die Einführung und die Weiterentwicklung von Konzepten personalisierten Lernens relevant sind, in Entwicklungsdimensionen zusammengefasst und drittens wird der Prozessverlauf in Phasen abgebildet. In Anbetracht dieser drei Inhalte stellt das uSpL-Modell einen Versuch dar, die äußerst komplexen Abläufe in Entwicklungsprozessen zu systematisieren und Beschreibungsund Erklärungsansätze für Entwicklungsprozesse in Richtung personalisierten Lernens anzubieten. Aus diesem Grund besteht die Möglichkeit, das Modell auch 
über die in dieser Arbeit untersuchten Schulen auf andere Schulen anzuwenden und beispielsweise deren Entwicklungsprozess zu reflektieren und positive sowie herausfordernde Aspekte zu eruieren. Allerdings wäre hierfür eine nicht zu unterschätzende Transferleistung notwendig.

Zusammenfassend gründet das uSpL-Modell auf den folgenden vier Bedingungen einer Schulentwicklungstheorie nach Maag Merki (2008):

- Strukturanalyse: Es werden wesentliche Entwicklungsdimensionen sowie Akteursgruppen von Schulentwicklung und ihre Beziehung zueinander benannt. Hierbei werden Ziele und Aufgaben von Schulen mit Konzepten personalisierten Lernens sowie die Bedingungsfaktoren (personalisierter) Bildungsprozesse der Schülerinnen und Schüler berïcksichtigt.

- Prozess- und Entwicklungsanalyse: Es werden Prozesse und Entwicklungstätigkeiten zur Professionalisierung relevanter Akteursgruppen hinsichtlich ihres schulischen Handelns in den einzelnen Entwicklungsdimensionen beschrieben.

- Wirkungsanalyse: Voraussetzungen und Bedingungsfaktoren der relevanten Strukturdimensionen und deren Entwicklung werden dargestellt.

- Hypothesenbildung und empirische Überprüfung: Das uSpL-Modell kann für weitere Studien als theoretische und analytische Grundlage genutzt werden. In diesem Zusammenhang ist eine empirische Überprüfung des Modells möglich, indem Hypothesen formuliert werden, nach deren Klärung das Modell gegebenenfalls modifiziert werden kann.

Die dem uSpL-Modell zugrunde liegenden Bezugstheorien sind, wie bereits erwähnt, einerseits Theorien der pädagogisch-psychologischen Lehr- und Lernforschung (u. a. Reusser, 2011) und andererseits Schulentwicklungstheorien bezüglich der Entwicklung von Einzelschulen (u. a. Maag Merki, 2017; Rolff, 2013). Diese werden im Modell zusammengeführt. Auf diese Weise werden Beschreibungen der Entwicklungsprozesse in Bezug zu qualitätsvollen Lernumgebungen gestellt, die tiefenverstehendes und nachhaltiges Lernen der Schülerinnen und Schüler ermöglichen können. Hinsichtlich der pädagogisch-psychologischen Unterrichtsforschung war bei der Erarbeitung des uSpL-Modells die folgende Frage leitend: Was zeichnet qualitätsvolle, personalisierte Unterrichtskonzepte aus und wie können diese im Hinblick auf die pädagogischen und didaktischen Tätigkeiten des Lehrens und Lernens wirksam implementiert und weiterentwickelt werden (siehe Abschnitt 4.5.1)? Diesbezüglich wurde angenommen, dass Entwicklungstätigkeiten auf der Lehr- und Lernebene wirksam werden müssen, damit sich Lernprozesse und der Lernerfolg der Schülerinnen und Schüler nachhaltig verbessern lassen. Bei den Schulentwicklungstheorien standen aus 
einer handlungs- und akteurszentrierten Perspektive Fragen nach den beteiligten Akteursgruppen (siehe Abschnitt 4.7 und Abschnitt 4.8) nach ihren Tätigkeitsfeldern (siehe Abschnitt 4.5) sowie danach, wie diese Personen die Entwicklungsprozesse kooperativ gestalten, im Zentrum (siehe Abschnitt 4.6). Darüber hinaus beziehen sich die nachfolgenden Ausführungen auf Schultheorien, auf deren Grundlage die Fragen geklärt werden können, worin die Rahmenbedingungen von Schulen bestehen (siehe Abschnitt 4.9), was zentrale Bestimmungsfaktoren von Einzelschulen sind und was diese als pädagogische Handlungseinheit (Fend, 1986) charakterisiert (siehe Abschnitt 4.3). Auch Schuleffektivitätstheorien werden mitaufgenommen, und zwar im Zusammenhang mit der folgenden Frage: Was sind Metaziele einer unterrichtszentrierten Schulentwicklung in Richtung personalisierten Lernens (siehe Abschnitt 4.10)? In der Modellbeschreibung wird fortlaufend auf diese Leitfragen Bezug genommen. Anschließend wird in der Zusammenfassung der theoretischen Ausführungen (siehe Kapitel 5) versucht, sie zu beantworten.

\subsection{Schaubild des Modells unterrichtszentrierter Schulentwicklung in Richtung personalisierten Lernens (uSpL-Modell)}

Das uSpL-Modell (Abbildung 4.1) ist in drei Hauptsegmente unterteilt: mögliche Auslöser einer Entwicklung, den Entwicklungsprozess selbst sowie Metaziele. Im Folgenden werden diese Segmente kurz beschrieben, wobei für die ausführlichen Erläuterungen auf das dazugehörige Kapitel verwiesen wird.

Mögliche Auslöser einer unterrichtszentrierten Schulentwicklung in Richtung personalisierten Lernens

Das erste Hauptsegment sind potenzielle Entwicklungsauslöser (siehe Abschnitt 4.4) in Richtung personalisierten Lernens. Dazu gehören zum Beispiel die folgenden:

- heterogener werdende Lerngruppen,

- reformpädagogische Bestrebungen eines kindorientierten Unterrichts,

- die Möglichkeiten und Potenziale von Lern- und Verwaltungssoftwares (ICT) zur Unterstützung individuellen Lernens,

- Bestrebungen, neben fachlichen vermehrt auch überfachliche Kompetenzen auszubilden, 


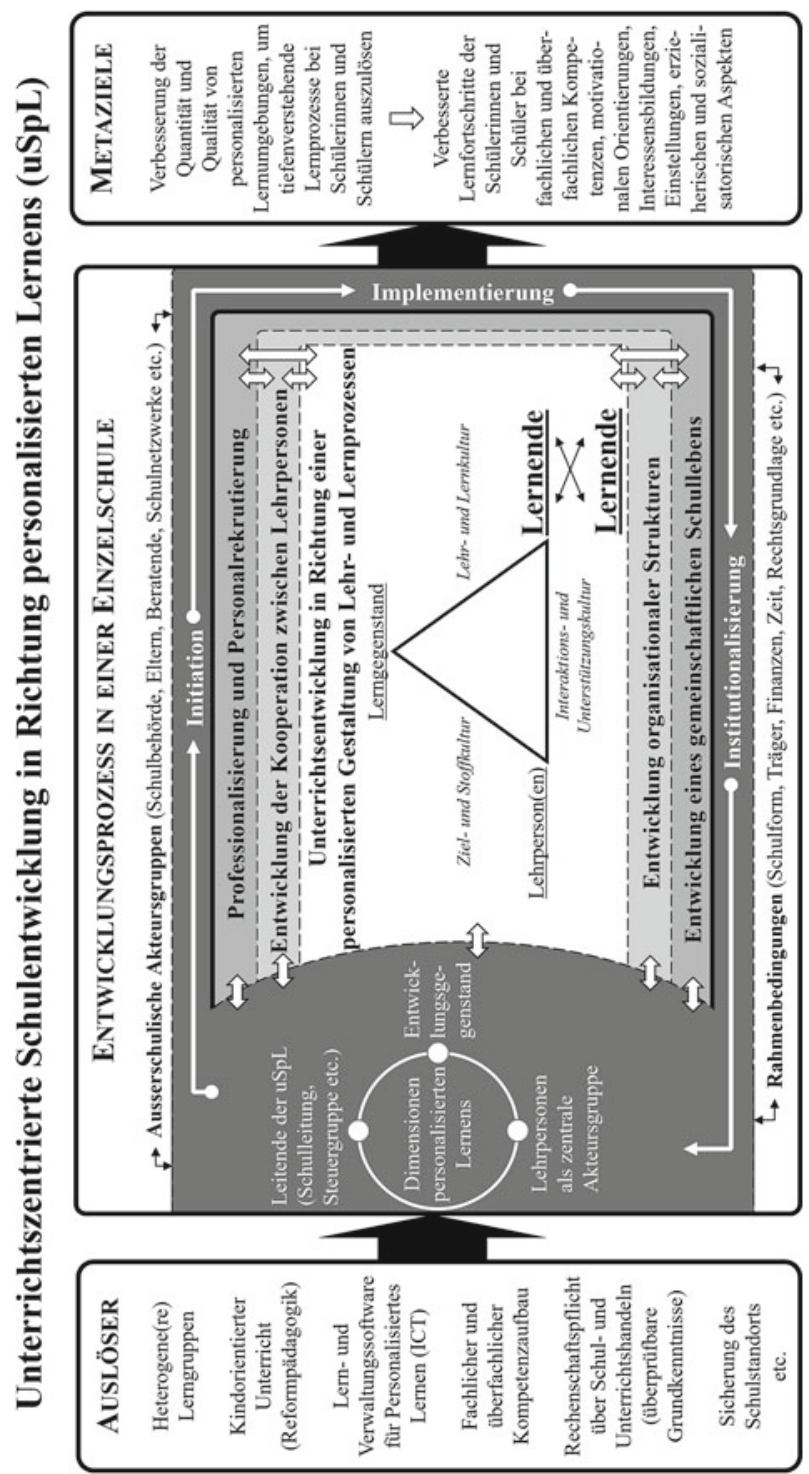

Abbildung 4.1 uSpL-Modell (eigene Darstellung): unterrichtszentrierte Schulentwicklung in Richtung personalisierten Lernens (u. a. in Anlehnung u. a. an: Bastian, 2007; Bray \& McClaskey, 2015; Ditton, 2000; Giaquinta, 1973; D. Hargreaves, 2006a; S. G. Huber \& Büeler, 2009; Prain et al., 2015a; Reusser \& Pauli, 2010b; Reusser et al., 2013; Rolff, 2007; Stebler et al., 2018) 
- die zunehmende Rechenschaftspflicht von Schulleitenden und Lehrpersonen bezüglich ihrer Schul- und Unterrichtsarbeit und

- die Sicherung des Schulstandorts im Fall einer drohenden Schulschließung (u. a. Bray \& McClaskey, 2015; D. Hargreaves, 2006a; Stebler et al., 2017).

In dieser Aufzählung sind nur die in der einschlägigen Literatur am häufigsten aufgeführten Auslöser aufgeführt. Die Liste ist daher nicht abschließend und kann mit weiteren Aspekten ergänzt werden. Zudem können einzelne oder mehrere Auslöser in Kombination Schulleitende und Lehrpersonen dazu bewegen, den Entwicklungsprozess in Richtung personalisierten Lernens zu initiieren.

\section{Entwicklungsprozess in einer Einzelschule}

Im zweiten Hauptsegment ist der Entwicklungsprozess in einer Einzelschule modelliert. Hierbei werden drei Teilsegmente unterschieden: der Prozessverlauf (siehe Abschnitt 4.6), die fünf Entwicklungsdimensionen (siehe Abschnitt 4.5) und die Rahmenbedingungen (siehe Abschnitt 4.9) mit den außerschulischen Akteursgruppen (siehe Abschnitt 4.8).

Im ersten Teilsegment ist die Kooperation zwischen Leitenden der uSpL (Schulleitende, Steuergruppe etc.) und Lehrpersonen, welche die Entwicklungen in ihrem Unterricht und darüber hinausgehende Tätigkeiten umsetzen, abgebildet. Inhalt dieser Kooperation sind die Entwicklungsgegenstände, zum Beispiel die Erarbeitung von Aufgabensammlungen, die größtenteils selbstständig bearbeitet werden können, oder das Umstrukturieren von Lerngruppen (u. a. D. Hargreaves, 2006a). Diese Kooperation zielt auf die Weiterentwicklung personalisierter Lernumgebungen $a b$, welche in fünf Dimensionen beschrieben werden können: Lernumgebungen werden durch gezielte Entwicklungstätigkeiten vermehrt an die personalen Lernvoraussetzungen angepasst, fachlicher und überfachlicher Kompetenzaufbau wird gefördert, selbstständiges sowie eigenverantwortliches Lernen ist möglich und die Lehrperson und die Lerngemeinschaft wirken bildend (siehe Abschnitt 3.2, u. a. Stebler et al., 2018). Mit der Schulentwicklungsperspektive sind diese Dimensionen personalisierten Lernens nicht nur Beschreibungskategorien, sondern Ausgangs- und Zielpunkt einer lernseitig ausgerichteten unterrichtszentrierten Schulentwicklung, die von den individuellen Bedingungen der Lernprozesse sowie dem Lernverhalten der Schülerinnen und Schüler ausgeht (Agostini et al., 2018).

Der Entwicklungsprozess ist durch drei Phasen charakterisiert: In der ersten Phase werden Herausforderungen eruiert und die mögliche Umsetzung von Lösungsansätzen wird vorbereitet: Definieren von Entwicklungszielen, Einrichten einer Prozesssteuerung und Erarbeiten von Projektplänen sowie Konzepten. Diese 
Tätigkeiten werden unter „Initiation“ (Giaquinta, 1973) zusammengefasst. In der anschließenden zweiten Phase, der Implementation (Giaquinta, 1973), werden die konzipierten und geplanten Veränderungen in den Unterrichts- und Schulbetrieb eingeführt und die neuen Handlungsweisen erprobt. Regelmäßiges Reflektieren, Evaluieren und Optimieren sind typische Tätigkeiten dieser Phase. In der letzten Phase, der Institutionalisierung (Giaquinta, 1973), werden die neuen Handlungsabläufe gefestigt und automatisiert und deren Nachhaltigkeit wird überprüft. Diese Phasen sind weniger als starre Abfolge aufzufassen, sondern überlagern sich größtenteils (u. a. auch Hameyer, 2005; Holtappels, 2014).

Das zweite Teilsegment umfasst fünf Tätigkeitsfelder, sogenannte Entwicklungsdimensionen, die in der Mitte des uSpL-Modells dargestellt sind:

- Unterrichtsentwicklung in Richtung einer personalisierten Gestaltung von Lehr- und Lernprozessen (siehe Abschnitt 4.5.1),

- Entwicklung organisationaler Strukturen (siehe Abschnitt 4.5.2),

- Entwicklung der Kooperation zwischen Lehrpersonen (siehe Abschnitt 4.5.3),

- Professionalisierung und Personalrekrutierung (siehe Abschnitt 4.5.4) sowie

- Entwicklung eines gemeinschaftlichen Schullebens (siehe Abschnitt 4.5.5) (u. a. D. Hargreaves, 2006a; Prain et al., 2015b; Rolff, 2013).

Die Entwicklungsdimensionen bedingen sich gegenseitig und stehen in einem wechselseitigen Einfluss, je nachdem, auf welche in den Entwicklungstätigkeiten Bezug genommen wird. Das heißt, eine Veränderung in einer Entwicklungsdimension kann zu Veränderungen in den anderen Entwicklungsdimensionen führen. Zudem sind die Entwicklungsdimensionen hierarchisch angeordnet. Im Zentrum steht die Unterrichtsentwicklung. Darum herum ordnen sich die Entwicklung organisationaler Strukturen und die Entwicklung der Kooperation zwischen Lehrpersonen an. Anschließend folgen die Professionalisierung und die Personalrekrutierung sowie die Entwicklung eines gemeinschaftlichen Schullebens (siehe Abschnitt 4.5). Diese Hierarchisierung gründet auf den theoretischen Bezugstheorien sowie der deduktiv-induktiven Datenanalyse in Studie 1 (siehe Abschnitt 7.1). Um Entwicklungstätigkeiten bezüglich des Unterrichts systematisch untersuchen zu können, wird als theoretische Grundlage ein didaktisches Dreieck (Reusser, 1999) verwendet: An den drei Ecken sind die Lehrperson oder mehrere Lehrpersonen, die Schülerinnen und Schüler sowie der Lerngegenstand positioniert. Die Kanten beziehen sich auf drei Kulturen: Ziel- und Stoffkultur, Lehrund Lernkultur sowie Interaktions- und Beziehungskultur. Für einen nachhaltigen Entwicklungsprozess, der auf verbesserte Lernprozesse der Schülerinnen und 
Schüler abzielt, sind die Veränderungen von Entwicklungsgegenständen dieser drei Kulturen zentral (u. a. Reusser, 2011).

Das dritte und letzte Teilsegment bilden einerseits Rahmenbedingungen, wie zum Beispiel Schulform, Trägerschaft der Schule, finanzielle Möglichkeiten für Entwicklungstätigkeiten, verfügbare Zeit, gesetzliche Grundlagen, das Einzugsgebiet der Schule, Systemarchitektur, Lehrplan, Bildungsstandards, externe Evaluationen und Schulinspektionen, pädagogische Traditionen, Qualifikationen, Lehrpersonenaus- und -fortbildung etc. (Altrichter \& Maag Merki, 2016a; Maag Merki, 2008; Oelkers \& Reusser, 2008; Reusser \& Pauli, 2010b). Andererseits sind außerschulische Akteursgruppen, wie etwa die Schulbehörde, Eltern, Beratende oder Schulnetzwerke, dargestellt. Sowohl die Rahmenbedingungen als auch die außerschulischen Akteursgruppen wirken auf Entwicklungsprozesse ein.

Metaziele einer unterrichtszentrierten Schulentwicklung in Richtung personalisierten Lernens

Das letzte Hauptsegment sind die Metaziele (siehe Abschnitt 4.10). Sie unterscheiden sich von den Entwicklungszielen insofern, als sie die übergeordneten Ziele darstellen und der gesamte Entwicklungsprozess darauf abgestimmt sein sollte. Es sollen die Quantität und die Qualität von personalisierten Lernangeboten verbessert werden, um nachhaltige und auf tiefes Verstehen ausgerichtete Lernprozesse bei Schülerinnen und Schülern auszulösen. Ebenso zielt eine unterrichtszentrierte Schulentwicklung in Richtung personalisierten Lernens darauf ab, Lernfortschritte der Schülerinnen und Schüler zu verbessern. Diese umfassen fachliche und überfachliche Kompetenzen, motivationale Orientierungen, Interessenbildung, Einstellungen sowie erzieherische und sozialisatorische Aspekte (u. a. Altrichter \& Helm, 2011; Dedering, 2012; Fend, 2008b; Rahm, 2005; Rolff, 2013).

\subsection{Einzelschule und Unterricht im Fokus des uSpL-Modells: Verortung im Bildungssystem und Merkmale von Einzelschulen aus handlungstheoretischer und akteurszentrierter Perspektive}

Bevor die einzelnen Segmente des uSpL-Modells ausführlich beschrieben werden, wird das Modell im ,Systemischen Rahmenmodell von Unterrichtsqualität und -wirksamkeit“" (Reusser \& Pauli, 2010b) kontextualisiert. Schule ist ein Mehrebenensystem und Schulentwicklung findet entsprechend auf mehreren Ebenen statt 
(u. a. Maag Merki, 2008), jedoch können im uSpL-Modell nicht alle Ebenen gleichermaßen fokussiert werden. Der Schwerpunkt des uSpL-Modells liegt auf der Ebene der Einzelschule und des Unterrichts. Im Anschluss an diese Kontextualisierung werden Charakteristika von Einzelschulen aus handlungstheoretischer und akteurszentrierter Perspektive beschrieben.

Kontextualisierung des uSpL-Modells im „Systemischen Rahmenmodell von Unterrichtsqualität und -wirksamkeit“ (Reusser \& Pauli, 2010b)

In der Weiterentwicklung von Schule und Unterricht in Richtung personalisierten Lernens sind, angelehnt an das ,Systemische Rahmenmodell von Unterrichtsqualität und -wirksamkeit“" (Reusser \& Pauli, 2010b), drei Ebenen des Bildungssystems relevant: die Systemebene, die Ebene der Schule und Familie sowie die Ebene der Klassen und Individuen (Abbildung 4.2).

\section{Systemisches Rahmenmodell von Unterrichtsqualität und -wirksamkeit}

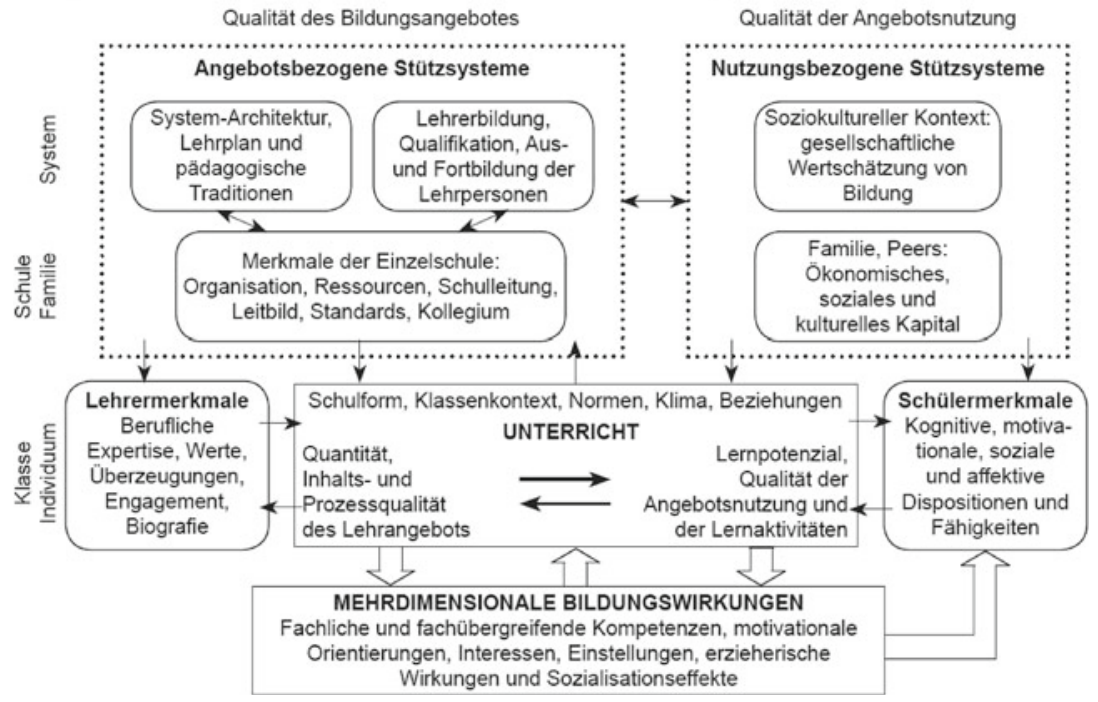

Abbildung 4.2 Systemisches Rahmenmodell von Unterrichtsqualität und -wirksamkeit (Reusser \& Pauli, 2010b)

Aus einer akteurszentrierten Perspektive sind auf der Systemebene die lokalen Schulbehörden und politische Entscheidungsträger positioniert. Auf Ebene der Schule und Familie befinden sich die Schulleitenden, das Kollegium sowie 
die Eltern und auf der Ebene der Klassen und Individuen die Lehrpersonen und die Schülerinnen und Schüler. Neben der horizontalen Differenzierung in die drei Ebenen wird im Rahmenmodell auch eine vertikale Unterscheidung zwischen angebotsbezogenen und nutzungsbezogenen Stützsystemen sowie zwischen Angebots- und Nutzungsseite des Unterrichts vorgenommen. Das uSpL-Modell fokussiert die Ebene der Schule sowie die Ebene der Klassen und Individuen: In Kooperation von Schulleitenden und dem Kollegium oder einzelnen Subteams von Lehrpersonen werden für die Schülerinnen und Schüler personalisierte Lernangebote geplant, umgesetzt und evaluiert. Hierbei ist insbesondere die didaktische und pädagogische Ausgestaltung solcher Lernumgebungen im Blickfeld. Die Kooperationsbeziehungen mit außerschulischen Akteursgruppen werden im uSpL-Modell hingegen weniger differenziert dargestellt. Ein Grund dafür liegt in den Grenzen der Darstellbarkeit von solch komplexen Kooperationen und Interaktionen zwischen allen Ebenen des Bildungssystems (u. a. Maag Merki, 2008). Ein weiterer Grund ist, dass die Entwicklung von Konzepten personalisierten Lernens im Kern die Entwicklungsarbeit von Lehrpersonen und Schulleitenden an Einzelschulen umfasst.

Neben dem Mehrebenenverständnis von Schule kann das uSpL-Modell ebenso in der Konzeption eines Angebots-Nutzungs-Modells (Fend, 2008a; Helmke, 2017; Lipowsky, 2009; Reusser \& Pauli, 2010b) kontextualisiert werden. Gemäß diesem Verständnis werden Schule und insbesondere Unterricht als eine Koproduktion von Angebotshandeln der Lehrpersonen und Nutzungshandeln der Schülerinnen und Schüler verstanden. Das Angebotshandeln der Lehrpersonen wird beispielsweise determiniert durch Vorgaben von Lerninhalten und Lernzeit, wie etwa den Lehrplan, durch individuelle Merkmale der Lehrpersonen und durch die Inhalts- und Prozessqualitäten im unterrichtlichen Handeln. Das Nutzungshandeln wiederum wird zum Beispiel durch die individuellen Merkmale der Schülerinnen und Schüler, durch deren kulturellen, sozioökonomischen und familiären Hintergrund und Lernpotenzial sowie durch die Qualitäten der Angebotsnutzung und die Lernaktivitäten beeinflusst. Die hinter dem Angebots-Nutzungs-Modell (Reusser \& Pauli, 2010b) stehende Hypothese geht davon aus, dass Koproduktion zwischen Angebots- und Nutzungshandeln verstärkte Bildungswirkungen evoziert, wenn es in der Koproduktion gelingt, kognitiv aktivierende und auf tiefes Verstehen ausgerichtete Lernprozesse bei den Schülerinnen und Schülern auszulösen. Ein solches Angebots-Nutzungs-Verständnis von Unterricht und Schule wird im uSpL-Modell in dieser Form zwar nicht explizit ersichtlich, liegt ihm aber zugrunde. So kann die Koproduktion von Angebots- und Nutzungshandeln am Beispiel der Entwicklung personalisierter Lernumgebungen aufgezeigt werden: In der Gestaltung von Lernangeboten werden verstärkt die Bedingungen und 
Merkmale des Nutzungshandelns berücksichtigt und es wird teils ein individuelles Lernangebot geschaffen. Zum Beispiel werden Lernräume entwickelt mit mehr Wahl- und Autonomiebereichen, in denen Schülerinnen und Schüler eigene Entscheidungen in ihrem Lernprozess treffen: Welche Lernaufgaben möchten sie wann und mit welchen Lernpartnerinnen oder Lernpartnern oder allein lösen? Um die Wahl- und Autonomiebereiche lernwirksam nutzen zu können, benötigen die Schülerinnen und Schüler ein Repertoire von Lernstrategien, die sie sich etwa durch angeleitetes Üben und Kultivieren in fachlich anspruchsvollen Situationen aneignen können (Reusser, 2019).

Einzelschulen mit pädagogischen und didaktischen Handlungs- und Gestaltungsspielräumen

Ein handlungs- und akteurszentriertes Verständnis von Schulentwicklung in Einzelschulen (u. a. Emmerich \& Maag Merki, 2014) geht von der Frage aus, wie Schulleitende, Lehrpersonen oder Schülerinnen und Schüler ihre Handlungen miteinander koordinieren und aufeinander abstimmen, um personalisiertes Lernen zu ermöglich. Möchten Lehrpersonen und Schulleitungen personalisierte Lernumgebungen einführen und weiterentwickeln, so benötigen sie Handlungs- und Autonomiefreiräume in der pädagogischen und didaktischen Gestaltung, damit sie ihre Schule und ihren Unterricht hinsichtlich der Zusammensetzung der Schülerinnen und Schüler und weiterer Rahmenbedingungen entsprechend anpassen können (Rolff, 1991, 1993).

Im Zuge dessen etablierte sich in den 1980er-Jahren ein Verständnis von Einzelschule als pädagogische Handlungseinheit (Fend, 1986, 2017) (siehe Abschnitt 2.1). Darunter ist zu verstehen, dass in Einzelschulen Vorgaben, beispielsweise der Systemebene, und weitere Entwicklungsimpulse rekontextualisiert werden (Fend, 2008b). Das heißt, Entwicklungsentscheidungen innerhalb einer Einzelschule werden gefällt und Entwicklungstätigkeiten durchgeführt, indem schulexterne Vorgaben (z. B. Verordnungen, Gesetze, Erwartungen Schulbehörde und Eltern etc.) an den schulinternen Handlungskontext (z. B. schulinterne Abmachungen und Beschlüsse, Heterogenität der Schülerinnen und Schüler, etablierte Unterrichtskonzepte etc.) adaptiert werden (Dalin \& Rolff, 1990; Fend, 2011; Maag Merki, 2008; Wissinger, 2014).

Dieses Verständnis gründet u. a. auf Studienergebnissen von Fend (1982) oder auch von Rutter, Maughan, Mortimer und Ouston (1980). Sie konnten aufzeigen, dass sich Schulen der gleichen Schulform hinsichtlich des Schulklimas und der Leistungsresultate häufig stärker voneinander unterscheiden als die Schulformen untereinander. Darüber hinaus konnten Chrispeels, Andrews und González (2007) 
in einer Forschungsübersicht aus dem angloamerikanischen Bildungsraum aufzeigen, dass solche Differenzen auch innerhalb einer Einzelschule zu finden sind, das heißt zwischen den Lehrpersonen respektive ihren Klassen.

\section{Konträre und widersprüchliche Handlungsziele in Schule und Unterricht}

Schulen als pädagogische Handlungseinheiten verfügen mitunter über Gestaltungsräume, die mit der Herausforderung einhergehen, dass die Handlungsziele in pädagogischen Organisationen konträr und widersprüchlich sein können (Rolff, 1991). Illustrieren lässt sich dies am Vergleich zwischen der Allokationsfunktion von Schule (Fend, 2008a) und dem beim personalisierten Lernen angestrebten Eingehen auf persönliche Lernwege (Bray \& McClaskey, 2015; Stebler et al., 2018). Die Allokationsfunktion umfasst die Zuordnung und die Selektion der Schülerinnen und Schüler im Hinblick auf ihre beruflichen Laufbahnen mittels Prüfungen und Notenvergabe. Diese Prüfungen sind standardisiert und berücksichtigen kaum, und damit anders als im personalisierten Lernen vorgesehen, persönliche Lernwege. Dies erfordert einen Rahmen, in dem mit Prüfungen und Noten auf die individuellen Fähigkeiten und Fertigkeiten eingegangen werden kann. Zugespitzt formuliert bewegen sich Lehrpersonen in Schulen mit Konzepten personalisierten Lernens somit zwischen den standardisierten Zuordnungsverfahren und dem Ermöglichen von personalen Lernwegen.

\section{Einzelschule als lose gekoppeltes System mit einem Autonomie-Paritäts-Muster}

Damit Schulleitende und Lehrpersonen die pädagogischen und didaktischen Gestaltungsfreiräume nutzen und, wie im uSpL-Modell dargestellt, beispielsweise Konzepte personalisierten Lernens einführen können, ist es erforderlich, dass Lehrpersonen vermehrt eng und intensiv miteinander kooperieren, etwa indem sie neue Lernumgebungen zeitlich und räumlich strukturieren, gemeinsam Lernaufgaben erarbeiten oder miteinander und füreinander Unterricht vorbereiten (u. a. Bray \& McClaskey, 2015; Galle, Stebler \& Reusser, 2019; Prain et al., 2015a; Stebler, Galle, Pauli \& Reusser, eingereicht). Hierfür sind Organisationsstrukturen nötig, bei denen Einzelpersonen und Teams eng miteinander verknüpft sind und ihre Handlungen aufeinander abstimmen.

Ein solches Verständnis von Schule ist historisch eher die Ausnahme. Schulen sind heute noch eher lose gekoppelte Systeme (Weick, 1982), in denen Arbeitsbereiche und -prozesse wenig ineinandergreifen und Lehrpersonen schwach miteinander vernetzt sind. Das ist beispielsweise daran erkennbar, dass Lehrpersonen mit ihren Klassen eher unabhängig voneinander fungieren. Im Vergleich zu einer Fabrik, bei der bestimmte Arbeitsprozesse für das fertige Produkt aufeinander aufbauen, ist dies bei Klassen in einer Einzelschule eher nicht der Fall: 
Hier ist jede Klasse für sich unabhängig und ihre Erfolge und Misserfolge beeinflussen kaum den Unterricht einer anderen Klasse. Der lose Arbeitsverbund wird auch in der Kooperation unter Lehrpersonen deutlich. In diesem Zusammenhang etablierte sich der Begriff eines Autonomie-Paritäts-Musters (Lortie, 1975), mit dem sich das meist autonome Handeln von Lehrpersonen charakterisieren lässt. Dieses autonome Handeln manifestiert sich u. a. darin, dass sich Lehrpersonen in ihrem Handeln in der Regel nur wenig gegenseitig beeinflussen. Des Weiteren betrachten sie sich als gleichwertige Partnerinnen und Partner, was dazu führt, dass das gegenseitige Einmischen in Tätigkeiten anderer eher unerwünscht ist. Kritik und Anregungen für den Unterricht von Kolleginnen und Kollegen werden häufig als unangebracht erachtet (u. a. Altrichter \& Eder, 2004; Eder, Dämon \& Hörl, 2011).

\section{Begrenzte Technologisierbarkeit von Schule und Unterricht}

Eine intensive Kooperation in personalisierten Unterrichtskonzepten ist auch für die Bearbeitung von komplexen Herausforderungen in pädagogischen Feldern, bei denen es keine standardisierten Lösungen gibt, hilfreich. Pädagogisches und didaktisches Handeln werden als begrenzt technologisierbar beschrieben (Feindt, Fichten \& Meyer, 2006). Beim Bearbeiten von Herausforderungen können die Beteiligten nur in begrenztem Masse auf technokratische Handlungslogiken zurückgreifen, die auf monokausalen Schrittfolgen und Zusammenhängen beruhen, sondern sie müssen in einem komplexen, multidimensionalen Feld verschiedene Handlungsmöglichkeiten gegeneinander abwägen (Lipowsky, 2014). Hieraus folgt eine immanente Kontrollunsicherheit (Rolff, 1991), welche sich auf die permanente Unsicherheit bezieht, ob die Umsetzung von Lösungsansätzen erfolgreich ist oder sein kann. Zum einen können Herausforderungen und Probleme je nach Erfahrungs- und Wissensbeständen von verschiedenen Personen unterschiedlich definiert werden (ill-defined problems) und zum anderen fehlen Standardrezepte für spezifisch didaktische und pädagogische Situationen weitgehend. Deshalb sind Lösungsansätze für pädagogische und didaktische Herausforderungen immer abhängig von der Situation, den beteiligten Personen sowie den Rahmenbedingungen (Klafki, 1985/2007; Rolff, 2013; Stern, 2009). 


\subsection{Mögliche Auslöser einer unterrichtszentrierten Schulentwicklung in Richtung personalisierten Lernens}

In den vorherigen Unterkapiteln wurden die Grundlagen des uSpL-Modells und die einzelnen Hauptsegmente beschrieben. Nun werden die einzelnen Segmente, beginnend mit potenziellen Auslösern einer unterrichtszentrierten Schulentwicklung in Richtung personalisierten Lernens, detailliert erläutert.

Auslöser sind die Beweggründe und Treiber einer Entwicklung und markieren einen Ausgangspunkt für Veränderungen. Verschiedene Veränderungen in der Gesellschaft - zum Beispiel die zunehmende Digitalisierung - und im Bildungssystem - zum Beispiel die integrativen/inklusiven Schulformen - können dazu führen, dass Schulleitende und Lehrpersonen mit der Schul- und Unterrichtssituation unzufrieden sind, weil das Lernangebot und dessen Nutzung durch die Schülerinnen und Schüler nicht ihren Erwartungen und ihren Vorstellungen entsprechen. Dies kann sie dazu veranlassen, ihre bisherigen Handlungsweisen zu hinterfragen und Entwicklungsmöglichkeiten abzuwägen.

Am Anfang einer unterrichtszentrierten Schulentwicklung in Richtung personalisierten Lernens können ein oder mehrere Auslöser stehen (Abbildung 4.3), beispielsweise zunehmend heterogenere Lerngruppen, reformpädagogische Bestrebungen eines kindorientierten Unterrichts, Lern- und Verwaltungssoftwares (ICT), stärkere Gewichtung des Aufbaus überfachlicher Kompetenzen, Rechenschaftspflicht bezüglich des Schul- und Unterrichtshandelns sowie Sicherung des Schulstandorts. Diese Auflistung ist nicht abschließend, gibt aber wie bereits angemerkt die in der Literatur (u. a. Bray \& McClaskey, 2015; D. Hargreaves, 2006a; Stebler et al., 2017) am umfassendsten beschriebenen Auslöser wieder.

Systematisierung möglicher Auslöser in schulinterne und schulexterne Auslöser

Die aufgeführten Auslöser lassen sich aus einer akteurszentrierten Perspektive in schulinterne und schulexterne Auslöser unterscheiden. Durch schulinterne Auslöser werden Entwicklungsprozesse von Lehrpersonen und/oder Schulleitenden initiiert, die auf eine Reflexion und Analyse der eigenen Schul- und Unterrichtssituation zurückzuführen sind. Wie bereits oben beschrieben, können die Beteiligten beispielsweise unzufrieden mit der Unterrichtssituation sein, weil ihre Lernangebote von den Schülerinnen und Schülern nicht wie erwartet genutzt werden. Schulexterne Auslöser hingegen sind durch außerschulische Akteursgruppen - etwa Schulbehörde, Personen der Bildungspolitik, Eltern etc. 


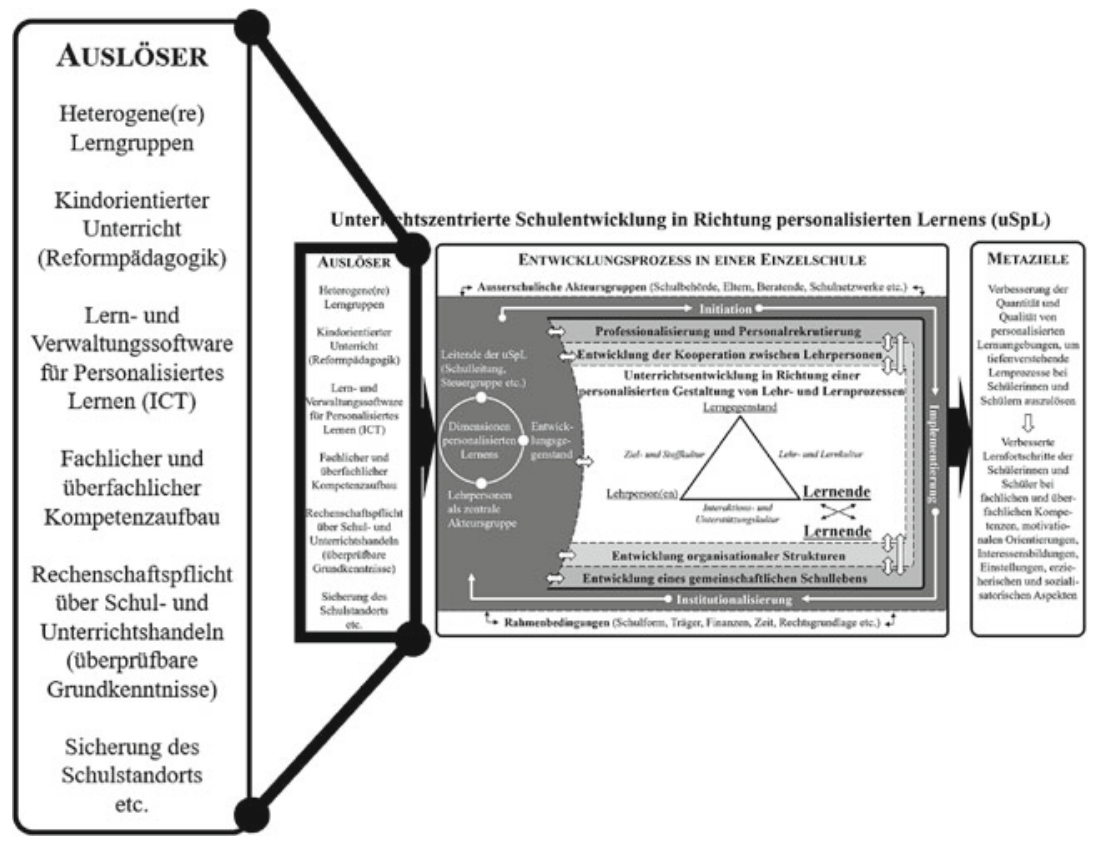

Abbildung 4.3 Mögliche Auslöser einer unterrichtszentrierten Schulentwicklung in Richtung personalisierten Lernens (eigene Darstellung)

- gesetzte Entwicklungsimpulse, zum Beispiel von Schulbehörden geforderte Entwicklungsprojekte oder eine angekündigte Schließung einer Schule aufgrund der geringen Anzahl an Schülerinnen und Schülern (Kiper, 2013; Kurz \& Weiß, 2016; Schratz \& Steiner-Löffler, 1999). In welcher Weise sich die internen und externen Entwicklungsauslöser gegenseitig bedingen und empirisch abgrenzen lassen, ist noch wenig erforscht (Emmerich \& Maag Merki, 2014). Die nachfolgend beschriebenen Auslöser einer unterrichtszentrierten Schulentwicklung in Richtung personalisierten Lernens sind daher je nachdem, welche Personen Veränderungen anregen und fordern, als schulinterner und/oder als schulexterner Auslöser beschreibbar.

\section{Heterogene Zusammensetzung der Schülerinnen und Schüler}

Zunehmende Globalisierung und Multikulturalität in der Gesellschaft sowie Gesetzesänderungen zu integrativen und inklusiven Schulformen in der Schweiz 
(Lozano et al., 2017; Reusser et al., 2013) wie auch in Deutschland (Klauß \& Terfloth, 2013; Lütje-Klose, Serke, Hunger \& Wild, 2016; Moser \& Egger, 2017) führen dazu, dass die einzelnen Schülerinnen und Schüler wie auch die Klassenzusammensetzungen zunehmend heterogener werden (Blossfeld et al., 2017; Stebler et al., 2017). Lerngruppen können u. a. hinsichtlich des Alters, des Geschlechts, der sozialen und/oder kulturellen Herkunft, der Schulleistungen oder der Begabungen/Behinderungen heterogen sein (Bohl, Budde \& Rieger-Ladich, 2017; Budde, 2017; Wimmer \& Altrichter, 2017). Im Zuge der Bemühungen um einen für das Lernen gehaltvollen Umgang mit Heterogenität haben sich verschiedene didaktische Konzepte und Ansätze etabliert (u. a. Bohl, Batzel \& Richey, 2011). Zu den prominentesten zählen eine Öffnung des Unterrichts (u. a. Peschel, 2003), innere Differenzierung (u. a. Klafki \& Stöcker, 1976), Individualisierung (u. a. Bräu, 2005) oder adaptiver Unterricht (u. a. Schwarzer \& Steinhagen, 1975). Diese Bestrebungen zielen auf Lernumgebungen ab, in denen Lernbedürfnisse und -voraussetzungen der einzelnen Schülerinnen und Schüler im Vergleich zu einem eng geführten Klassenunterricht stärker gewichtet werden und diese beispielsweise in der Gestaltung der eigenen Lernwege über mehr oder weniger Autonomie- und Handlungsspielräume verfügen (Klieme \& Warwas, 2011; Saalfrank, 2016; Schratz, 2009; Stebler et al., 2018).

\section{Reformpädagogische Bestrebungen eines kindorientierten Unterrichts}

Bereits in Abschnitt 2.3 wurde auf die Bedeutung reformpädagogischer Ansätze aus dem US-amerikanischen Bildungsraum für den englischsprachigen Diskurs über personalisiertes Lernen hingewiesen. Arbeiten beispielsweise von Dewey (1910/2002), der Winnetka-Plan von Washburne (1927) und der Dalton-Plan von Parkhurst (1922) wurden oft rezipiert (u. a. Mincu, 2012a; Yonezawa et al., 2012; Zmuda et al., 2015). Weit weniger zitiert, aber nicht minder bedeutsam sind jedoch europäische Vertreterinnen und Vertreter der klassischen Reformpädagogik, so zum Beispiel Jean-Jacques Rousseau (1712-1778), Rudolf Steiner (1861-1925) und Maria Montessori (1870-1952). Bei Rousseau (1971/1998) heißt es etwa im Buch „Emil oder Über die Erziehung“: „Er weiß nichts von dem, was in der Welt vorgeht, aber er kann das für ihn Richtige tun" (Rousseau, 1971/1998, S. 104). Dies bedeutet, dass ein Kind trotz seiner Unerfahrenheit beim Erkunden der Welt eigene Entscheidungen treffen kann. Auf Lernprozesse übertragen zielt dieser Gedanke auf den Kern personalisierten Lernens ab: Schülerinnen und Schüler verfügen über Autonomie- und Handlungsspielräume, in denen sie selbst Entscheidungen bezüglich ihrer Lernziele, Lerninhalte, Lernorte, Lernmedien und/oder Lernpartnerinnen und Lernpartner treffen können (OECD, 
2006). Lernumgebungen sollen von den Lernenden aus gestaltet werden (lernseits) und können von diesem partizipativ mitbestimmt werden (Schratz, 2017), zum Beispiel bei der Formulierung von Lernzielen: „Learners understand how they learn best so they can become active participants in designing their learning goals along with the teachers“ (Bray \& McClaskey, 2015, S. 11).

Reformpädagogische Unterrichtsmodelle gehen häufig von einer Kritik am öffentlichen Schulsystem aus (u. a. Oelkers, 2005). Standardkonzepte der Vermittlung von schulischem Wissen werden kritisiert und stattdessen Lernumgebungen gefordert, die auf die Lernbedürfnisse und interessen der Kinder ausgerichtet sind. Wichtiger Teil solcher Ansätze sind Lehr- und Lernformen, in denen Schülerinnen und Schüler vermehrt selbstständig und eigenverantwortlich lernen können und sich Lerninhalte sowohl in Einzelarbeit als auch kooperativ mit Mitschülerinnen und Mitschülern aneignen, zum Beispiel im Rahmen von Planarbeit, Werkstattunterricht, Lernateliers oder Projektarbeit. Mit solchen offeneren respektive erweiterten Lehr- und Lernformen geht die Überzeugung einher, dass „die Qualität kognitiver Konstruktionsprozesse durch ein hohes Maß an selbstregulierter Auseinandersetzung mit den Lerninhalten im Sinne des entdeckenden Lernens gefördert wird" (Reusser, 2016, S. 43). Jedoch konnten die „Pythagoras-Videostudie“ (Klieme et al., 2009) und ihre Nachfolgestudie „DidKom“ (Reusser \& Pauli, 2013) aufzeigen, dass weniger die Lehr- und Lernformen und die lernorganisatorischen Merkmale des Unterrichts (Oberflächenstruktur) für die verständnisbezogene Lernqualität bedeutsam sind, sondern vielmehr die Mikrostrukturen des Unterrichts, zum Beispiel fachdidaktisch gehaltvolle, konzeptspezifische, strukturklare und in der Gesprächsführung ko-konstruktive Lernbegleitung (Tiefenstruktur, siehe Abschnitt 4.5.1) (Reusser, 2016; Reusser \& Pauli, 2013).

\section{Potenziale digitaler Medien in individualisierten Lernumgebungen}

Die zunehmende Digitalisierung beeinflusst viele Lebensbereiche und zeigt sich beispielsweise in der Entwicklung der Mobiltelefone: In den 1990er-Jahren wurden diese Geräte vor allem für Telefonate und das Schreiben von Kurznachrichten (SMS) genutzt. Mit der Entwicklung der Smartphones ab den 2000er-Jahren erweiterten sich die Funktionen und die Einsatzmöglichkeiten der Geräte jedoch stark. Im schulischen Kontext können nun zum Beispiel Chaträume eröffnet werden, in denen Lehrpersonen Eltern und/oder Schülerinnen und Schüler kurzfristig über Änderungen im Stundenplan, Ausflüge oder Elternabende informieren.

Auch der Einsatz digitaler Medien im Unterricht hat sich in den letzten Jahren verändert. In den Anfängen waren die Geräte primär Instruktionsmaschinen 
mit dem Ziel, Informationen effizienter zu vermitteln und standardisierte Feedbacks zu geben. Neu sollen die technischen Möglichkeiten kreative und an den Schülerinnen und Schülern orientierte Lehr- und Lernprozesse und die Kommunikation unterstützen (Petko et al., 2017; Reigeluth, 2017). Eine Möglichkeit, die sich durch den Einsatz personalisierter Lernprogramme eröffnet, besteht darin, Daten zum Alter, zur Klassenstufe, zur Schnelligkeit der Beantwortung von Lernaufgaben und zur Richtigkeit der Lösungen etc. zu sammeln, diese auszuwerten und anhand der Ergebnisse passende weitere Lernaufgaben vorzuschlagen, die an das Lernniveau der Schülerinnen und Schüler angepasst sein sollten (Spitzer, 2006; Stebler et al., 2017).

In Anbetracht dessen weisen Informations- und Kommunikationstechnologien (engl. kurz „ICT“) große Potenziale für Unterrichts- und Schulentwicklung in Richtung personalisierten Lernens auf. Studienergebnisse zeigen, dass ICT in Deutschland und in der Schweiz in einem Vergleich mit OECD-Ländern im Durchschnitt eher selten eingesetzt werden (OECD, 2015), in an Schülerinnen und Schülern orientierten Lernumgebungen mit Formen individualisierten Lernens jedoch verstärkt genutzt werden (u. a. Petko, 2012).

\section{Zunehmende Bedeutsamkeit überfachlicher Kompetenzen}

Die steigende Zahl digitaler Lösungen im privaten, gesellschaftlichen und beruflichen Leben ist mit ein Grund, warum sich die Anforderungen der zukünftig berufstätigen Bevölkerung verändern werden: Gleich ablaufende Routinearbeiten wie zum Beispiel Fließbandarbeit werden zunehmend automatisiert. Die Anzahl an Berufstätigkeiten, bei denen komplexe Probleme gelöst und schlecht strukturierende Handlungsabläufe bearbeitet werden, dürfte hingegen markant steigen. Hier werden in meist kooperativen Arbeitsprozessen individuelle Wissens- und Erfahrungsbestände, Fähigkeiten und Fertigkeiten der Mitarbeitenden miteinander verknüpft (u. a. Levy \& Murnane, 2005). Um Schülerinnen und Schüler auf solche anspruchsvollen Tätigkeiten vorzubereiten, werden überfachliche Kompetenzen in zunehmendem Masse bedeutsamer. Zu diesen Kompetenzen wird beispielsweise im Lehrplan 21 Folgendes festgehalten: Schülerinnen und Schüler erwerben

personale, soziale und methodische Fähigkeiten, die für eine erfolgreiche Bewältigung unterschiedlicher Aufgaben in verschiedenen Lebensbereichen zentral sind. Sie lernen, über sich selbst nachzudenken, den Schulalltag und ihr Lernen zunehmend selbstständig zu bewältigen, an der eigenen Lernfähigkeit zu arbeiten, vorgegebene und eigene Ziele und Werte zu verfolgen und zu reflektieren. Sie erwerben soziale und kommunikative Fähigkeiten und lernen, mit anderen Kindern zusammenzuarbeiten, 
Konflikte zu lösen und mit Vielfalt umzugehen. Sie erwerben umfassende sprachliche Kompetenzen, lernen mit Informationen sachgerecht umzugehen und entwickeln Problemlösefähigkeiten. (D-EDK, 2014, S. 4)

Überfachliche Kompetenzen können einerseits im schulischen Zusammenleben, andererseits im Unterricht, etwa bei der vertieften Auseinandersetzung mit fachlichen Lerninhalten, gefördert werden (D-EDK, 2014). Insbesondere verschiedene Formen des problemorientierten Lernens bieten diesbezüglich große Potenziale, weil damit ein kognitiv aktivierender Unterricht gefördert wird, bei dem Schülerinnen und Schüler selbstständig allein oder kooperativ mit Mitschülerinnen und Mitschülern Probleme lösen. Hierbei nimmt die Lehrperson eine Schlüsselrolle bezüglich Qualität und Wirksamkeit ein (u. a. Reusser, 2005).

\section{Rechenschaftsablegung (Accountability)}

Ein weiterer Auslöser von Schulentwicklung, insbesondere auch aus governancetheoretischer Perspektive, ist die Rechenschaftsablegung (Accountability) in Bezug auf schulisches und unterrichtliches Handeln. Politik und Gesellschaft fordern zunehmend, dass unterrichtliche und darüber hinausgehende Tätigkeiten durch etwa externe Evaluationen oder Schulinspektionen anhand von Bildungsstandards überprüft und allfällige Mängel behoben werden (Blossfeld et al., 2017; Carman, 2008; Fend, 2008b; Stebler et al., 2017, 2018).

\section{Sicherung des Schulstandorts}

Ein letzter im uSpL-Modell berücksichtigter Auslöser ist die Sicherung des Schulstandorts. Schulstandorte können - vor allem in ländlichen Regionen mit sinkenden Zahlen an Schülerinnen und Schülern - aufgrund der eingeschränkten Finanzierbarkeit gefährdet sein. In einigen Schulen wird dies zum Anlass genommen, die Jahrgangsklassen aufzulösen und altersdurchmischte Lerngruppen mit vermehrt individualisierenden und/oder binnendifferenzierenden Lehr- und Lernformen einzuführen. Eine solche Entwicklung konnte beispielsweise eine Studie zu Schulen im alpinen Raum nachzeichnen (u. a. Raggl et al., 2015; SommerSutter, 2012). Die Einführung innovativer Unterrichtskonzepte kann in solchen Fällen nicht nur die Finanzierbarkeit von Schulen absichern, sondern darüber hinaus auch deren Profile schärfen und die Attraktivität der Bildungsstandorte erhöhen. 


\subsection{Entwicklungsdimensionen einer unterrichtszentrierten Schulentwicklung in Richtung personalisierten Lernens}

Die in Abschnitt 4.4 beschriebenen Auslöser können in Einzelschulen Entwicklungsprozesse in Richtung personalisierten Lernens bewirken. Im Kern solcher Entwicklungsprozesse werden seit Jahrzehnten tradierte Vorstellungen und Strukturen von Unterricht und Schule überdacht und so verändert, dass sich Schülerinnen und Schüler vermehrt selbstständig und eigenverantwortlich, in Begleitung der Lehrpersonen oder/und mit Mitschülerinnen und Mitschülern zusammen fachliche und überfachliche Kompetenzen aneignen können. Solche Lernumgebungen unterscheiden sich von traditionellen Unterrichtssettings, bei denen eine Gruppe von Schülerinnen und Schülern einer Lehrperson gegenübersitzt, was sich auch in den Klassenzimmern manifestiert. So werden einige Lernräume in einem Schulhaus umstrukturiert und teils umgebaut, sodass Lernorte zur Verfügung stehen, zwischen denen die Schülerinnen und Schüler selbstständig wechseln können und in denen mithilfe unterschiedlicher Regeln verschiedene Lernkulturen geschaffen werden, etwa ruhige Einzelarbeitsplätze mit Flüsterkultur oder Gruppenräume, in denen die Schülerinnen und Schüler kooperativ Lernaufgaben lösen.

Doch nicht nur die Lernräume, sondern auch darüber hinausgehende Bereiche des Unterrichts und der Schule werden verändert: Lerngruppen werden heterogener zusammengesetzt, die Lerninhalte werden je nach Konstellation der Lerngruppenstruktur neu angeordnet und in Wahl- und Pflichtinhalte differenziert, die Lernbegleitung wird in Richtung einer intensivierten individuellen Lernunterstützung weiterentwickelt etc. (u. a. Müller, 2014; Müller, 2015; Zylka, 2017). Die Anzahl der möglichen Entwicklungstätigkeiten ist groß. Um diese Vielfalt zu systematisieren, wurden Entwicklungsdimensionen definiert, die dem uSpL-Modell (siehe Abschnitt 4.2) und der Datenanalyse in Studie 1 (siehe Abschnitt 6.4.1) zugrunde liegen. Unter „Entwicklungsdimensionen“ sind in diesem Zusammenhang auf der theoretischen Literatur aufbauende Kategorisierungen von Entwicklungstätigkeiten zu verstehen.

In der deutschsprachigen Literatur lassen sich verschiedene Kategorisierungen finden. Klafki (1985/2007) unterschied bereits 1985 in seiner zehnten Studie zu Thesen der inneren Schulreform zwei Hauptfelder: (1) Unterricht und (2) das über den Unterricht hinausreichende Schulleben (Feiern, Pausengestaltung, Schulhofgestaltung etc.). Im häufig rezipierten Drei-Wege-Modell von Rolff (1998, 2013) werden demgegenüber drei Entwicklungsdimensionen beschrieben: Personal-, Organisations- und Unterrichtsentwicklung. Zur Personalentwicklung zählt Rolff die Kompetenzentwicklung des Schulpersonals und fasst darunter u. a. die 
folgenden Tätigkeiten: Lehrpersonenfeedback, Supervision und Coaching, Kommunikationstraining, Schulleitungsberatung, Hospitation, Jahresgespräche und Zielvereinbarungen sowie Führungsfeedback. Organisationsentwicklung besteht gemäß Rolff (2013) in der Entwicklung von Organisationsklima, -strukturen und -abläufen, die eine Schule zu einer lernenden Organisation formt. Dazu gehören Aspekte wie Schulprogramm, Schulkultur, Erziehungsklima, Schulmanagement, Teamentwicklung, Evaluation, Kooperation oder Steuergruppe. Die letzte Entwicklungsdimension ist die Unterrichtsentwicklung, unter der die didaktische Weiterentwicklung des Unterrichts zusammengefasst wird und die sich u. a. auf Fachlernen, Orientierung an den Schülerinnen und Schülern, überfachliches Lernen, Methodentraining, Selbstlernfähigkeit, Öffnung, erweiterte Unterrichtsform und Lernkultur bezieht.

Während Rolff die Entwicklungsdimensionen im Drei-Wege-Modell nicht hierarchisiert, wurde dies in einem Modell inklusiver Schulentwicklung von Lozano et al. (2017) vorgenommen. Hier ist die Unterrichtsentwicklung zentral in der Mitte positioniert. Um den Unterricht sind die Einstellungen aller Beteiligten zu einer inklusiven Schule und einem inklusiven Unterricht, Schule und Leitung, die professionelle Kooperation in multiprofessionellen Teams, die Kooperation mit den Eltern, Tagesstrukturen, die schulergänzende Kooperation sowie professionelle Handlungskompetenzen angeordnet. Umrahmt werden diese Dimensionen von den rechtlichen Grundlagen.

Unter Berücksichtigung der einschlägigen Literatur insbesondere zu personalisiertem Lernen (u. a. Bray \& McClaskey, 2015; Galle et al., 2019; D. Hargreaves, 2006a; Mincu, 2012a; Petko et al., 2017; Prain et al., 2015a; Sebba et al., 2007; Stebler et al., eingereicht; Stebler et al., 2018; Waldrip et al., 2016) lassen sich im Kontext einer unterrichtszentrierten Schulentwicklung in Richtung personalisierten Lernens im Sinne einer Synthese fünf Entwicklungsdimensionen ableiten (Abbildung 4.4): Unterrichtsentwicklung in Richtung einer personalisierten Gestaltung von Lehr- und Lernprozessen (Abschnitt 4.5.1), Entwicklung organisationaler Strukturen (Abschnitt 4.5.2), Entwicklung der Kooperation zwischen Lehrpersonen (Abschnitt 4.5.3), Professionalisierung des Schulpersonals und Personalrekrutierung (Abschnitt 4.5.4) sowie Entwicklung einer (SchulGemeinschaft (Abschnitt 4.5.5).

Die Entwicklungsdimensionen im uSpL-Modell (Abbildung 4.4) bedingen sich gegenseitig und stehen unter einem wechselseitigen Einfluss. Wenn zum Beispiel Jahrgangsklassen zu alters- und/oder leistungsdurchmischten Lerngruppen zusammengelegt und in Phasen offenen Unterrichts in Lernlandschaften unterrichtet werden, dann braucht es für diesen Unterricht aufbereitete Lernmaterialien und Stoffpläne mit Wahlmöglichkeiten (u. a. Müller, 2014, 2015). Die dafür 


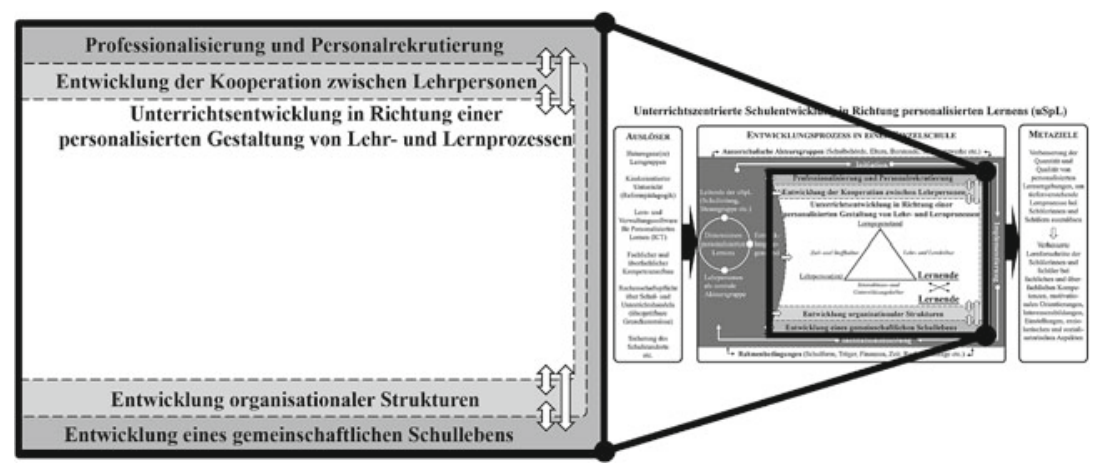

Abbildung 4.4 Entwicklungsdimensionen im uSpL-Modell (eigene Darstellung)

nötigen Unterrichtsvorbereitungen kann meist nicht mehr eine Lehrperson allein vollbringen, vor allem dann nicht, wenn auf Sekundarstufe die verschiedenen Fachexpertisen eine zentrale Rolle spielen. Hier ist eine intensive Kooperation zwischen Lehrpersonen gefordert, die Unterricht miteinander und füreinander vorbereiten (u. a. Galle et al., 2019; Stebler et al., eingereicht). Eine solche intensive, meist ko-konstruktive Kooperation beinhaltet aufgrund des gegenseitigen Aufeinanderbeziehens von Wissens- und Erfahrungsbeständen Potenziale für nachhaltige Professionalisierungsprozesse (Gräsel, Fussangel \& Pröbstel, 2006a; Keller-Schneider \& Albisser, 2012; Maag Merki, Kunz, Werner \& Luder, 2010).

Des Weiteren sind die Entwicklungsdimensionen hierarchisch angeordnet: Im Zentrum steht die Unterrichtsentwicklung. Darum herum sind die Entwicklung der Kooperation zwischen Lehrpersonen sowie die Entwicklung organisationaler Strukturen positioniert. Im dritten Kreis verortet sind zum einen Professionalisierung und Personalrekrutierung und zum anderen die Entwicklung eines gemeinschaftlichen Schullebens. Diese Anordnung beruht einerseits auf der bereits oben beschriebenen Zentralität von Unterrichtsentwicklung, weil Entwicklungstätigkeiten auf die Lernebene abzielen sollen, um den Lernerfolg der Schülerinnen und Schüler zu verbessern (u. a. Maag Merki, 2017; Reusser, 2011). Andererseits bildet dies auch die empirischen Ergebnisse von Studie 1 ab (siehe Abschnitt 7.1). Da das Modell in einem deduktiv-induktiven Arbeitsprozess (siehe Abschnitt 4.1) konzipiert wurde, ist die Hierarchisierung auch Abbild der durch die Leitfäden gesetzten Gesprächsimpulse zum einen und der von den interviewten Personen berichteten Entwicklungstätigkeiten zum anderen. Konkret bedeutet dies, dass zur Entwicklung des Unterrichts in Richtung einer personalisierten Gestaltung von 
Lehr- und Lernprozessen die umfangreichsten Leitfragen dazu gestellt wurden und auch am meisten und am differenziertesten berichtet wurde. Im Gegensatz dazu wurden zur Professionalisierung und Personalrekrutierung sowie zur Entwicklung eines gemeinschaftlichen Schullebens die wenigsten Fragen gestellt und es wurde in den Interviews am wenigsten darüber berichtet.

\subsubsection{Unterrichtsentwicklung in Richtung einer personalisierten Gestaltung von Lehr- und Lernprozessen}

Wie bereits ausgeführt, ist die Weiterentwicklung des Unterrichts zentral. Mit Fokus auf personalisiertes Lernen (siehe Kapitel 1) zielen Entwicklungstätigkeiten auf die Ermöglichung und die Gestaltung personalisierter Lernprozesse ab. Während das Verständnis der Personalisierung von Lernprozessen mit fünf Dimensionen bereits dargelegt wurde (siehe Abschnitt 3.2), stellt sich nachfolgend die Frage, wie wirkungsvolle Lernprozesse, in denen sich Schülerinnen und Schüler aktiv mit Lerngegenständen auseinandersetzen und diese tiefgehend durchdringen, charakterisiert werden können (Abschnitt 4.5.1.1). Anschließend wird den Fragen nachgegangen, wie Unterricht auf der Grundlage eines solchen Lernverständnisses und aus einer allgemeindidaktischen Perspektive definiert werden kann (Abschnitt 4.5.1.2) und auf welche Unterrichtsaspekte Entwicklungstätigkeiten abzielen sollten, um wirkungsvolle Lernprozesse zu evozieren (Abschnitt 4.5.1.3). Im letzten Unterkapitel werden empirische Ergebnisse zu Entwicklungstätigkeiten in Richtung personalisierter Lernkonzepte zusammengestellt (Abschnitt 4.5.1.4).

\subsubsection{Lernprozesse aus kognitiv- und soziokonstruktivistischer Perspektive}

Durch Schul- und Unterrichtsentwicklung sollen Lernumgebungen so verändert werden, dass Schülerinnen und Schüler nachhaltiger und wirkungsvoller lernen können (u. a. Maag Merki, 2017). Dieser Anspruch wird insbesondere in der Literatur zur Schulentwicklung häufig ohne Klärung des zugrunde liegenden Lernverständnisses vertreten. Ausnahme bilden nach bisherigem Recherchestand das Modell Deep Learning von Fullan, Quinn und McEachen (2018). Die Forschungsgruppe vertritt die These, dass Entwicklungstätigkeiten an Merkmalen einer qualitätsvollen Lernumgebung ausgerichtet werden sollten, in der Schülerinnen und Schüler aktiv, problemorientiert und verstehensbezogen lernen können 
und in ihren kognitiven Aktivitäten herausgefordert werden. Um Entwicklungsprozesse theoretisch zu fassen, ist das Verständnis von Lernen und Unterricht zentral. Auf dieser Grundlage wird es möglich, die Unterrichtsmerkmale zu eruieren, die hohe Effekte auf den Lernerfolg von Schülerinnen und Schülern aufweisen. Das uSpL-Modell gründet auf einem kognitiv- und soziokonstruktivistischen Lernverständnis.

\section{Kognitiv-konstruktivistisches Lernverständnis}

Kognitiv-konstruktivistisch ist ein kognitiver Aufbau von Wissens- und Denkstrukturen, bei dem neues Wissen an bereits vorhandene Wissens- und Denkstrukturen angeknüpft wird (u. a. Hasselhorn \& Gold, 2006; Reich, 2008). Für eine erfolgreiche Verknüpfung ist es bedeutsam, dass bereits vorhandenes Wissen beweglich (reversibel) und leicht zugänglich ist und das zu erwerbende Wissen leicht daran angeschlossen werden kann (Reusser, 2019). Dies bildet eine Grundlage für intensive und tiefgehende Auseinandersetzungen mit Lerninhalten. Des Weiteren ist für einen Lernprozess Folgendes zentral:

Je aktiver, selbstständiger, problem- und handlungsorientierter neues Wissen erworben, durchgearbeitet und konsolidiert wird, desto besser wird es verstanden (Transparenz, Klarheit), [desto] dauerhafter wird es behalten (Stabilität), [desto] beweglicher kann es beim Denken und Handeln in neuen Kontexten genutzt werden (Transfer, Mobilität), [desto] höher sind die Erträge hinsichtlich Aufbau und Mitübung von Lernkompetenzen [und desto] positiver werden die damit verbundenen Lernprozesse erlebt (Motivationsgewinn, Interesse, Relevanz, Selbstwirksamkeit). (Reusser, 2016, S. 41)

Ein solches Lernverständnis lässt sich in unterschiedlichen Differenzierungsgraden in Konzeptionen personalisierten Lernens wiederfinden (u. a. Bray \& McClaskey, 2015; Zmuda et al., 2015). Besonders deutlich wird dies, wenn Autorinnen und Autoren reformpädagogische Ideen rezipieren (siehe Abschnitt 2.3), die sich darin manifestieren, dass beispielsweise kognitive Konstruktionsprozesse gefördert werden sollten, indem Lernende ihre Lernprozesse in einem hohen Masse selbst regulieren können. Schülerinnen und Schüler können vermehrt Lerninhalte gemäß ihren Interessen auswählen und sie sich in Phasen des selbstständigen und eigenverantwortlichen Lernens aneignen. Für die Nutzung der Freiräume sind u. a. Strategien erforderlich, mit denen Schülerinnen und Schüler ihr Lernverhalten hinsichtlich einer tiefgehenden Auseinandersetzung mit Lerninhalten regulieren können. Solche Lernstrategien können die Schülerinnen und Schüler durch angeleitetes Üben und Kultivieren in fachlich anspruchsvollen Situationen erlernen (Reusser, 2019). 
Die Aneignung von Lerninhalten oder -strategien kann aus kognitivkonstruktivistischer Perspektive nicht verordnet werden und muss von jeder einzelnen Schülerin und jedem einzelnen Schüler selbst vollzogen werden (u. a. Lipowsky, 2009; Siebert, 2005). Zudem ist eine solche kognitive Konstruktion zum großen Teil eine Nachkonstruktion von bereits vorhandenen Wissens- und Denkstrukturen, die jedoch auf selbst gewählten Wegen eigenständig (nach)vollzogen werden (Reusser, 2019). Dabei können Irritationen, Widerstände und Verstehensklippen auftreten, die kognitive Konflikte auslösen und für deren Auflösung bisherige Wissens- und Denkstrukturen reorganisiert und akkommodiert werden müssen (Oser \& Spychiger, 2005; Reusser, 2006, 2016).

\section{Soziokonstruktivistisches Lernverständnis}

Lernen ist nicht nur ein kognitiver, sondern auch ein sozialer Prozess. Komplexe Wissensstrukturen und tragfähige Lern- und Denkfähigkeiten werden interaktiv und durch die Partizipation in Wissensbildungsgemeinschaften erworben (Stebler, Reusser \& Pauli, 1994). Angelehnt am sozialen Konstruktivismus (Vygotskij, 1934/2002) wird angenommen, dass individuelle Wissenskonstruktionen in einen sozialen Kontext eingebettet sind und von diesem beeinflusst werden. Im Unterricht sind Lernprozesse in einer Lerngemeinschaft situiert und können durch das kooperative Handeln der Lehrpersonen und der Mitschülerinnen und Mitschüler unterstützt oder gehemmt werden. Förderlich sind diesbezüglich u. a. ko-konstruktive Dialoge, wechselseitiges Feedback, Feedup und Feedforward (Reusser, 2016; Woolfolk, 2008). Um die Konstruktion von Wissens- und Denkstrukturen im sozialen Kontext zu ermöglichen, sind zudem Denkwerkzeuge und Symbolsysteme wie zum Beispiel Schrift, Sprache, Zahlensystem oder die oben bereits erwähnten Lernstrategien und deren Kultivierung notwendig (Reusser, 2016).

\subsubsection{Didaktische Unterrichtsgestaltung aus kognitiv- und soziokonstruktivistischer Perspektive}

Unterricht ist das Kerngeschäft von Lehrpersonen und in einer Schulentwicklung, die auf verbesserten Lernerfolg von Schülerinnen und Schülern abzielt, zentral. Unterricht besteht im Kern in der Vermittlung und der Aneignung von kulturbedeutsamen fachlichen und überfachlichen Inhalten und Kompetenzen:

In systematischer Sicht handelt es sich um einen sozialen und gegenstandsbezogenen Prozess des Wissens- und Kompetenzaufbaus, bei dem Personen in komplementären Rollen - berufsmäßig Lehrende sowie Gruppen von Lernenden - bezogen auf gegebene 
Ziele und im Kontext von Normen und Rahmenbedingungen sich in planvoller Weise mit Sach- und Kulturgegenständen auseinandersetzen. (Reusser, 2009, S. 881)

Eine bereits etwas ältere Denkfigur zur Abbildung der Grundstruktur von Unterricht ist das in Abschnitt 4.2 bereits erwähnte didaktische Dreieck (Abbildung 4.5; u. a. Reusser, 2008) mit den Ecken „Stoff/Gegenstand“, „Lernende“ und „Lehrperson“. Die Kanten zwischen den Ecken konstituieren drei didaktische Teilkulturen, die zugleich Kernaufgaben von Lehrpersonen darstellen: die bildungsinhaltliche Ziel- und Stoffkultur (Was soll warum und wozu gelehrt werden?), die auf den Lehr- und Lernprozess bezogene Lehr- und Lernkultur (Wie und in welcher Prozessqualität soll gelernt und gelehrt werden?) und die kommunikativunterstützende Interaktions- und Unterstützungskultur (Womit und wodurch kann Lernen unterstützt, begleitet und ermutigt werden?). Kernzone didaktischen Handelns der Lehrperson sind entlang der drei Teilkulturen die inhalts-, prozessund interaktionsbezogenen Aufgaben sowie die Steuerung der kognitiven, sozialen und motivational-emotionalen Dynamiken im Lehr- und Lerngeschehen in Abhängigkeit von den Lernenden und den Lerngegenständen.

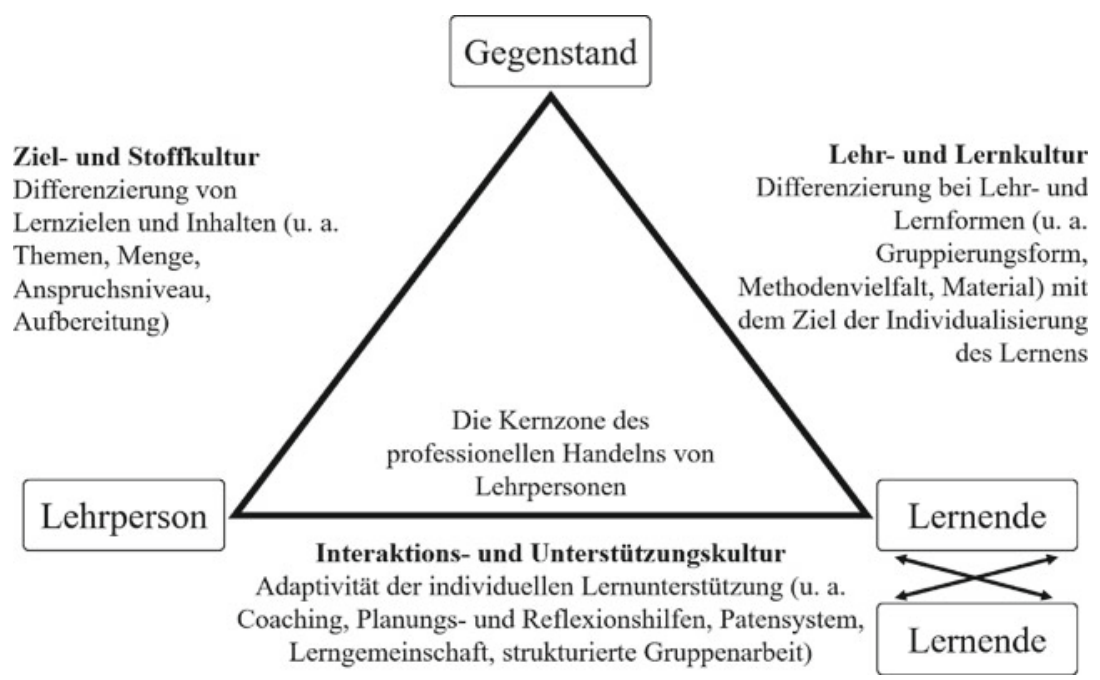

Abbildung 4.5 Didaktisches Dreieck mit drei Teilkulturen didaktischen Handelns (Reusser, 2008) 


\section{Ziel- und Stoffkultur}

Zentrale Aufgabe im Unterricht ist die Vermittlung von relevanten Bildungsinhalten. Im öffentlichen Bildungssystem werden diese vor allem von verantwortlichen Personen der Systemebene definiert und liegen den Lehrpersonen in Form von Lehrplänen vor. In den letzten Jahren wurde und wird intensiv über Bildungsstandards diskutiert und diese werden zunehmend im Schulsystem implementiert (Criblez et al., 2009; Klieme et al., 2003; Oelkers \& Reusser, 2008). Auch die stärkere Gewichtung von überfachlichen Kompetenzen, zum Beispiel im Lehrplan 21 (D-EDK, 2014), ist Zeichen für sich stetig verändernde Ansprüche und Vorstellungen von Bildungs- und Sozialisationszielen sowie von Lerninhalten in Schule und Unterricht. Lehrpersonen stehen vor diesem Hintergrund vor der Herausforderung, die Lerninhalte in kognitiv aktivierenden Aufgaben zu integrieren, sie in Formen eines dialogischen, problemlösend-entdeckenden Lernens in bewältigbare Denk- und Wissensstrukturen zu unterteilen und dabei die ursprünglichen, den Lerninhalten zugrunde liegenden Denkprozesse und Entdeckungszusammenhänge freizulegen, damit die Schülerinnen und Schüler diese mit eigenen Denkund Wissensstrukturen verknüpfen können (u. a. Reusser, 2019).

\section{Lehr- und Lernkultur}

Um Bildungsinhalte vermitteln zu können, braucht es entsprechende Lernumgebungen. Lehrpersonen gestalten diese durch verschiedene Methoden sowie Handlungs-, Sozial- und Unterrichtsformen. Zudem sind Lehrpersonen für die tiefer gehenden, psychologischen Prozessqualitäten verantwortlich. Lernprozesse können einerseits dann als qualitätsvoll bezeichnet werden, wenn sich Schülerinnen und Schüler in intensiven kognitiven Verarbeitungsprozessen mit Wissen auseinandersetzen, dieses tief durchdringen und die dahinterliegenden Wissensund Denkstrukturen verstehen und diese in andere Kontexte transferieren können. Andererseits müssen Schülerinnen und Schüler für verstehensbezogenes Lernen qualifiziert werden, indem sie Arbeitsmethoden und Lernstrategien durch angeleitetes Üben und Kultivieren in fachlich anspruchsvollen Situationen erwerben (u. a. Reusser, 2019).

\section{Interaktions- und Unterstützungskultur}

Die Interaktions- und Unterstützungskultur gründet auf der Erkenntnis, dass Bildungswirkungen nicht nur durch das alleinige Beschäftigen mit Sach- und Kulturgegenständen erzielt werden können, sondern auch in der interpersonalen Auseinandersetzung mit diesen. Die Gestaltung einer möglichst fruchtbaren Lehr- und Lernbeziehung, in der Schülerinnen und Schüler kognitiv, sozial und 
emotional unterstützt werden, kann für positive Lerneinstellungen, Selbstwirksamkeitserleben sowie Vertrauen in die eigenen Fähigkeiten und Fertigkeiten wichtig sein. Bedeutsam ist in der Gestaltung der persönlichen Interaktion, dass auf die individuellen Eigenschaften und Bedürfnisse der einzelnen Lernenden eingegangen wird. So braucht beispielsweise jede Schülerin und jeder Schüler eine eigene lernunterstützende Kommunikation, um etwa fachliche Erfolge und Misserfolge zu besprechen, Krisen zu lösen, Grenzen zu setzen oder Fehler zu korrigieren (u. a. Reusser, 2019).

Didaktisches Handeln in der personalisierten Gestaltung von Lehr- und Lernprozessen entlang der drei Teilkulturen des didaktischen Dreiecks

Zum Abschluss dieses Unterkapitels werden anhand des didaktischen Dreiecks Merkmale und Charakteristika didaktischen Handelns in personalisierten Lernumgebungen expliziert, die sich entlang der fünf Dimensionen personalisierten Lernens beschreiben lassen (siehe Abschnitt 3.2). Diese Ausführungen enden in einer leichten Anpassung des didaktischen Dreiecks (Abbildung 4.6).

Der Kern didaktischen Handelns ist in der personalisierten Gestaltung von Lehr- und Lernprozessen derselbe wie in anderen didaktischen Ansätzen und Konzepten: „Ziel des didaktischen Handelns von Lehrpersonen ist es, Schülerinnen und Schülern bei der Auseinandersetzung mit der dinglichen und sozialen Welt und im Umgang mit kulturellen Werkzeugen und Symbolsystemen Gelegenheit zu einem handlungs- und problemorientierten, verständnisvollen und dialogischen Lernen zu geben“" (Reusser, 2008, S. 227). Jedoch zeigt sich, dass durch Formen der Individualisierung, der inneren Differenzierung und der Öffnung des Unterrichts die Komplexität didaktischen Handelns im Vergleich zu beispielsweise einem eng geführten Klassenunterricht ansteigt. Dies zeigt sich etwa in der Auf- und Vorbereitung von Lerninhalten. Die Anpassung von Lerninhalten und Bildungszielen an die personalen Voraussetzungen von einzelnen Schülerinnen und Schülern oder Lerngruppen bedingt eine differenzierte Unterrichtsvorbereitung und -planung für verschiedene Leistungsniveaus. Dies ist besonders dann herausfordernd, wenn die Lerngruppen alters- und/oder leistungsdurchmischt zusammengesetzt werden (u. a. Achermann \& Gehrig, 2013). Lerninhalte können zum Beispiel so aufbereitet werden, dass Schülerinnen und Schüler aus Pflichtund Wahlbereichen wählen können. Diese Wahlmöglichkeiten eröffnen ihnen ein Lernen auf eigenem Weg (u. a. Müller, 2014).

Beim Lernen auf eigenem Weg steuern Schülerinnen und Schüler ihre Lernprozesse vermehrt selbst, jedoch im von der Lehrperson definierten Rahmen. Das Lernen auf eigenem Weg wird auch durch eine differenzierte Lernarchitektur unterstützt. So werden beispielsweise Räume mit verschiedenen Lernkulturen 
gestaltet oder ein Raum wird in verschiedene Lernzonen eingeteilt. Auf diese Weise lassen sich ruhige Lernorte für Einzelarbeiten, Orte mit Flüsterkultur für Partner- und Gruppenarbeiten oder in Primarschulen auch Lernorte mit Spielmöglichkeiten einrichten (Bachmann, 2015a, 2015b). Um selbstreguliert lernen zu können, sind Schülerinnen und Schüler aufgefordert, sich u. a. überfachliche Kompetenzen anzueignen, wie etwa sich selbst zu motivieren, Arbeiten einzuteilen, eigene Lernziele selbstständig zu verfolgen oder Lernprozesse zu reflektieren. Damit übernehmen sie Eigenverantwortung für ihr Lernen. Mit der Eigenverantwortung und dem Zusprechen von Handlungs- und Autonomiespielräumen zielen personalisierte Lernumgebungen auch auf eine ganzheitliche Förderung der Schülerinnen und Schüler ab und fördern neben fachlichen und überfachlichen Kompetenzen auch personale und soziale Kompetenzen.

Allerdings bedeuten die ausgeprägten Autonomieräume nicht, dass die Schülerinnen und Schüler allein gelassen werden. In solch offeneren und teils individualisierten Lernsettings ist vielmehr eine adaptive, individuell angepasste Lernunterstützung notwendig (Reusser, 2016). Insbesondere bei leistungs- und konzentrationsschwachen Schülerinnen und Schülern, die größere Schwierigkeiten mit dem selbstständigen Aufgabenlösen, der Organisation von Lernprozessen sowie der Impulskontrolle und Selbstdisziplin haben können, braucht es eine vergleichsweise enge und intensivierte Lernunterstützung (Lipowsky, 2002; Lipowsky \& Lotz, 2015). Im Zusammenhang mit Personalisierung werden auch Formen des individuellen Coachings als Form der Lernunterstützung beschrieben, die teils in regelmäßigen Einzelgesprächen stattfindet und strukturell fest im Unterrichtsund Schulbetrieb verankert ist (Müller, 2014, 2015).

Diese exemplarischen Ausführungen geben einen Einblick in die Komplexität didaktischen Handelns in personalisierten Lernkonzepten. Um diese Komplexität, u. a. hervorgerufen durch die zunehmende Heterogenität von Lerngruppen, im Berufsalltag bewältigen zu können, arbeiten Lehrpersonen zunehmend in Teams zusammen: Beispielsweise bereiten sie miteinander oder füreinander Unterricht vor, setzen verschiedene Formen des Team-Teachings ein oder/und reflektieren gemeinsam ihren Unterricht (u. a. Galle et al., 2019; Hildebrandt, Ruess, Stommel \& Brühlmann, 2017; Kreis, Wick \& Kosorok Labhart, 2016; Reusser et al., 2013; Stebler et al., eingereicht). Die unterrichtsbezogene Kooperation in Teams kann sich auf das didaktische Handeln auswirken, weil dieses nicht nur auf die individuellen Ansprüche und Bedürfnisse der Schülerinnen und Schüler abgestimmt werden sollte, sondern auch ein sinnvolles Ineinandergreifen und Ergänzen mit dem didaktischen Handeln anderer Lehrpersonen erfordert. Deswegen gilt es, das didaktische Dreieck leicht anzupassen, indem der Begriff „Lehrperson“ mit der Endung ,,-(en)“ ergänzt wird (Abbildung 4.6). 


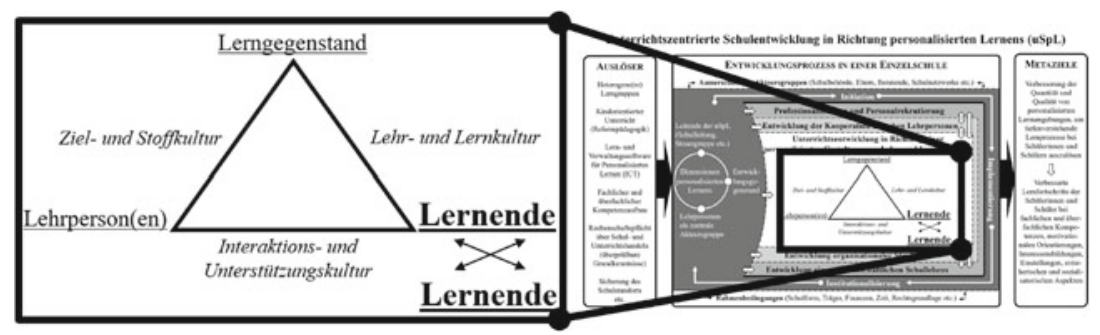

Abbildung 4.6 Angepasstes didaktisches Dreieck (Reusser, 2008) im uSpL-Modell (eigene Darstellung)

Zudem wurde der Begriff „Lernende“ in der adaptierten Version des Dreiecks fett formatiert und grösser geschrieben als die Begriffe „Lehrperson(en)“ und „Lerngegenstand“, weil mit personalisierten Lernumgebungen der Anspruch verbunden ist, den Unterricht ausgehend von den Schülerinnen und Schülern das heißt lernseits - zu planen und umzusetzen (u. a. Bray \& McClaskey, 2015; Schratz, 2017).

\subsubsection{Nachhaltige Entwicklung personalisierter Lernkonzepte: Oberflächen- und Tiefenmerkmale lernwirksamen Unterrichts als zentrale Bezugspunkte von Entwicklungstätigkeiten}

Eine den Ausführungen im vorhergehenden Kapitel entsprechende Zentrierung der Schülerinnen und Schüler und ihrer Lernprozesse ist im Verständnis des uSpLModells sowohl Ausgangs- als auch Zielpunkt von Entwicklungstätigkeiten (siehe Abschnitt 4.2). Deswegen steht ein selbstständiges Lernen fördernder und kognitiv aktivierender Unterricht im Zentrum. In Theorie und Forschung zu qualitätsvollem Unterricht wird zwischen Oberflächenstruktur und Tiefenstruktur unterschieden (Klieme et al., 2008; Kunter \& Trautwein, 2013; Lipowsky et al., 2009; Reusser \& Pauli, 2013; Reusser et al., 2010). ${ }^{1}$ Die Differenzierung geht zurück auf Aebli (1961, 1983/2006) und wurde in Arbeiten von Oser (Oser \& Baeriswyl, 2001; Oser \& Patry, 1990) sowie Kurt Reusser (1999) und seitdem in zahlreichen weiteren Arbeiten aufgegriffen. In Anlehnung an Aeblis psychologische Didaktik lassen sich auf der Oberflächen-, Sicht- oder Handlungsstrukturebene die dramaturgischen Gestaltung und die Choreografien des Unterrichts sowie das variable

\footnotetext{
${ }^{1}$ Eine kritische Reflexion der beiden Begriffe Oberflächen- und Tiefenmerkmale haben Decristan, Hess, Holzberger und Praetorius (2020) vorgelegt.
} 
Spiel aus Methoden, Sozial- und Inszenierungsformen lokalisieren, während die Tiefenstruktur von Unterricht das ,erkenntnispsychologische Aktgefüge des Lernens und dessen prozessbezogene, psychologisch-didaktische Tiefenqualitäten“ (Reusser, 2019, S. 144 f.) umfasst. Tiefenmerkmale beziehen sich auf psychologische Prozesse und Merkmale des Lehrens und Lernens, die dem Unterricht als psychologisch-didaktische Qualitätsmerkmale zugrunde liegen (Reusser \& Pauli, 2010b). Beim didaktischen Handeln geht es diesbezüglich etwa um die Frage, ob die Schülerinnen und Schüler mitdenken, sich aktiv beteiligen und die Umsetzung des dramaturgischen Aufbaus mittragen. Des Weiteren wird danach gefragt, wie es die Lehrperson schafft, den Schülerinnen und Schülern die Lerninhalte aktivierend näherzubringen und ihr Interesse zu wecken, sodass sie sich intensiv damit auseinandersetzen, die dahinterliegenden Wissens- und Denkstrukturen erfassen und verstehen und diese mit eigenen Wissens- und Denkstrukturen verknüpfen. Der Prozess des Verstehens ist dabei ein wichtiger Bezugspunkt, der im unterrichtlichen Handeln etwa durch geistiges Operieren, durch Problemlösen und durch gedanklichen Strukturaufbau ausgelöst werden kann (Aebli, 1983).

Empirische Studien belegen, dass didaktisches Handeln, welches nicht nur die methodische Oberflächenstruktur und Inszenierungsformen mitdenkt, sondern insbesondere die psychologischen Tiefenqualitäten von Lehr- und Lernprozessen fokussiert, hohe Wirksamkeit auf nachhaltigen Lernerfolg aufweist (Klieme et al., 2008; Lipowsky et al., 2009; Reusser \& Pauli, 2013; Reusser et al., 2010). Auch die Ergebnisse der Meta-Metaanalyse von John Hattie (2009, 2012) unterstreichen - unter Berücksichtigung der methodischen Schwächen (Übertragbarkeit auf deutschsprachigen Bildungsraum, Aktualität der Einzelstudien etc.) - die Bedeutsamkeit solcher Prozessqualitäten didaktischen Handelns (Meyer, 2015; Reusser, 2016). Weit weniger empirisch geklärt sind hingegen das Verhältnis und das Zusammenwirken zwischen unterrichtsmethodischen Inszenierungen und Arrangements und Lernprozessqualität. Auf deskriptiver Ebene stehen die zwei Perspektiven weder in einem additiven noch in einem chronologisch aufeinander aufbauenden Verhältnis zueinander. Es ist vielmehr ein vielfältiges und sich wechselseitig bedingendes Zusammenspiel in komplexen sozialen Unterrichtssituationen (Reusser, 2009; Reusser et al., 2013).

In der vorliegenden Arbeit wird für die Beantwortung der Teilfrage 2.3 bis 2.7 (Studie 2) angenommen, dass in einer unterrichtszentrierten Schulentwicklung die Unterrichtskonzepte in Richtung personalisierten Lernens vor allem dann nachhaltig weiterentwickelt werden können, wenn Entwicklungstätigkeiten nicht nur auf die organisatorischen und strukturellen Merkmale der Oberflächenstruktur 
abzielen, sondern auch die Prozessqualitäten didaktischen Handelns fokussieren (u. a. Reusser, 2011). Deshalb werden Merkmale der Oberflächen- und der Tiefenstruktur des Unterrichts im Folgenden noch ausführlicher dargestellt.

\section{Merkmale der Oberflächenstruktur des Unterrichts}

Die Merkmale der Oberflächenstruktur des Unterrichts beziehen sich wie bereits dargelegt auf lernorganisatorische Handlungen und unterrichtliche Strukturen; sie sind für Außenstehende in relativ kurzer Zeit sichtbar und beobachtbar (u. a. Kunter \& Trautwein, 2013; Reusser, 2008). Als Beispiele hierfür lassen sich Lehr- und Lernformen wie frontaler Lehrpersonenvortrag, Werkstattunterricht, Wochenplan oder Projektunterricht sowie die Unterrichtsmedien nennen. Zu Letzteren gehören einerseits die ,gedruckten“ Medien, beispielsweise in Form von Lehrmitteln und Lernaufgaben, die in Schulen mit Konzepten personalisierten Lernens u. a. in Aufgabensammlungen häufig neu strukturiert werden und zwischen verschiedenen Altersstufen oder/und Leistungsniveaus differenzieren (Müller, 2014). Andererseits sind hierunter digitale Medien (ICT) zu zählen, die bereits als Auslöser für eine Entwicklung in Richtung personalisierten Lernens beschrieben wurden (siehe Abschnitt 4.4). Die Funktionen von Computerprogrammen sind vielfältig: So können Lernprogramme eingesetzt werden, mit denen die Schülerinnen und Schüler selbstständig an Lernaufgaben arbeiten können und die je nach richtigen und falschen Antworten neue, dem Leistungsniveau entsprechende Lernaufgaben zusammenstellen. Ebenso gibt es Programme, um die individuellen Lernprozesse zu dokumentieren und zu verwalten (D. Hargreaves, 2006a; Petko et al., 2017). Ein konkretes Beispiel hierfür ist das Programm „InfoMentor“, 2 das u. a. Schulen des Schulnetzwerks „mosaik-Sekundarschulen“ nutzen. Mit diesem Tool können Lehrpersonen die Lernumgebung organisieren und individuelle Lernprozesse überblicken. So ist es mithilfe des Tools beispielsweise möglich, die bearbeiteten und zukünftig zu lösenden Lernaufgaben sowie die zu erlernenden und bereits erworbenen Kompetenzen für jede Schülerin und jeden Schüler zu dokumentieren.

Ein weiteres Merkmal der Oberflächenstruktur sind Lehrpläne, die vorgeben, welche Lerninhalte eine Schülerin oder ein Schüler wann erarbeiten soll. Lehrpersonen haben diesbezüglich die Aufgabe, diese Lerninhalte in kleinere, für Schülerinnen und Schüler bearbeitbare Einheiten zu unterteilen. In Schulen mit alters- und/oder leistungsdurchmischten Lerngruppen werden beispielsweise Stoffpläne erstellt, in denen die Lerninhalte je nach Zusammensetzung der Lerngruppe neu angeordnet werden (D. Hargreaves, 2006a; Prain et al., 2015a). Zum Beispiel können die Lerninhalte für den Unterricht in altersdurchmischten

${ }^{2}$ Quelle: https://www.infomentor.ch/ [letzter Zugriff: 01.01.2021]. 
Lerngruppen so angeordnet werden, dass Schülerinnen und Schüler mehrerer Jahrgänge an einem gemeinsamen Lernthema arbeiten und in bestimmten Phasen, zum Beispiel beim Üben, in verschiedene Leistungsniveaus differenziert wird (u. a. Achermann \& Gehrig, 2013). Hier schließen die Kompetenzraster an, die darauf abzielen, fachliche und überfachliche Kompetenzziele für jede Schülerin und jeden Schüler zu definieren und deren Erreichung mehr oder weniger überprüfbar zu machen (Müller, 2014, 2015).

Auch die Organisation und die Strukturierung der Lernunterstützung lassen sich zu Merkmalen der Oberflächenstruktur zählen, beispielsweise die Präsenz von Speziallehrpersonen oder weiterem pädagogischem Fachpersonal wie etwa schulische Fachpersonen für Sonderpädagogik (in der Schweiz: Heilpädagoginnen und Heilpädagogen), DaZ-Lehrpersonen, Logopädinnen und Logopäden oder Schulsozialarbeiterinnen und Schulsozialarbeitern. Ebenfalls zu denken ist in diesem Zusammenhang an die organisationale Einbettung von individuellen Coaching-Gesprächen in den Stundenplan, in denen Lehrpersonen mit den Schülerinnen und Schülern ihre Lernprozesse reflektieren (u. a. Müller, 2014).

Weitere organisatorische Merkmale sind die zeitliche Strukturierung eines Unterrichtstages mithilfe von Stundenplänen, die Aufteilung der Klassen in alters- und/oder leistungsgemischte Lerngruppen und die Strukturierung der Lernräume beispielsweise in Lernlandschaften und Input-Räume (u. a. Kunter \& Trautwein, 2013; Reusser, 2008). Im Kontext der unterrichtszentrierten Schulentwicklung werden diese drei Merkmale jedoch nicht zur Entwicklungsdimension „Unterrichtsentwicklung in Richtung personalisierter Gestaltung von Lehr- und Lernprozessen“, sondern zur Dimension „Entwicklung organisationaler Strukturen“ (siehe Abschnitt 4.5.2) gezählt, weil deren Weiterentwicklung neben der Unterrichtsebene insbesondere die Schulebene tangiert. Das heißt, es werden nicht nur in einer einzelnen Klasse Phasen von geführtem und offenem Unterricht im Stundenplan definiert, Lerngruppen altersdurchmischt zusammengesetzt oder Lernlandschaften eingerichtet, sondern der Stundenplan, die Struktur der Lerngruppe sowie das Raumkonzept werden auf alle Klassen einer Schule angewendet (D. Hargreaves, 2006a; Prain et al., 2015a).

\section{Merkmale der Tiefenstruktur des Unterrichts}

Merkmale der Tiefenstruktur des Unterrichts beziehen sich auf didaktisches Handeln, das auf der psychologisch-didaktischen Qualitätsebene von Lernen basiert und kognitive, motivational-emotionale sowie soziale Lerndynamiken bei den Lernenden fördert (Reusser \& Pauli, 2010b). Empirisch erhärtet haben sich drei oft rezipierten Basisdimensionen guten Unterrichts „Effiziente Klassenführung“, 
„Unterstützendes Unterrichtsklima“ und „Kognitive Aktivierung“ (Klieme et al., 2006; Kunter \& Ewald, 2016; Kunter \& Trautwein, 2013; Praetorius et al., 2020):

- Unter der effizienten Klassenführung werden Handlungsweisen der Lehrperson subsumiert, mit denen eine effektive Lernzeitnutzung der Schülerinnen und Schüler gewährleistet wird, zum Beispiel Unterrichtsabläufe klar zu strukturieren, Verhaltensregeln einzuführen und einzufordern oder Störungen präventiv zu bearbeiten.

- Ein unterstützendes Unterrichtsklima umfasst die an den Schülerinnen und Schülern orientierte Lernunterstützung bei Problemen mit dem Verstehen des Lerngegenstands, bei der Lehrpersonen auf individuelle Lernpotenziale und Bedürfnisse eingehen. Wertschätzende und respektvolle Interaktionen mit positiver Beziehungsqualität und einer ko-konstruktiven Gesprächskultur prägen ein produktives Lernklima.

- Kognitive Aktivierung bedeutet, die Schülerinnen und Schüler zu kognitiven Aktivitäten anzuregen und anzuleiten, damit sie sich in Prozessen intensiver Informationsverarbeitung aktiv und elaboriert mit den Lerngegenständen auseinandersetzen, diese mit eigenen Wissens- und Denkstrukturen verbinden und gedankliche Umstrukturierungen vornehmen. In diesem Zusammenhang lässt sich der Begriff des Higherorder thinking nennen: Je stärker sich die Schülerinnen und Schüler aktiv mit dem Lerngegenstand kognitiv auseinandersetzen, desto besser werden dahinterstehende Wissens- und Denkstrukturen verstanden und desto nachhaltiger wird gelernt (u. a. Greeno, Collins \& Resnick, 1996). Die kognitive Aktivierung hängt u. a. mit der Aufgabenqualität und der in deren Bearbeitung stattfindenden Unterstützungsqualität zusammen.

Unterricht ist dann qualitätsvoll, wenn seine Gestaltung den drei Basisdimensionen und weiteren Tiefenqualitäten Rechnung trägt. Zu diesen gehören auch soziale Aspekte des Lernens, die insbesondere beim kooperativen Lernen zum Tragen kommen. Denn wenn Schülerinnen und Schüler in Partner- oder Gruppenarbeiten miteinander Lernaufgaben bearbeiten, ist die Interaktionsqualität in solch kooperativen Lerngemeinschaften bedeutsam, besonders wenn die Lehrperson die sozialen Lerndynamiken nicht immer einsehen und notfalls nicht gleich intervenieren kann. In diesem Zusammenhang stellt sich die Qualitätsfrage, inwiefern die Schülerinnen und Schüler am Thema arbeiten, auf Gesprächsimpulse eingehen, eigenes Wissen mit einbringen und dieses mit den Wissensbeständen der Mitschülerinnen und Mitschüler verknüpfen. In Unterrichtskonzepten, in denen Lehr- und Lernprozesse vermehrt personalisiert gestaltet werden, stellen sich solche Fragen insbesondere in den offenen Unterrichtsphasen, in denen die Schülerinnen und 
Schüler beispielsweise in der Lernlandschaft meist selbst entscheiden können, wann und mit wem sie welche Aufgaben lösen (u. a. Stebler et al., 2018).

In Auflistungen von Merkmalen guten Unterrichts lassen sich weitere Tiefenmerkmale finden (u. a. Helmke, 2017; Meyer, 2009; Reusser, 2008, 2009). Diese Arbeit lehnt sich an den Ausführungen von Reusser an, die auf einer ,psychologisch-didaktische[n] Grundvorstellung über den Zyklus vollständiger Lehr- [und] Lernprozesse“ gründen (Reusser, 2008, S. 231). Vollständige Lernzyklen können u. a. mit Aeblis (u. a. 1983/2006) PADUA-Modell beschrieben werden: „P“ und „A“ stehen für das Problemlösende Aufbauen, „D“ steht für das Durcharbeiten, „U“ für das Üben und Wiederholen und „A“ für das Anwenden einer kognitiven Struktur. Ziel des Unterrichts im Sinne der psychologischen Didaktik von Aebli ist der problemorientierte und verstehensbezogene Aufbau von beweglichem (intelligentem) Wissen. Das didaktische Handeln der Lehrperson auf der Inszenierungsebene des Unterrichts ordnet sich dieser psychologischen Lernebene unter (Messner \& Reusser, 2006; Reusser et al., 2013). Pädagogisch-psychologische Qualitätsmerkmale guten Unterrichts ordnet Reusser (2011) entlang der drei Teilkulturen seines didaktischen Dreiecks (siehe Abschnitt 4.5.1.2) an (Abbildung 4.7):

Hervorzuheben ist diesbezüglich, dass erstens mit den Kriterien der Ziel- und Stoffkultur die fachdidaktischen Merkmale mitbeachtet werden, deren Fehlen in den meisten allgemeindidaktischen Arbeiten in jüngeren Publikationen kritisiert wird (Lipowsky \& Bleck, 2019; Messner, 2019). Sinnstiftendes und verstehensbezogenes Lernen gründet auf der Qualität der fachlich relevanten Lerninhalte, der Lernziele sowie der Lernaufgaben. Zudem sind in den psychologischen Dimensionen (Lehr- und Lernkultur) neben den bereits genannten Merkmalen die Förderung von Lernstrategien und das Lernen-Lernen zu erwähnen. In personalisierten Lernumgebungen, die darauf ausgerichtet sind, dass Schülerinnen und Schüler vermehrt selbstständig und eigenverantwortlich lernen, müssen diese für die eigene Gestaltung der Lernprozesse befähigt werden. Sie benötigen daher Strategien, um ihre Lernzeit zu organisieren, sich zu motivieren und auch in anstrengenden Phasen den Durchhaltewillen aufzubringen, an den Aufgaben weiterzuarbeiten.

Bei alledem ist von zentraler Bedeutung, dass solcherart qualitätsvolles Lernen auf eine starke Lernunterstützung durch die Lehrpersonen angewiesen ist. Hervorzuheben sind somit ebenfalls die personalen und sozialen Dimensionen (Interaktions- und Unterstützungskultur) einer individuellen Lernunterstützung in personalisiert gestalteten Lernkontexten. Deren Ziel ist es, systematisch Strukturen einzurichten, um die Schülerinnen und Schüler je nach ihren Bedürfnissen und Wünschen in ihrem Lernen zu unterstützen und in Coaching-Gesprächen 
Schwerpunkt Unterrichtsentwicklung Forschungsbasierte Qualitätsmerkmale

\section{Ziel- und Stoffkultur}

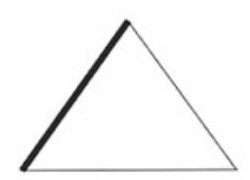

Lehr-Lernkultur

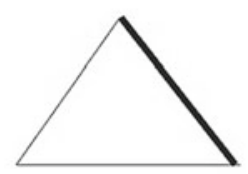

Interaktions- und Unterstützungskultur

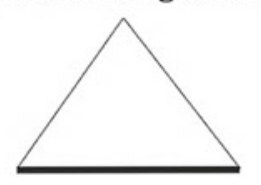

1. Fachstandards und Stoffqualität

2. Klarheit über fachliche und überfachliche Bildungsziele, Kompetenzen und Erwartungen

3. Qualität von Lehrmitteln, Lernaufgaben und Lernmedien

4. Effiziente Klassenführung und Lernzeitstrukturierung

5. Angemessene Methoden- und Inszenierungsvielfalt

6. Qualität des Strukturaufbaus im Sinne von Verstehensklarheit, intelligentem Üben und Lerntransfer

7. Motivierende und kognitiv aktivierende Lernimpulse, Fachaufgaben und Lernsituationen

8. Sinnstiftende Unterrichtsgespräche, dialogische und diskursive Unterrichtsführung

9. Förderung von Lernstrategien, Lernen lernen

10. Lernförderliches Sozial- und Interaktionsklima

11. Durch persönliche Zuwendung geprägte LehrerSchüler-Beziehung

12. Lerndiagnostisch fundierte individuelle Lernunterstützung und adaptives Coaching

Abbildung 4.7 Forschungsbasierte Qualitätsmerkmale guten Unterrichts (Reusser, 2011)

ihre Lernprozesse zu reflektieren (u. a. Müller, 2014). Als Modell für eine lernwirksame Lernunterstützung wird in der pädagogisch-psychologischen Lehr- und Lernforschung oftmals die kognitive Meisterlehre respektive cognitive apprenticeship angeführt (Collins et al., 1989). Das an Vygotskij (u. a. 1934/2002) angelehnte fünfstufige Modell beschreibt eine Synthese von professionellem Lehrpersonenhandeln und dem Ausmaß an Selbstständigkeit der Schülerinnen und Schüler in ihrem fachlichen Lernen: (1) Die Lehrperson veranschaulicht durch lautes Denken und Beobachtbarmachen die kognitiven Zieltätigkeiten (z. B. Lösen eines Problems) (modeling). (2) Anschließend leitet die Lehrperson in den Problemlöseversuchen der Lernenden an. Sie nimmt die Rolle einer Trainerin ein, steuert und überwacht die Lerntätigkeiten und unterstützt situationsbezogen (coaching). (3) Als „Lerngerüst“" gibt die Lehrperson Hilfestellungen, die in Interaktion zwischen Lehrperson und Lernenden schrittweise ,zurückgebaut“ werden. Die Schülerinnen und Schüler lösen sich allmählich von der angeleiteten Lerntätigkeit, übernehmen immer mehr Verantwortung und treffen zunehmend eigene Entscheidungen (scaffolding). (4) Die Lehrperson zieht sich mit der Zeit 
zurück und gibt die Lernsteuerung graduell frei (fading). (5) Die Schülerinnen und Schüler werden angehalten, ihre Lernprozesse und Lernprodukte zu reflektieren (reflection).

\subsubsection{Empirische Befunde zu Entwicklungstätigkeiten in Schulen mit personalisierten Lernkonzepten}

In den vorherigen Unterkapiteln (siehe Abschnitt 4.5.1.2 und Abschnitt 4.5.1.3) wurde dargestellt, welche lernorganisatorischen Strukturen und welches didaktische Handeln vor dem Hintergrund eines kognitiv- und soziokonstruktivistischen Lernverständnisses (siehe Abschnitt 4.5.1.1) für verstehensbezogene Lernprozesse relevant sind, in denen sich Schülerinnen und Schüler mit Lerninhalten aktiv auseinandersetzen und diese tiefgehend durchdringen. Nachfolgend steht die Frage im Zentrum, welche dieser Unterrichtsmerkmale bearbeitet werden, wenn Schulleitende und Lehrpersonen Konzepte personalisierten Lernens einführen und weiterentwickeln. Hierfür wird der Forschungsstand aus Studien rezipiert, in denen mehrheitlich Unterrichtskonzepte mit vermehrt personalisiert gestalteten Lehr- und Lernprozessen untersucht werden. Ergänzt werden die Ergebnisse mit Erkenntnissen aus Publikationen, in denen die konzeptionelle Umsetzung von personalisierten Lernkonzepten teils theoretisch fundiert, aber auch in Form von erfahrungsbasierten Fallberichten beschrieben wird.

Teil des perLen-Forschungsprojekts (siehe Abschnitt 1.2) war eine längsschnittliche Fragebogenerhebung in 65 Schulen der Deutschschweiz. Ein Forschungsziel bestand darin, Varianzen in der didaktischen Gestaltung aufzuzeigen. Hierfür wurden, aufbauend auf den von Lehrpersonen gegebenen Einschätzungen des Personalisierungsgrads, drei Gruppen von Schulen gebildet. ${ }^{3}$ In der

3 „Um das Ausmaß der Orientierung an personalisiertem Lernen der Schule valid zu eruieren, wurden die Lehrpersonen zweimal (Schuljahr 2013/14 und Schuljahr 2014/15) jeweils via dieselbe Frage bewusst um eine sehr globale, subjektive Einschätzung gebeten: Wie stark orientiert sich ihre Schule an personalisierten Lernkonzepten? (Antwortformat: $++(4),+$ (3), - (2), - - (1)). Mit den Antworten wurden zuerst pro Schule und Befragungszeitpunkt ein Mittelwert und anschließend die Korrelation der Mittelwerte aus der ersten $\left(M_{l}\right.$; Schuljahr 2013/14) und der zweiten Befragung $\left(M_{2}\right.$; Schuljahr 2014/15) berechnet $(r=.67)$. Danach wurde aus den beiden Mittelwerten für jede Schule nochmals der Mittelwert gebildet $\left(M_{I}\right.$ $+M_{2} / 2$ ). Anhand dieses Mittelwerts (,Personalisierungsgrad der Schule') wurden die 65 perLen-Schulen in drei ähnlich große Gruppen aufgeteilt: Gruppe 1 (G1): $M \geq 3.3$; diese Schulen bezeichnen wir im Folgenden als, sehr stark personalisierte Schulen ', Gruppe 2 (G2): $3 \leq M<3.3$; wird als , stark personalisierte Schulen ' bezeichnet und Gruppe 3 (G3): $M<3$; als ,moderat personalisierte Schulen'. Die Einteilung wurde durch die perLen-Forschungsgruppe anhand von Schuldokumenten (u. a. Schulprogramm) und Feldkenntnis (u. a. Schulbesuche, Interviews mit Schulleitungen und Lehrpersonen) überprüft“ (Stebler et al., 2018, S. 168). 
Datenauswertung zeigt sich, dass in sehr stark personalisierten Schulen häufiger offene Unterrichtsphasen mit selbstständigem und eigenverantwortlichem Lernen stattfinden als in moderat personalisierten Schulen. Des Weiteren führten die Lehrpersonen in sehr stark personalisierten Schulen häufiger individuelle Lernunterstützung, -beratung und fachliches Coaching durch. Die Schülerinnen und Schüler in diesen Schulen arbeiten häufiger mit individuellen Lernzielen und mit Lernjournalen, in denen sie ihre Lernprozesse planen und/oder dokumentieren (Stebler et al., 2017, 2018).

In den 23 Schulen der US-amerikanischen Studie der RAND Corporation (2014) nutzten Lehrpersonen Instrumente, mit denen sie individuelle Bedürfnisse, Stärken, Motivation und Ziele der Schülerinnen und Schüler diagnostizierten und dokumentierten. Auch die Schülerinnen und Schüler selbst nutzten solche Instrumente für die Planung und die Reflexion ihrer personalen Lernwege. Des Weiteren wurden sie häufiger individuell unterstützt und beraten und konnten zwischen Formen der Einzel-, Partner- und Gruppenarbeit wählen.

Die Studie von Judy Sebba et al. (2007) zeigt auf, dass spezifische Curricula und Stoffpläne erarbeitet werden, in denen die Lehrpersonen die Auswahl und die Abfolge der Lerninhalte an die heterogenen Lerngruppen anpassen. Zudem können die Schülerinnen und Schüler ihre gelösten Aufgaben selbst kontrollieren oder diese mit anderen Schülerinnen und Schülern gemeinsam überprüfen (peer assessments). Sie haben vermehrt Wahlmöglichkeiten in Bezug darauf, wo (Platz für Einzelarbeit oder Gruppenarbeit) und mit wem sie Lernaufgaben lösen möchten.

Zusammengefasst lässt sich sagen, dass die Lernumgebungen in den untersuchten Schulen teils stark von einem eng geführten Klassenunterricht abweichen. So gibt es sowohl variabel geführte Unterrichtsphasen als auch offene Sequenzen. In den offenen Phasen können die Schülerinnen und Schüler zwischen verschiedenen Lernformen, Lernorten und Lerninhalten wählen und haben die Möglichkeit, im gewissen Ausmaß Lernaufgaben in ihrem eigenen Tempo zu lösen. Zudem zeichnen sich die Schulen durch vermehrt individuelle Lernunterstützung, Lernberatung und individuelles Lerncoaching aus. Im Kollegium werden gemeinsam Stoffpläne erstellt, die es ermöglichen, in einer heterogenen Lerngruppe Lerninhalte bereitzustellen, die den individuellen Voraussetzungen und Anforderungen der Schülerinnen und Schüler entsprechen. Dies ermöglicht es den Lehrpersonen, auch trotz verschiedener Alters- und/oder Niveaustufen die Übersicht zu bewahren und individuelle Lernziele zu definieren.

Ein weiterer Punkt sind die Dokumentation und die Verwaltung der personalisierten Lernprozesse: Die Schülerinnen und Schüler führen beispielsweise Lernjournale auf Papier, um ihr Lernen zu planen und zu dokumentieren. Teilweise 
werden ICT-Lösungen eingesetzt, mit denen Lehrpersonen und teilweise auch Schülerinnen und Schüler Lernziele festlegen, Lernaufgaben aufteilen und die Arbeitsfortschritte dokumentieren. So erhalten die Lehrpersonen eine Übersicht über die verschiedenen Arbeitsstände und über die erbrachten Leistungen.

Diese Merkmale lassen sich auch in den konzeptionellen Arbeiten, die teils auf theoretischen Überlegungen, teils auf Erfahrungsberichten beruhen, von D. Hargreaves (2006a), Bray und McClaskey (2015) oder des Department for Children Schools and Families (2008) wiederfinden. Zusätzlich wird in den drei Publikationen die Leistungsbeurteilung thematisiert: Lernergebnisse in einer heterogenen Lerngruppe mit standardisierten, auf eine Gruppennorm bezogenen Instrumente einzuschätzen, ist sehr herausfordernd. Es braucht daher Instrumente der summativen und formativen Leistungsbeurteilung, mit denen Lernfortschritte überprüft und evaluiert werden können, damit sich darauf aufbauend nächste Lernschritte planen lassen. Besonders die formative Leistungsbeurteilung wird positiv bewertet, weil sie vermehrt auf das Lernen individueller Schülerinnen und Schüler und dessen Förderung ausgerichtet ist. D. Hargreaves (2006a) sowie Bray und McClaskey (2015) zählen zusätzlich das Erlernen von Lernstrategien auf, mit denen die Schülerinnen und Schüler befähigt werden, in offenen Lernsettings selbstständig und eigenverantwortlich zu lernen.

Forschungsergebnisse aus dem deutschen Sprachraum zu erweiterten Lehrund Lernformen (u. a. Reusser et al., 2010) oder zum offenen Unterricht (u. a. Forschungsüberblick von Bohl \& Kucharz, 2010) zeigen im Vergleich mit den englischsprachigen Publikationen sehr ähnliche Ergebnisse. Lehrpersonen organisieren Lerngelegenheiten mit vielfältigen Lehr- und Lernformen, in denen vermehrt Autonomiespielräume für selbstständiges Planen, Durchführen, Evaluieren und Reflektieren individueller Lernaktivitäten vorhanden sind. Allerdings werden in den oben aufgeführten englischsprachigen Arbeiten die pädagogisch-psychologischen Prozessqualitäten von Lernen weniger thematisiert als in Publikationen aus dem deutschsprachigen Raum. In Videostudien der Forschungsgruppe um Kurt Reusser und Christine Pauli (Pauli et al., 2008; Pauli $\&$ Reusser, 2006; Pauli et al., 2003; Reusser et al., 2010) wurde auch die Tiefenstruktur von erweiterten Lehr- und Lernformen fokussiert. Guter Unterricht zeichnet sich diesen Analysen dadurch aus, dass Lehrpersonen Gelegenheiten schaffen, bei denen sich die Schülerinnen und Schüler aktiv und eigenständig mit fachlichen Problemstellungen auseinandersetzen können und von Lehrpersonen kognitiv-aktivierend unterstützt werden. Die Ergebnisse einer internationalen und schweizerischen Videostudie zum Mathematikunterricht zeigen, dass sowohl die Schülerinnen und Schüler als auch unabhängige Expertinnen und Experten den offenen Unterricht hinsichtlich der Tiefenmerkmale kognitiver Aktivierung und 
Orientierung an den Schülerinnen und Schülern tendenziell besser einschätzen als eng geführten Klassenunterricht. Allerdings ließen sich keine Leistungsverbesserungen nachweisen. Dies wurde u. a. damit erklärt, dass die Aufgabenqualität (z. B. Komplexität, Alltagsbezug, Bearbeitungsdauer etc.) für offene Unterrichtsphasen verbesserungswürdig sei (Reusser \& Pauli, 2010a). Für eine Schul- und Unterrichtsentwicklung in Richtung personalisierten Lernens sind Lernaufgaben bedeutsam (z. B. Problemlöseaufgaben), die selbstständig bearbeitbar sind und von denen alle Schülerinnen und Schüler motiviert und kognitiv herausgefordert werden (Reusser, 2017).

Tiefenmerkmale von Unterricht werden auch in der für die vorliegende Arbeit wichtigen Teilstudie des Begleitforschungsprojekts WissGem in BadenWürttemberg untersucht (Meissner et al., 2016). Die Autorinnen und Autoren verfolgten ähnliche Fragestellungen (Unterscheidung Oberflächen- und Tiefenstruktur) und arbeiteten mit den gleichen Instrumenten der Datenerhebung (teilstrukturierte Leitfadeninterviews) und Datenauswertung (strukturierende Inhaltsanalyse). Im Projekt WissGem wurden in einem umfangreichen Schulentwicklungsprojekt Gemeinschaftsschulen implementiert, in denen teils Konzepte personalisierten Lernens eingeführt wurden (Zylka, 2017). In der Teilstudie wurden 53 teilstrukturierte und leitfadengestützte Interviews mittels strukturierender Inhaltsanalyse hinsichtlich der Gestaltung und der Entwicklung des Unterrichts ausgewertet. Die Antworten wurden im Hinblick auf Merkmale der Oberflächen- und Tiefenstruktur des Unterrichts codiert. Interviewaussagen zur Oberflächenstruktur bezogen sich beispielsweise auf die eingesetzten Lehr- und Lernformen, u. a. auf den Grad und den Umfang der Lehrpersonenzentrierung, des individualisierten Unterrichts und der inneren Differenzierung. Aussagen zur Tiefenstruktur thematisierten die Klassenführung, das Nutzungsverhalten der Schülerinnen und Schüler oder das Lern- und Unterrichtsklima. 68.6 Prozent der Aussagen ließen sich Aspekten der Tiefenstruktur des Unterrichts zuordnen und 31.4 Prozent der Aussagen Aspekten der Oberflächenstruktur (Meissner et al., 2016).

\subsubsection{Entwicklung organisationaler Strukturen}

Für die Entfaltung tiefenstruktureller Lernqualitäten sind unterstützende organisationale Strukturen erforderlich (Reusser, 2019): Damit Schülerinnen und Schüler auf eigenen Wegen lernen können, braucht es entsprechend organisierte Lernumgebungen mit ausreichender Lernzeit sowie Lernräume mit Zonen für Einzelund Gruppenarbeiten. Traditionelle Schul- und Unterrichtsstrukturen, in denen eine Lehrperson eine (vermeintlich) homogene Lerngruppe im 45-Minuten-Takt 
unterrichtet, sind dafür kaum geeignet (Bray \& McClaskey, 2015; Müller, 2015). Diese seit Jahrzehnten unveränderte Schul- und Unterrichtsorganisation, auch als grammar of schooling (Tyack \& Tobin, 1994) bezeichnet, prägt die Vorstellungen und das Verständnis von Lehrpersonen, wie Schule und Unterricht „funktionieren“, auch heute noch in vielen Fällen. Daher geht die Weiterentwicklung solch fundamentaler Strukturen meist mit einer Veränderung des Schul- und Unterrichtsverständnisses einher, was wiederum einen großen Ressourcen- und Zeitaufwand erfordert (Tyack \& Tobin, 1994).

In diesem Kapitel steht vor diesem Hintergrund die Frage im Mittelpunkt, wie die organisationalen Strukturen in Schulen mit Konzepten personalisierten Lernens gestaltet sind. In der Literatur lassen sich diesbezüglich vier Strukturelemente identifizieren, die nachfolgend beschrieben werden (u. a. Bray \& McClaskey, 2015; Prain et al., 2015a; RAND Corporation, 2014):

- Lernräume (räumliche Strukturierung),

- Stundenpläne (zeitliche Strukturierung),

- Zusammensetzung der Lerngruppen sowie

- Team- und Kooperationsstrukturen der Lehrpersonen.

\section{Lernräume (räumliche Strukturierung)}

Unterrichtsräume, in denen Lehrpersonen und Schülerinnen und Schüler zwischen verschiedenen Lehr- und Lernformen situativ und meist individuell wechseln können, müssen anderen Anforderungen genügen als Räume für klassischen Frontalunterricht. In der Alemannenschule Wutöschingen beispielsweise, einer Gemeinschaftsschule in Baden-Württemberg (Deutschland), wurde ein neues Schulgebäude unter Berücksichtigung des Unterrichtskonzepts gebaut. Bei der Gestaltung und der Einrichtung der Räume wurde auf deren jeweilige Funktion geachtet. So soll im Lernatelier leise und selbstständig gearbeitet werden (Abbildung 4.8). Aus diesem Grund wurden helle und sanfte Farben für eine ruhige Lernatmosphäre gewählt. Dagegen sind im sogenannten „Marktplatz“, der für kooperatives Lernen genutzt wird, kräftigere Farben und eine größere Farbpalette vorzufinden (Abbildung 4.9). Die Möbel können flexibel verschoben und der Gruppengröße angepasst werden. Ein dritter Bereich sind die Inputräume, in denen geführte Unterrichtsphasen stattfinden (Abbildung 4.10) (Schöler \& Schabiger, 2017).

Ähnliche Raumstrukturen lassen sich auch in einigen Schulen in der Schweiz finden. In einer Fallstudie des perLen-Projekts untersucht Bachmann (2015a) Lernräume von drei Schulen mit Konzepten personalisierten Lernens. Aus ihren 


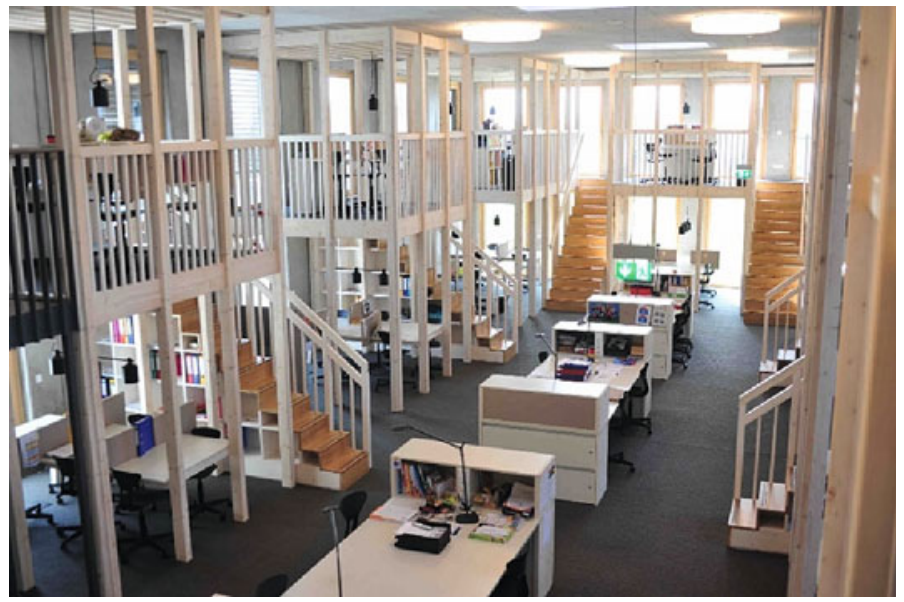

Abbildung 4.8 Lernatelier (Bildquelle: Gemeinde Wutöschingen/Alemannenschule Wutöschingen)

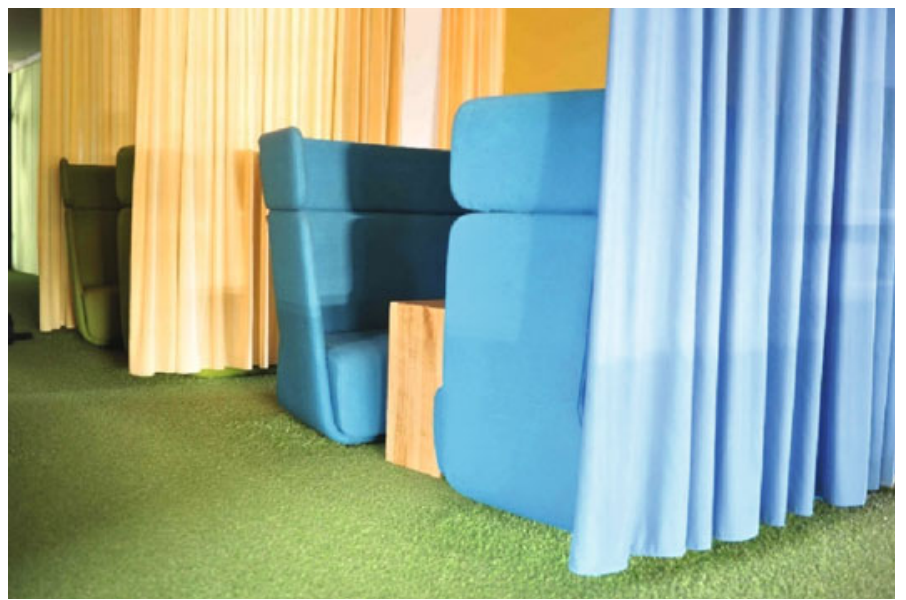

Abbildung 4.9 Marktplatz (Bildquelle: Gemeinde Wutöschingen/Alemannenschule Wutöschingen) 


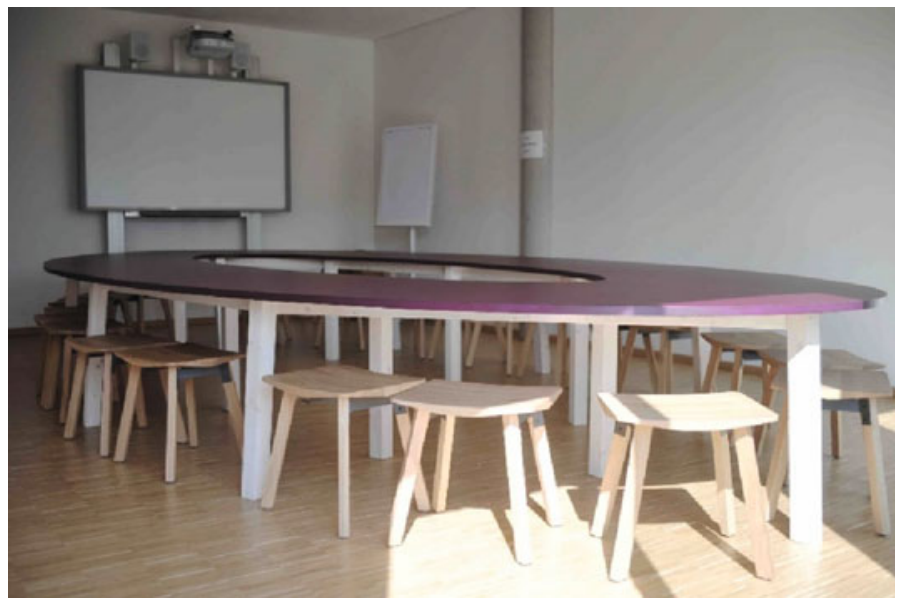

Abbildung 4.10 Inputraum (Bildquelle: Gemeinde Wutöschingen/Alemannenschule Wutöschingen)

Befunden wird deutlich, dass ein vielfältiges Raumangebot für Einzel-, Partneroder Gruppenarbeit, Plenumsarbeit oder Coaching-Gespräche geschaffen wurde. Die Raumangebote und die Raumgestaltung sind in den Schulen unterschiedlich und hängen stark von der verfügbaren Raumkapazität in jeder Schule ab. Je mehr Räume genutzt werden können, desto mehr Differenzierungen gibt es zwischen den Räumen. In einigen Schulen werden sogenannte „Lernlandschaften“ eingerichtet, in denen jede Schülerin bzw. jeder Schüler über einen eigenen Arbeitsplatz verfügt (Abbildung 4.11). Oft werden auch Flure für kooperative Lernphasen genutzt (Abbildung 4.12). Sind nicht genügend einzelne Räume vorhanden, wird ein Lernraum in verschiedene Lernzonen aufgeteilt (Abbildung 4.13) (Bachmann, 2015a).

Im Vergleich zur Alemannenschule Wutöschingen, bei der das Schulhaus unter Berücksichtigung des Unterrichtskonzepts erbaut wurde, ist deutlich zu erkennen, dass in den perLen-Schulen Lehrpersonen und Schulleitende die vorhandenen Raumkapazitäten kreativ nutzen und bearbeiten müssen, um personalisiertes Lernen zu ermöglichen. Auch in weiteren Schulen des perLen-Projekts sowie in Schulen mit Konzepten personalisierten Lernens aus dem angloamerikanischen Bildungsraum werden Lernräume reorganisiert und flexibler genutzt. Unter anderem lassen sich Schulen finden, in denen Räume doppelt genutzt werden, zum einen für Phasen des selbstständigen Lernens an individuellen Lerngegenständen 


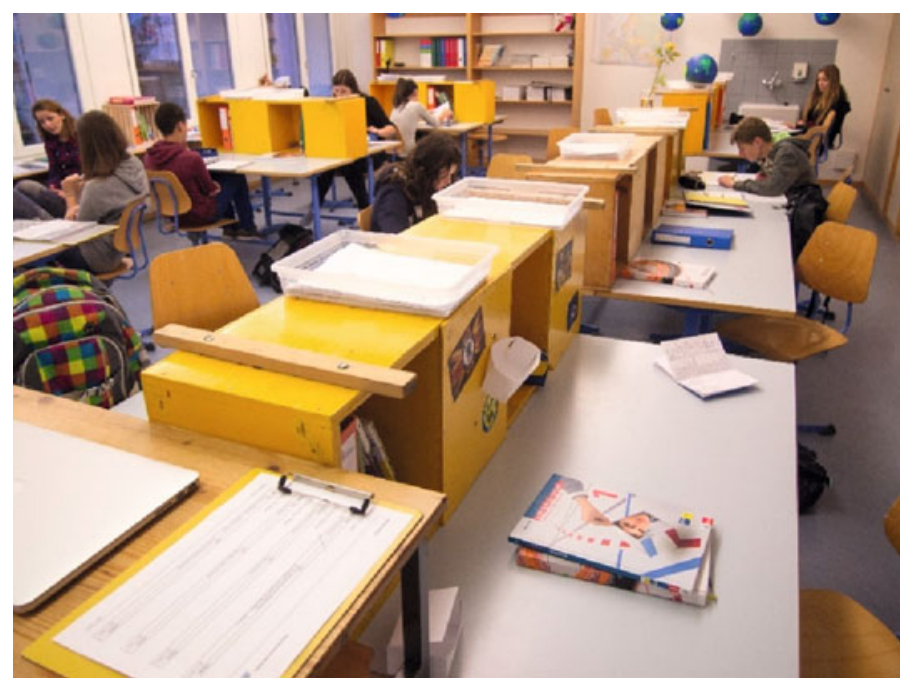

Abbildung 4.11 Lernlandschaft mit persönlichen Arbeitsplätzen (Bildquelle: Bachmann, 2015a)

und zum anderen für geführten Frontalunterricht. Dies kann nacheinander oder zur selben Zeit stattfinden. Hierfür sind Möblierungen notwendig, die durch Umstellen oder/und Stapeln die jeweiligen Lehr- und Lernformen ermöglichen (Pauli et al., 2018; Pauli et al., 2017; Prain et al., 2015a, 2015b; RAND Corporation, 2014; Stebler et al., 2017, 2018).

Neben der Möblierung sind digitale Medien, sowohl die Hard als auch die Software, ein weiteres Strukturmerkmal. In fast jedem Klassenzimmer sind Computer vorhanden. Darüber hinaus verfügen einige Schulen über interaktive Whiteboards oder Tablets. Der Zugang zum Internet sowie Lernprogramme, die Lernaufgaben für Schülerinnen und Schüler automatisieren und anhand verschiedener Daten (Leistungsniveau, Bearbeitungszeit bisheriger Aufgaben, Anzahl richtig gelöster Aufgaben etc.) zusammenstellen, unterstützen das individuelle Lernen. Zusätzlich werden in einigen Schulen Programme eingesetzt, mit denen die individuellen Lernprozesse organisiert und dokumentiert werden können (u. a. Petko et al., 2017; RAND Corporation, 2014). 


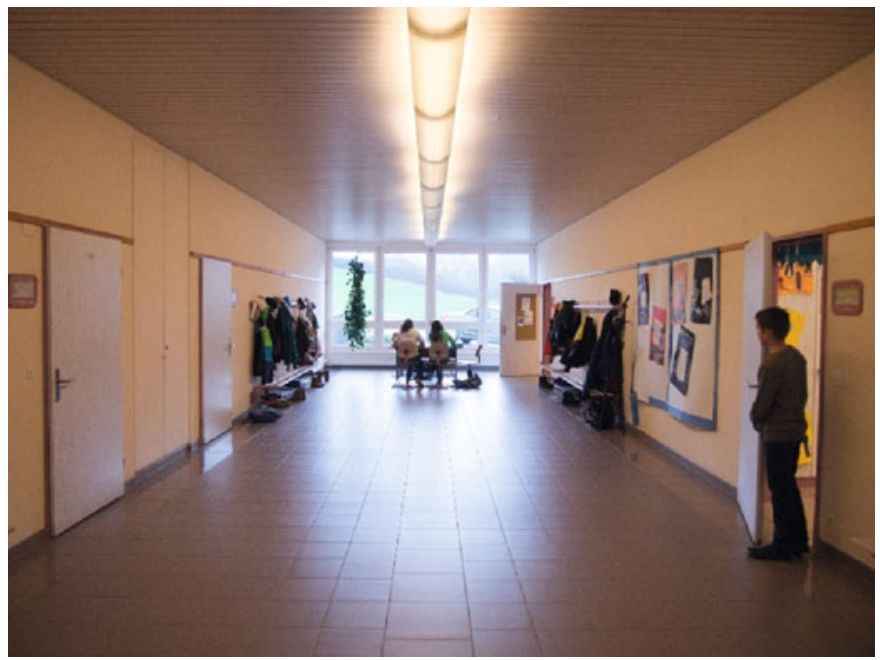

Abbildung 4.12 Aufgrund begrenzten Platzes werden die weiträumigen Flure als Lernort benutzt (Bildquelle: Bachmann, 2015a)

\section{Stundenpläne (zeitliche Strukturierung)}

Auch zeitliche Strukturen können sich mit der Einführung von Konzepten personalisierten Lernens ändern. Achermann und Gehrig (2013) haben sieben Primarschulen (1.-6. Klasse) in der Schweiz mit altersdurchmischten und individualisierten Lernkonzepten diesbezüglich verglichen. Einen Fächerstundenplan gab es in den Schulen nicht mehr. Stattdessen hatte sich eine Wochenstruktur etabliert, in der es nur noch selten eine 45-Minuten-Lektion gab (Abbildung 4.14). In der Begleitforschung von Prain et al. (2015c) dauerten die Unterrichtssequenzen der untersuchten Schulen hingegen zwischen 35 und 120 Minuten, wobei die Lehrpersonen 70 Minuten als am sinnvollsten ansahen.

Die in Abbildung 4.14 aufgeführten „Kurse“ sind geführter Unterricht, der von einer oder mehreren Lehrpersonen erteilt wird. Unter „Plan“ ist das selbstständige und eigenverantwortliche Lernen im offenen Unterricht $\mathrm{zu}$ verstehen, ,in [dem] die Kinder nach ihren Bedürfnissen und Interessen lernen und tätig sind“ (Achermann \& Gehrig, 2013, S. 70). Auch die in den Forschungsprojekten WissGem (Forschungsgruppe WissGem, 2016) und perLen (Stebler et al., 2018) untersuchten Schulen unterteilen ihren Stundenplan in offenen und geführten Unterricht. Bei letzterer Studie zeigte sich, dass diese beiden Unterrichtsformen in einem 


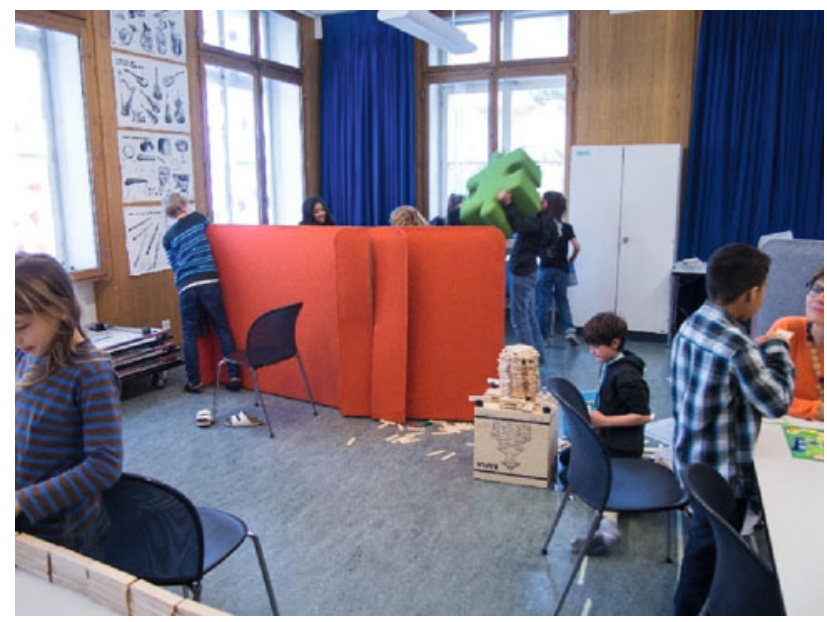

Abbildung 4.13 Raum für offenen Unterricht mit verschiedenen Lernzonen (Bildquelle: Bachmann, 2015a)

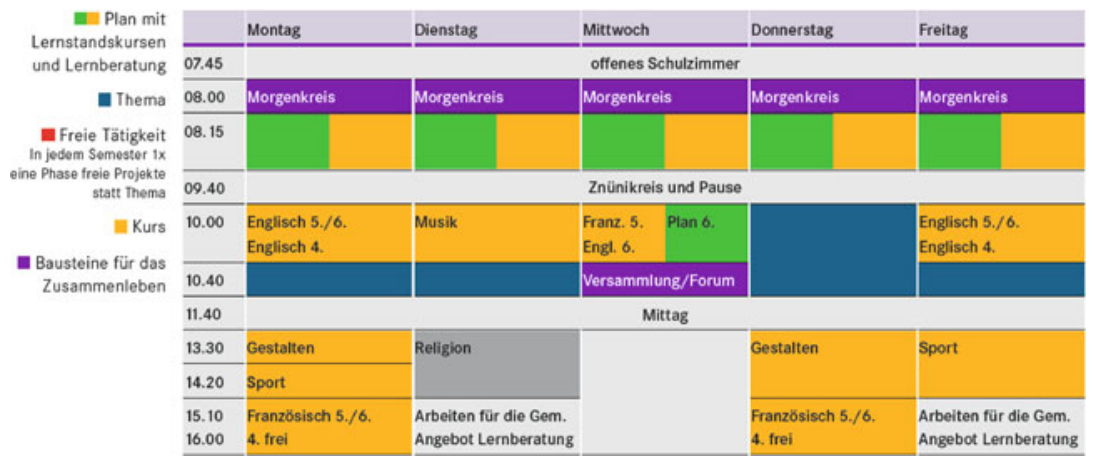

Abbildung 4.14 Wochenstruktur für drei parallele AdL-Klassen der Mittelstufe (4.-6. Klasse; AdL = Altersdurchmischtes Lernen) (nach: Achermann \& Gehrig, 2013)

Verhältnis von ca. 1:2 zueinanderstehen. Somit kam der geführte Unterricht nach wie vor häufiger vor als der offene Unterricht (Pauli et al., 2018; Pauli et al., 2017; Stebler et al., 2017, 2018). 


\section{Zusammensetzung der Lerngruppen}

Die Lerngruppen in den offenen Unterrichtssequenzen sind häufig heterogen. Sie setzen sich aus Schülerinnen und Schülern unterschiedlichen Alters und/oder verschiedener Leistungsniveaus zusammen. Zudem können die Lerngruppen grösser sein als im Klassenunterricht: In Lernlandschaften sind zum Beispiel Strukturen für mehr als 30 Schülerinnen und Schülern vorzufinden. In geführten Sequenzen sind die Lerngruppen im Gegensatz dazu eher leistungs- und/oder altershomogen (Achermann \& Gehrig, 2013; Müller, 2014; Pauli et al., 2018; Pauli et al., 2017; RAND Corporation, 2014; Reusser et al., 2015b; Stebler et al., 2017, 2018).

\section{Team- und Kooperationsstrukturen}

Die Neustrukturierung der Lerngruppen fördert und bedingt auch eine Neustrukturierung der Lehrpersonenkooperation. Wenn beispielsweise Jahrgangsklassen für offene Unterrichtsphasen in Lernlandschaften zusammengelegt werden, sind nicht nur die Lerngruppen grösser, sondern auch mehr Lehrpersonen für den Unterricht verantwortlich. Sie brauchen Zeit und Raum, um ihren alltägliche Unterrichtsarbeit zu organisieren und sich abzusprechen. Die Konzepte personalisierten Lernens werden zum Teil in denselben Teams, daneben aber auch in anderer Zusammensetzung ausgearbeitet und weiterentwickelt.

Gut vernetzte und enge Kooperationsstrukturen, in denen vermehrt unterrichtsbezogen und ko-konstruktiv zusammengearbeitet wird, sind in traditionell organisierten Schulen kaum vorzufinden. Solche Schulen lassen sich eher als lose gekoppeltes System (Weick, 1982) charakterisieren. Dies fördere ein Arbeitsklima, in dem Lehrpersonen sich eher allein für ihre Klasse und ihren Unterricht verantwortlich fühlten und weniger im Team zusammenarbeiten würden. Dieses Selbstverständnis wird auch als „Autonomie-Paritäts-Muster“ (Lortie, 1975) bezeichnet (siehe Abschnitt 4.3). Für die Entwicklung und die Umsetzung von Konzepten personalisierten Lernens, in der intensive Kooperation eine zentrale Voraussetzung ist (siehe Abschnitt 4.5.3), ist das Verständnis einer Lehrperson als „Einzelkämpferin“ oder „Einzelkämpfer“ jedoch kaum förderlich. Damit sich Lehrpersonen vermehrt als „Teamplayer" verstehen und eng zusammenarbeiten können, bedarf es transparenter kooperationsfördernder Strukturen (u. a. Idel \& Ullrich, 2013; Keller-Schneider \& Albisser, 2013; Köker, 2012; Lortie, 1975). Solche Strukturen können beispielsweise Fach-, Stufen- oder Jahrgangsteams umfassen, die Entwicklungsaufträge erhalten oder sich diese selbst geben und in Sitzungen Entwicklungsprojekte vorantreiben. Solche Teams dokumentieren ihre Arbeitsprozesse, haben klar definierte Arbeitsbereiche und Zuständigkeiten und vernetzen sich eng mit anderen Teams. Ergebnisse der Gruppen werden im Schulgremium, zum Beispiel in der Schulkonferenz und der Steuergruppe, vorgestellt, 
diskutiert und angepasst. Darüber hinaus werden in den Gremien Entwicklungsvorhaben definiert und an temporäre Untergruppen delegiert, die zu dem Thema in einem definierten Zeitrahmen ein Konzept oder Projekt ausarbeiten (Forschungsgruppe WissGem, 2016). Flankierende und unterstützende Elemente sind neben verbindlichen Arbeitszeiten im Team und der Verfügbarkeit von Arbeitsräumen auch begleitete Fortbildungs-, Coaching- oder Beratungsangebote sowie Unterstützung durch das Kollegium und die Schulleitenden (Arndt, 2016; Baumann, Henrich \& Studer, 2013; Kreis et al., 2016; Maag Merki et al., 2010; Reusser et al., 2013).

\subsubsection{Entwicklung der Kooperation zwischen Lehrpersonen}

Kooperation zwischen Lehrpersonen ist eine notwendige Bedingung wirksamer Unterrichts- und Schulentwicklung (Bonsen \& Frey, 2014; Haenisch \& Steffens, 2017; Holtappels, 2016; Saalfrank, 2016). Durch den kooperativen Einbezug verschiedener Wissens- und Erfahrungsbestände können komplexe Aufgaben und damit einhergehende Herausforderungen umfassender bearbeitet werden (Buddeberg, Wendt, Hornberg \& Bos, 2014; Trumpa, Franz \& Greiten, 2016). Wie im vorhergehenden Abschnitt festgehalten wurde, ist Kooperation auch in einer unterrichtszentrierten Schulentwicklung in Richtung personalisierten Lernens zentral. Doch wie lässt sich eine solche Kooperation charakterisieren? Um diese Frage zu klären, werden nachfolgend zuerst verschiedene Ansätze der Lehrpersonenkooperation skizziert. Anschließend werden die Kooperationsformen von Gräsel, Fussangel und Pröbstel (2006b) und deren Weiterentwicklungen beschrieben und es wird das Potenzial einer ko-konstruktiven und unterrichtsbezogenen Kooperation aufgezeigt. Am Schluss des Kapitels wird auf die Frage eingegangen, wie Lehrpersonen in Schulen mit Konzepten personalisierten Lernens zusammenarbeiten.

\section{Kooperationstypologien}

Je nach Definition und Merkmalen von Kooperation können verschiedene Formen unterschieden werden (Ahlgrimm, Krey \& Huber, 2012). So unterscheidet Little (1990) zwischen folgenden Formen:

- Gespräche über den Unterricht führen (story telling),

- Ratschläge und Hilfestellungen erteilen (aid and assistance),

- Austausch von Materialien und Methoden (sharing) und

- gemeinsame Entwicklung von Unterricht (joint work). 
Im deutschen Bildungsraum differenziert etwa Rolff (1993) zwischen gefügeartiger und teamartiger Kooperation, Esslinger (2002) zwischen struktureller und integrativer Kooperation, während Helmke und Jäger (2002) zwischen fachübergreifendem und fächerverbindendem Lernen, gemeinsamer Unterrichtsvorbereitung sowie gemeinsamer Unterrichtsdurchführung und Steinert et al. (2006) zwischen Fragmentierung, Differenzierung, Koordination, Interaktion und Integration unterscheiden.

\section{Austausch, Arbeitsteilung und Ko-Konstruktion}

Im deutschsprachigen Raum werden die Kooperationsformen von Gräsel et al. (2006a) am häufigsten rezipiert und weiterentwickelt. Sie unterscheiden zwischen (1) Austausch von beruflichen Materialien und Ideen zur Informationsweitergabe ohne gemeinsame Zielsetzung, (2) arbeitsteiliger Kooperation, bei der Aufgaben unter einer gemeinsamen Zielsetzung aufgeteilt werden, um Arbeitsprozesse effizienter zu gestalten, und (3) Ko-Konstruktion, die in einem intensiven, auf ein gemeinsames Ziel gerichteten Austausch und einem Verknüpfen von Wissensund Erfahrungsbeständen besteht, was es ermöglicht, gemeinsame Problemlösungen aufeinander $\mathrm{zu}$ beziehen und diese konsenshaft $\mathrm{zu}$ kollegialem Wissen zusammenzuführen. Den drei Kooperationsformen liegen Annahmen aus der Organisationspsychologie sowie zu Kernbedingungen der Kooperation in Schulen zugrunde (Tabelle 4.1).

Tabelle 4.1 Kernbedingungen der Kooperationsformen „Austausch“, ,,arbeitsteilige Kooperation“ und „Ko-Konstruktion“ nach Gräsel et al. (2006a)

\begin{tabular}{l|l|l|l}
\hline Kernbedingungen & Austausch & $\begin{array}{l}\text { Arbeitsteilige } \\
\text { Kooperation }\end{array}$ & Ko-Konstruktion \\
\hline Zielinterdependenz & Nicht erforderlich & Erforderlich & Erforderlich \\
\hline Vertrauen & Erforderlich & Erforderlich & Besonders wichtig \\
\hline Autonomie & Sehr hoch & Hoch & Eingeschränkt \\
\hline Gemeinsame Arbeit & Nicht vorhanden & $\begin{array}{l}\text { Verständigung über } \\
\text { Arbeitsteilung }\end{array}$ & Vorhanden \\
\hline Kooperationsaufwand & Gering & Mittel & Hoch \\
\hline Funktion & $\begin{array}{l}\text { Informations und } \\
\text { Materialaustausch }\end{array}$ & $\begin{array}{l}\text { Effizienzsteigerung } \\
\text { durch } \\
\text { Beiträge mehrerer } \\
\text { Personen }\end{array}$ & $\begin{array}{l}\text { Gemeinsames } \\
\text { Aufgaben und } \\
\text { Problemlösen }\end{array}$ \\
\hline
\end{tabular}


Bei der Ko-Konstruktion sind Zielinterdependenzen erforderlich, Vertrauen ist besonders wichtig und die Handlungsautonomie am stärksten eingeschränkt. Die gemeinsame Arbeit an gemeinsamen Entwicklungsgegenständen steht im Zentrum und der Kooperationsaufwand ist am höchsten. Ko-Konstruktion ist somit die voraussetzungsvollste, anspruchsvollste und umfangreichste der drei beschriebenen Kooperationsformen (Gräsel et al., 2006a). Allerdings bilden diese keine qualitative Reihenfolge im Sinne von ,Je intensiver desto besser“ ab. Jede Kooperationsform hat vielmehr eine spezifische Funktion und je nach Situation ist eine andere zweckmäßiger und zielführender (Fussangel \& Gräsel, 2011; Gräsel et al., 2006a).

Die Kooperationsformen wurden in Folgestudien und auch von anderen Forschungsgruppen rezipiert und weiterentwickelt. So unterscheiden Fussangel et al. (2010) sowie Soltau und Mienert (2013) zwischen Austausch, schülerbezogenem Austausch, Synchronisation und Ko-Konstruktion. Keller-Schneider und Albisser (2012, 2013) wiederum differenzieren ko-konstruktive Kooperation weiter in (1) gemeinsame Unterrichtsplanung mit gemeinsamer Vereinbarung von Lernzielen, Vorgehensweisen und Lernzielüberprüfung, (2) Diskussion von pädagogischen Fragen, wobei sich die Gesprächspartnerinnen und Gesprächspartner mit verschiedenen Perspektiven auseinandersetzen sowie Erwartungen klären, und (3) gemeinsam getragene Verantwortung in der Unterrichtsdurchführung. In einem Modell von Lehrpersonenkooperation von Vogt, Kunz Heim und Zumwald (2016) sind Austausch, Synchronisation, arbeitsteilige Kooperation, Ko-Konstruktion und Reflexion aufgeführt.

\section{Häufigkeiten ko-konstruktiver Kooperation}

Studien im deutschsprachigen Raum zur Häufigkeit von Ko-Konstruktion zeigen, dass diese in der überwiegenden Zahl der untersuchten Fälle selten bis gar nicht vorkommt (Buddeberg et al., 2014; Fussangel et al., 2010; Gräsel et al., 2006a; Helmke \& Jäger, 2002; Holtappels, 1999; Killus \& Gottmann, 2012; Kunz Heim, Arnold, Eschelmüller \& Achermann, 2013; Massenkeil \& Rothland, 2016; Pröbstel, 2008; Soltau \& Mienert, 2013; Stommel, Hildebrandt, Senn \& Widmer, 2014; Zentrum für Bildungsforschung und Lehrerbildung, 2009). Zudem lassen sich Unterschiede zwischen den Schulformen erkennen: Je höher die Schulform, desto weniger kooperieren die Lehrpersonen miteinander (Ahlgrimm, 2010; Bauer, 2008; Helmke \& Jäger, 2002; Klieme et al., 2008; Lohre, Kober, Madelung, Schnoor \& Weisker, 2006; Maag Merki et al., 2010; Soltau, 2011; Steinert et al., 2006; Tillmann, 2011).

Bemerkenswert ist, dass neuere Studien im Kontext heterogener Lerngruppen deutlich höhere Anteile ko-konstruktiver Kooperation aufweisen. Dies konnte zum 
Beispiel in der wissenschaftlichen Begleitforschung zur Einführung von Gemeinschaftsschulen in Baden-Württemberg (Bennemann \& Schönknecht, 2016) oder in Untersuchungen zur integrativen Schulform in der Schweiz und zum damit einhergehenden Team-Teaching zwischen Regellehrperson und Fachpersonen schulischer Sonderpädagogik (Maag Merki et al., 2010; Reusser et al., 2013) nachgewiesen werden. So zeigte sich beispielsweise in der Studie von Maag Merki et al. (2010), dass 55.6 Prozent der befragten Lehrpersonen angegeben hatten, Kooperationsinhalte gemeinsam reflexiv zu bearbeiten. 28.0 Prozent bearbeiteten Kooperationsinhalte in arbeitsteiliger Weise und 16.4 Prozent beschränkten sich vor allem auf den Austausch von Material und Wissen. Die Analyse quantitativer Daten von Henrich, Baumann und Studer (2012) zur Kooperation von Schweizer Lehrpersonen im integrativen Unterrichtssetting konnte belegen, dass 51.2 Prozent der untersuchten Lehrpersonen in den Team-TeachingTeams ko-konstruktiv kooperieren. Allerdings beruhen diese Ergebnisse alle auf Selbstberichten von Lehrpersonen, die zwar Anhaltspunkte für mögliche Interpretationsansätze zu liefern vermögen, aber keine konkreten Schlüsse auf die tatsächlich stattfindenden Kooperationspraktiken zulassen. Um die konkrete Handlungsebene untersuchen zu können, eignen sich Videostudien wie beispielsweise jene von Hildebrandt et al. (2017). Sie videografierten Sitzungen von Team-Teaching-Teams und analysierten diese u. a. hinsichtlich der Häufigkeit ko-konstruktiver Kooperation. Die Analysen zeigten, dass die Teams unterschiedlich häufig ko-konstruktiv kooperieren. Zudem konnten bei allen Teams, auch bei jenen mit höherem Anteil ko-konstruktiver Kooperation, kaum tiefgreifende Reflexionen und intensive Phasen der gegenseitigen Bezugnahme von Wissensund Erfahrungsbeständen beobachtet werden.

\section{Wirkungen und Voraussetzungen ko-konstruktiver Kooperation}

Wie in den vorhergehenden Abschnitten dargelegt wurde, arbeiten Lehrpersonen in Schulentwicklungsprozessen häufiger miteinander zusammen, allerdings nicht immer zwingend ko-konstruktiv (Feldhoff, Kanders \& Rolff, 2008). Jedoch scheinen gerade der Austausch und das Verknüpfen von Wissens- und Erfahrungsbeständen wichtig zu sein, um Probleme in der Schul- und Unterrichtsentwicklung gemeinsam zu lösen (u. a. Gräsel et al., 2006a). Kooperationsstrukturen, in denen ko-konstruktive und unterrichtsbezogene Kooperation ermöglicht wird, gelten deshalb als höchst relevant für erfolgreiche Entwicklungstätigkeiten, die sich wiederum auf den Lernerfolg von Schülerinnen und Schülern auswirken. Als zweckdienlich haben sich diesbezüglich zum Beispiel Unterrichtshospitation und gemeinsame Unterrichtsplanung erwiesen (Helmke, 2017; Terhart \& Klieme, 2006) oder Fachspezifisches-Pädagogisches Coaching (Staub, 2004; West 
\& Staub, 2008) und Kollegiales Unterrichtscoaching (Kreis \& Staub, 2017). Gerade wenn Kooperation auf Unterrichtsthemen bezogen ist, wird ihr eine hohe Wirksamkeit zugesprochen (Krammer, Schnetzler, Pauli, Ratzka \& Lipowsky, 2009; Kreis, Lügstenmann \& Staub, 2008; Kullmann, 2010; Maag Merki et al., 2010).

(Angehende) Lehrpersonen, die in solchen Strukturen Unterricht gemeinsam reflektieren und weiterentwickeln, bilden professionelle Lerngemeinschaften (u. a. Bonsen, 2010; Bonsen \& Rolff, 2006). In professionellen Lerngemeinschaften werden beispielsweise qualitätsvolle Unterrichtseinheiten und -materialien gemeinsam entwickelt, in einem reflexiven Dialog wird offen über Chancen und Herausforderungen des eigenen unterrichtlichen Handelns gesprochen, Lernprozesse der Schülerinnen und Schüler werden fokussiert und es wird gemeinsame Verantwortung für Schulentwicklung übernommen (Bonsen, 2010; Stoll, Bolam, McMahon, Wallace \& Thomas, 2006). Ziel ist es, Professionalisierungsprozesse der Lehrpersonen hinsichtlich des unterrichtlichen Handelns und des Lernens von Schülerinnen und Schülern zu initiieren (Rolff, 2014; Stoll et al., 2006), was insbesondere durch zielführendes Diskutieren und Reflektieren erreicht wird (Bonsen, 2010; Fussangel \& Gräsel, 2011; Gräsel et al., 2006a; A. A. Huber, 2010; S. G. Huber, Ahlgrimm \& Hader-Popp, 2012; Kelchtermans, 2006; KellerSchneider \& Albisser, 2013; Klinger, 2013; Maag Merki et al., 2010; Pröbstel, 2008; Zala-Mezö \& Hameyer, 2016). Kooperation im Sinne von professionellen Lerngemeinschaften hat zudem positive Auswirkungen auf die Selbstständigkeit und die Lernmotivation der Schülerinnen und Schüler sowie auf das Unterrichtsklima im Klassenzimmer (C. Arnold, Bauer, Kunz Heim \& Eschelmüller, 2011). Weitere Studien und Meta-Analysen konnten auch positive direkte und indirekte Wirkungen auf die Lernleistungen nachweisen (Bryk, Sebring, Allensworth, Luppescu \& Easton, 2010; Hord, 1997; Lipowsky, 2014; Lomos, Hofman \& Bosker, 2011, 2012).

Damit Ko-Konstruktion gelingt, sind wie in Abschnitt 4.5.2 bereits festgehalten unterstützende organisationale Rahmenbedingungen - wie etwa vorhandene Zeit für Zusammenarbeit und geeignete Arbeitsräume - notwendig. Sind diese nicht gegeben oder ist die Terminfindung wegen Teilzeitarbeit oder anderer Verpflichtungen erschwert, kann dies für eine ko-konstruktive Kooperation hinderlich sein (Maag Merki, Holzbrecher, Kotthoff, Ehlert \& Werner, 2009; Reusser et al., 2013; Ulich, 1996; Werner, Maag Merki \& Ehlert, 2009). Auch soziale Spannungen und Konflikte zwischen den Kooperationspartnerinnen und Kooperationspartnern (Achinstein, 2002; Conley, Bas-Isaac \& Scull, 1995; A. Hargreaves, 1994), sich widersprechende individuelle und organisationale Handlungsziele (Altrichter \& 
Salzgeber, 1996; B. Johnson, 2003) oder soziale Vergleiche zwischen den Lehrpersonen, die zu gegenseitiger Abwertung führen (Ahlgrimm, 2012; Ulich, 1996), können den Kooperationsprozess erschweren. Auf der anderen Seite tragen produktive Arbeitsbeziehungen, Respekt und Vertrauen sowie ähnliche pädagogische Grundhaltungen zum Gelingen ko-konstruktiver Kooperation bei (Gräsel et al., 2006a; B. Johnson, 2003; Reusser et al., 2013; Vangrieken, Meredith, Packer \& Kyndt, 2017). Intensive Kooperation kann das Wohlbefinden und die Motivation steigern; die Lehrpersonen fühlen sich in einer Gruppe angenommen und erfahren, dass die ihre Kolleginnen und Kollegen im Unterricht vor ähnlichen Herausforderungen stehen (C. Arnold et al., 2011).

Kooperation in Schulen mit Unterrichtskonzepten personalisierter Gestaltung von Lehr- und Lernprozessen

Die im vorhergehenden Abschnitt dargelegten Ergebnisse lassen sich auf Schulen mit Konzepten personalisierten Lernens übertragen und ließen sich in den Studien zur Lehrpersonenkooperation in personalisierten Lernumgebungen größtenteils replizieren. Grundsätzlich deuten die Ergebnisse darauf hin, dass in solchen Schulen viel und intensiv kooperiert wird. Zum Beispiel bereiten Lehrpersonen mit und/oder füreinander Grobplanungen vor, erstellen gemeinsam Stoffpläne für alters und/oder leistungsdurchmischte Lerngruppen und erarbeiten Aufgabensammlungen oder entwickeln selbst Lernaufgaben, unterrichten oftmals im Team-Teaching und reflektieren ihren Unterricht gemeinsam. Darüber hinaus besprechen sie herausforderndes Lernverhalten der Schülerinnen und Schüler und unterstützen sich gegenseitig bei anspruchsvollen Elterngesprächen (Galle et al., 2019; Jenkins \& Keefe, 2002; Pane, Steiner, Baird \& Hamilton, 2015; Prain et al., 2015a; Sebba et al., 2007; Stebler et al., eingereicht; Stebler et al., 2017, 2018; Waldrip et al., 2016; Yonezawa et al., 2012). Diese Auflistung verweist auf eine unterrichtsbezogene Kooperation, die vermehrt auf individuelle Lernprozesse der Schülerinnen und Schüler und deren Ermöglichen ausgerichtet ist: „all these schools create regular, frequent opportunities for teachers to collaborate with one another for the benefit of their students" (Cervone \& Cushman, 2012, S. 40). Des Weiteren lässt sich ko-konstruktive Kooperation in Tätigkeiten der Schul- und Unterrichtsentwicklung beobachten. So erarbeiten sich die Lehrpersonen einerseits ein gemeinsames Wissen und Vorstellungen in Bezug darauf, wie sich personalisiertes Lernen konkret in ihrer Schule charakterisieren lässt, und sie legen fest, wie sie ihre Rolle als Lehrperson im neuen Unterrichtskonzept gestalten. Andererseits bearbeiten sie gemeinsam Herausforderungen und Probleme bei der Einführung und Weiterentwicklung der Konzepte personalisierten Lernens (Galle et al., 2019; Prain et al., 2015b; Stebler et al., eingereicht). Zudem 
werden in einigen Schulen systematische Unterrichtsbesuche und Mentorenprogramme eingeführt, bei denen sich die Lehrpersonen über ihre Berufstätigkeiten in den personalisierten Lernumgebungen austauschen und neue Lehrpersonen in das Unterrichtskonzept einführen (Cervone \& Cushman, 2012).

\subsubsection{Professionalisierung des Schulpersonals und Personalrekrutierung}

Damit Lehrpersonen Entwicklungsprozesse planen, steuern und Veränderungen nachhaltig im Unterricht implementieren können, benötigen sie Wissen, Fähigkeiten und Fertigkeiten (Terhart, 2016; Zlatkin-Troitschanskaia \& Förster, 2009). Anhand zweier Modelle wird nachfolgend dargelegt, welche Kompetenzbereiche bei einer unterrichtszentrierten Schulentwicklung in Richtung personalisierten Lernens wichtig sein können. Anschließend werden Möglichkeiten der Professionalisierung des Schulpersonals aufgezeigt. Im letzten Unterabschnitt wird die gezielte Personalrekrutierung von qualifizierten und motivierten Lehrpersonen als eine weitere Form der Professionalisierung des Kollegiums beschrieben.

\section{Lehrpersonenbezogene Kompetenzmodelle}

Ein im deutschsprachigen Bildungsraum oft zitiertes Modell professioneller Handlungskompetenzen stammt von Baumert und Kunter (2006). Sie unterscheiden motivationale Orientierungen, selbstregulative Fähigkeiten, Überzeugungen und Werthaltungen sowie Professionswissen. Letzteres untergliedern der Autor und die Autorin in pädagogisches Wissen, Fachwissen, fachdidaktisches Wissen, Organisationswissen und Beratungswissen. Solche unterrichtsbezogenen Kompetenzen sind in einer unterrichtszentrierten Schulentwicklung in Richtung personalisierten Lernens relevant, weil sie, wie auch ein Entwicklungsprozess, auf den Unterricht und auf den Lernerfolg von Schülerinnen und Schülern ausgerichtet sind. Lehrpersonen sollten über genügend Professionswissen verfügen, um Entwicklungsbedarf sowohl auf der Oberflächenstruktur als auch auf der Tiefenstruktur des Unterrichts zu erkennen und adäquate Veränderungsprozesse zu initiieren (siehe Abschnitt 4.5.1.3). Günstige Motivationslagen, Überzeugungen und Werthaltungen sowie selbstregulative Fähigkeiten unterstützen die Entwicklungstätigkeiten.

Ein weiteres Kompetenzmodell, das von A. Hargreaves und Fullan (2012) entwickelt wurde, stellt das Konzept des professional capital als eine Funktion aus human, social und decisional capital in den Mittelpunkt. Das professional capital ist umso grösser, je ausgeprägter die Kompetenzen in den drei 
Teilbereichen sind. Unter human capital verstehen die Autoren das Wissen, die Fähigkeiten und die Fertigkeiten, zu unterrichten. Konkret sind damit beispielsweise fachliches und fachdidaktisches Wissen sowie Kenntnisse über die Lernvoraussetzungen der Schülerinnen und Schüler gemeint. Social capital hingegen manifestiert sich in der Quantität und der Qualität von Interaktionen und sozialen Beziehungen mit Schülerinnen und Schülern, anderen Lehrpersonen und weiteren Personen aus dem Schulbereich. Zwar enthalten beide Kapitalformen Elemente des Kompetenzbereichs „Professionswissen“ von Baumert und Kunter (2006), jedoch beinhaltet Letzteres darüber hinaus eine Kompetenz, die für unterrichtszentrierte Schulentwicklung in Richtung personalisierten Lernens unerlässlich ist und im Modell von Baumert und Kunter (2006) nicht aufgeführt wird: die Kooperation zwischen Lehrpersonen. Es wurde bereits ausgeführt (siehe Abschnitt 4.5.3), dass insbesondere eine unterrichtsbezogene und ko-konstruktive Kooperation bedeutsam für einen erfolgreichen Entwicklungsprozess und eine erfolgreiche Umsetzung eines Konzepts personalisierten Lernens ist. Die Fähigkeit zur aktiven Teilnahme an einer solchen Kooperation, bei der Lehrpersonen ihr eigenes unterrichtliches Handeln offen mit anderen Lehrpersonen reflektieren, Wissens- und Erfahrungsbestände miteinander verknüpfen und eine vertrauensund respektvolle Gesprächskultur pflegen, ist daher ebenfalls als professionelle Kompetenz beschreibbar. Die letzte Kapitalform ist das decisional capital. Hierunter verstehen die Autoren die Fähigkeit der Lehrperson, Entscheidungen zu treffen. Dies gilt besonders für die Arbeit im Klassenzimmer, in der komplexe Situationen in sehr kurzer Zeit beobachtet und analysiert werden müssen und situativ eine Handlungsentscheidung zu treffen ist (Fullan, 2016; A. Hargreaves \& Fullan, 2012).

\section{Schulinterne und -externe Fort- und Weiterbildungen}

Kompetenzen können Lehrpersonen in schulinternen und -externen Fort- und Weiterbildungen erwerben und ausbauen. In der Schweiz sind Weiterbildungen ein fester Bestandteil des Berufsauftrags. Zum Beispiel verfügen Schulen in der Stadt Zürich in einem Schuljahr über fünf Tage, an denen Schulleitende und Lehrpersonen eigenständig Weiterbildungen planen und umsetzen können. Allerdings zeigen Forschungsergebnisse zu internen Weiterbildungen an Berufsschulen im Kanton Zürich, dass die Themenauswahl kaum langfristig geplant ist und kaum systematisch erfolgt (Fischer, 2016). Studienergebnisse aus Deutschland wiederum kamen zum Schluss, dass gezielte Fortbildungen in und außerhalb der Einzelschule von Lehrpersonen in Entwicklungsprozessen zwar erwünscht sind, aber selten angeboten oder genutzt werden (Bastian, 2007; Bonsen, 2010; Seitz, 
2008). So lässt sich den Ergebnissen der Begleitforschung zum Projekt „Selbstständige Schule" in Deutschland zwar entnehmen, dass die Lehrpersonen und die Schulleitenden Weiterbildungsangebote für ihre Schule gezielt auswählten. Gleichwohl ließen sich in den untersuchten Schulen kaum Weiterbildungskonzepte finden, die systematisch mit den Entwicklungsprozessen verknüpft waren. Dennoch konnten kleinere Effekte auf der Oberflächenstruktur des Unterrichts festgestellt werden, die darin bestanden, dass die Lehrpersonen Unterrichtsmethoden variabler einsetzten. Wirkungen hinsichtlich verbesserter Prozessqualitäten des Lernens von Schülerinnen und Schülern konnten hingegen nicht nachgewiesen werden (Holtappels \& Voss, 2008a; Klemm \& Meetz, 2008).

Angesichts dieser Befunde stellt sich die Frage, wie nachhaltig ein eher unsystematisch gestaltetes Weiterbildungsangebot und dessen lediglich partielle Nutzung für Entwicklungsprozesse sind. Soll eine Fort- und Weiterbildung über die Kompetenzerweiterung der Lehrpersonen auf die Prozessqualitäten schulischen Lernens wirken, bedarf es eines auf die Bedürfnisse der Lehrpersonen angepassten Weiterbildungsangebots (Göb, 2017). Lipowsky (2014) trug aus nationaler und internationaler Forschungsliteratur Merkmale lernwirksamer Weiterbildungen zusammen (u. a. auch Lipowsky, 2017; Lipowsky \& Rzejak, 2015a, 2015b). Lernwirksam sind gemäß seinen Ergebnissen Merkmale, welche direkte Effekte auf erweitertes unterrichtliches Handeln der Lehrpersonen und auf verbesserte Unterrichtsqualität aufweisen sowie indirekte Effekte auf kognitive und affektiv-motivationale Veränderungen der Lernenden zeigen (Lipowsky, 2010):

- verschränkte und aufeinander aufbauende Input-, Erprobungs- und Reflexions/Feedbackphasen,

- Feedback und Coaching, um das Lehrpersonenhandeln und den Unterricht in der von der Fortbildung ,intendierten Weise weiterzuentwickeln“ (Lipowsky \& Rzejak, 2015b, S. 146),

- eigene Handlungswirksamkeit erfahren, indem Lehrpersonen eine Verbindung zwischen dem eigenen Handeln und dem Lernen der Schülerinnen und Schüler schaffen,

- Anknüpfen an und Weiterentwickeln von fachlichem und fachdidaktischem Wissen sowie von kognitiven Voraussetzungen der Teilnehmenden,

- verbessertes diagnostisches Wissen über fachbezogene Lern- und Verstehensprozesse der Schülerinnen und Schüler und deren Lernergebnisse sowie ein darauf bezogenes Erstellen adaptiver Lernangebote,

- Einbezug von Ergebnissen allgemeindidaktischer (z. B. Qualitätsmerkmale guten Unterrichts) und fachdidaktischer (z. B. Förderung von Lesestrategien) Unterrichtsforschung, 
- Wissenschaftlerinnen und Wissenschaftler sind an der Planung wirkungsvoller Weiterbildung beteiligt und bringen ihre Expertise ein,

- Weiterbildungsdauer von mehr als 30 Stunden,

- inhaltliche Relevanz der Weiterbildung für Teilnehmende und

- Zusammenarbeit der Teilnehmenden in professionellen Lerngemeinschaften (Lipowsky, 2014, 2017; Lipowsky \& Rzejak, 2015a, 2015b).

Im Sinne eines Angebot-Nutzungs-Modells ist die Wirkung von Weiterbildungen jedoch nicht nur von der Qualität des Angebots abhängig, sondern ebenso vom Nutzungsverhalten der teilnehmenden Lehrpersonen, wozu zum Beispiel Akzeptanz und wahrgenommene Relevanz der Weiterbildung oder lernförderliche Einstellungen gehören (Lipowsky, 2010; Stommel, Hildebrandt, Senn \& Widmer, 2015).

Potenziale von Professionalisierungsprozessen in unterrichtszentrierter Schulentwicklung in Richtung personalisierten Lernens

Mit dem letzten Punkt ihrer Zusammenstellung von Merkmalen effektiver Weiterbildungen - der Zusammenarbeit der Teilnehmenden in professionellen Lerngemeinschaften - weisen Lipowsky und sein Team darauf hin, dass in professionellen Lerngemeinschaften durch intensive Zusammenarbeit Professionalisierungsprozesse angeregt werden können. Bereits im Kapitel zur Entwicklung der Kooperation von Lehrpersonen (siehe Abschnitt 4.5.3) wurde erstens dargelegt, dass ko-konstruktive unterrichtsbezogene Kooperation einen beruflichen Kompetenzaufbau unterstützt, und zweitens festgehalten, dass in einer unterrichtszentrierten Schulentwicklung in Richtung personalisierten Lernens nicht nur im Zusammenhang mit Entwicklungstätigkeiten ko-konstruktiv und unterrichtsbezogen zusammengearbeitet wird, sondern auch in der alltäglichen Unterrichtsarbeit.

Das lässt den Schluss zu, dass in Konzepten personalisierten Lernens viele Möglichkeiten für Professionalisierungsprozesse vorzufinden sind. Allerdings ist ko-konstruktive unterrichtsbezogene Kooperation sehr voraussetzungsvoll. Studien zu förderlichen und hinderlichen Bedingungen ko-konstruktiver Kooperation (siehe Abschnitt 4.5.3) geben diesbezüglich wichtige Hinweise. Eine Untersuchung an über 23 Schulen mit Konzepten personalisierten Lernens der Forschungsgruppe RAND Corporation (2014) beispielsweise zeigt auf, dass zwar 91 Prozent der Lehrpersonen stark oder eher damit übereinstimmten, dass die beruflichen Tätigkeiten mit anderen Lehrpersonen in personalisierten Lernumgebungen sie dazu ermutigen würden, ihre eigene Unterrichtspraxis zu reflektieren und zu verbessern. Vor allem eine Zusammenarbeit hinsichtlich der individuellen Förderung von Schülerinnen und Schülern wurde für die eigene Kompetenzentwicklung 
als positiv bewertet. Zugleich gaben 50 Prozent der Lehrpersonen jedoch an, dass solch kollegiale Kooperationen zu viel Zeit in Anspruch nähmen. Zudem berichteten diese Lehrpersonen, dass in Sitzungen und Besprechungen zu viele Themen angesprochen und die Diskussionen schnell unübersichtlich würden.

\section{Personalrekrutierung}

Neben Fort- und Weiterbildungen sowie Professionalisierung durch kokonstruktive unterrichtsbezogene Kooperation besteht eine weitere Strategie, um qualifiziertes Personal für eine unterrichtszentrierte Schulentwicklung in Richtung personalisierten Lernens zu erhalten, in der gezielten Rekrutierung von kompetenten und motivierten Lehrpersonen. In Deutschland werden Lehrpersonen häufig einer Schule zugeteilt, unabhängig davon, ob die Wünsche der betreffenden Lehrperson mit dem Schulprofil übereinstimmen oder nicht (Terhart, 2016). Formen erweiterter Schulautonomie, bei der Personalangelegenheiten vermehrt von Schulleitenden verantwortet werden, werden zwar sowohl von Schulleitenden als auch von Lehrpersonen positiv bewertet, sind aber noch nicht weit verbreitet (Klemm \& Meetz, 2008). In der Schweiz hat sich dagegen seit der Einführung von Schulleitungen in den 1990er-Jahren in vielen Kantonen das Bewerbungsverfahren etabliert (Maag Merki, 2011). Eine neue Stelle als Lehrperson wird offiziell ausgeschrieben. Dadurch hat die Schulleitung die Möglichkeit, auf das Schulprofil hinzuweisen und in Übereinstimmung damit Lehrpersonen einzustellen, die sich für dieses Schulprofil interessieren.

\subsubsection{Entwicklung eines gemeinschaftlichen Schullebens}

Die letzte der fünf Entwicklungsdimensionen des uSpL-Modells ist die Entwicklung eines gemeinschaftlichen Schullebens. Sie umfasst die gemeinsame und belebende Gestaltung von über den Unterricht hinausgehenden Schulaktivitäten (Klafki, 1985/2007; Saalfrank, 2016). Ein anregendes Schulleben kann nachweislich positive Auswirkungen auf das soziale Schulklima haben (Holtappels \& Voss, 2008b; Saalfrank, 2016). Merkmale der Gestaltung des Schullebens sind (1) die Schaffung von Lerngelegenheiten außerhalb des Unterrichts mit Musik-, Theater- oder Sportprojekten, Wandertagen, Exkursionen, Ausstellungen, Feiern, Tagen der offenen Tür etc. (2) Diese Lerngelegenheiten umfassen eine didaktische Komponente, etwa wenn mit Schülerinnen und Schülern im Hinblick auf die Aufführung eines Theaterstücks mit verschiedenen Lehr- und Lernmethoden der Theaterpädagogik geprobt wird. Hinzu kommt in diesem Zusammenhang eine erzieherische Komponente. Bei den Theaterproben lernen die Schülerinnen und 
Schüler, die Bedürfnisse und Emotionen ihrer Mitschülerinnen und Mitschüler zu respektieren und darauf einzugehen. (3) Für das Schulleben ist des Weiteren eine unterstützende Infrastruktur nötig. Zum Beispiel braucht es für Theaterproben entsprechende Räumlichkeiten und Requisiten. Auch eine Bibliothek, separate Lernräume für Projektarbeit oder die Unterstützung durch die Eltern gelten als förderliche Rahmenbedingungen. (4) Die Partizipation der Schülerinnen und Schüler in Projekten und am Schulleben ist zentral. Die Schülerinnen und Schüler agieren als Schauspielerinnen und Schauspieler im Theaterstück oder sie können durch Klassen- und Schülerparlamente das Schulleben zu einem bestimmten Grad mitbestimmen.

In Schulen mit Konzepten personalisierten Lernens ist die klare Trennung zwischen Unterricht und über den Unterricht hinausgehenden Aktivitäten häufig fließend. So können Lernthemen aus den geführten Unterrichtsphasen in offeneren Unterrichtsphasen weitergeführt und je nach persönlichem Interesse beispielsweise in Projektarbeiten vertieft werden, welche die Schülerinnen und Schüler zum Beispiel an einem Tag der offenen Tür präsentieren können (u. a. Müller, 2014). Das gemeinschaftliche Schulleben lässt sich zum Teil in der fünften Dimension personalisierten Lernens (Stebler et al., 2018) verorten (siehe Abschnitt 3.2), die sich dadurch kennzeichnet, dass die Lerngemeinschaft bildend und unterstützend wirkt. Diese Lerngemeinschaft setzt sich aus den Lehrpersonen und den Schülerinnen und Schülern zusammen und wird durch die verschiedenen Lernbeziehungen zwischen den Beteiligten geprägt.

Aber auch die Lehrpersonen tragen mit einer intensiven Kooperation im Team zu einer bildenden und unterstützenden Gemeinschaft bei, indem sie gemeinsam das jeweilige Konzept personalisierten Lernens umsetzen und weiterentwickeln (siehe Abschnitt 4.5.3). Ergebnisse des perLen-Forschungsprojekts deuten diesbezüglich auf ein verstärktes gemeinschaftliches „Unterwegssein“ hin: So unterschieden sich die sehr stark personalisierten Schulen von moderat personalisierten Schulen (Gruppendefinition siehe Abschnitt 4.5.1.4) durch häufigeres gemeinsames Unterrichten im Team-Teaching und eine produktiver eingeschätzte Zusammenarbeit zwischen den Schülerinnen und Schülern (Stebler et al., 2018).

\subsection{Entwicklungsprozess in einer unterrichtszentrierten Schulentwicklung in Richtung personalisierten Lernens}

Nachdem mit den aufgeführten Auslösern einer unterrichtszentrierten Schulentwicklung in Richtung personalisierten Lernens (siehe Abschnitt 4.4) das Warum 
einer solchen Entwicklung beschrieben wurde, bezogen sich die im vorhergehenden Kapitel umrissenen fünf Tätigkeitsfelder (Entwicklungsdimensionen) auf das Was, das heißt auf die Frage, welche Gegenstände und Inhalte im Entwicklungsprozess bearbeitet werden. In diesem Kapitel wird nun das Wie bearbeitet: Wie ist ein Entwicklungsprozess zu beschreiben, welche Phasen umfasst er und welche Handlungsdynamiken liegen ihm zugrunde?

Ein Entwicklungsprozess lässt sich durch drei Merkmale charakterisieren (Abbildung 4.15): (1) Die involvierten Personen können einen Entwicklungsprozess steuern, vorantreiben und auch stoppen (Fend, 2008b; Schlee, 2014). (2) Ein Entwicklungsprozess ist komplex, weil individuelle, institutionelle, inhaltliche, soziale und kulturelle Aspekte ineinandergreifen (Haenisch, 2016; Meyer, 2015; Rolff, 2013). (3) Aus einer soziokonstruktivistischen Perspektive (Vygotskij, 1934/2002) kann ein Entwicklungsprozess als kooperativer Aushandlungsprozess um bestmögliche Lernumgebungen für Schülerinnen und Schüler verstanden werden, der in Abhängigkeit vom sozialen Kontext und von den gegebenen Rahmenbedingungen stattfindet. Die Mitwirkenden erarbeiten sich auf der Grundlage der eigenen Vorerfahrungen, der kognitiven Voraussetzungen und der Interaktionen mit anderen Personen ein Verständnis von personalisiertem Lernen und davon, wie dieses in der alltäglichen Unterrichtsarbeit umgesetzt werden sollte. In kollegialer Kooperation werden die Auffassungen abgeglichen und zu gemeinsamen Vorstellungen verknüpft, die anschließend als Ausgangspunkt für die Planung und Umsetzung möglicher konkreter Entwicklungstätigkeiten dienen können. Aus dieser Perspektive kann ein Entwicklungsprozess somit als ein dynamisches Konstrukt beschrieben werden, welches sich durch Konstruktions- und Ko-Konstruktionsleistungen der Kooperationspartnerinnen und Kooperationspartner permanent verändert (Kiper, 2013; Rahm, 2009) und sich durch die drei Phasen der Initiation, der Implementation und der Institutionalisierung (Abbildung 4.15; Giaquinta, 1973) sowie die Wechselwirkung zwischen Struktur und Handlung (Giddens, 1988) charakterisieren lässt.

\section{Initiation, Implementation, Institutionalisierung}

In der von Giaquinta (1973) auf der Grundlage eines Forschungsreviews zu Innovationsprozessen (organizational change) beschriebenen ersten Phase der Initiation (initiation) eruieren die am Entwicklungsprozess beteiligten Personen Herausforderungen und Probleme. Mögliche Lösungsansätze werden gesammelt und der passendste ausgewählt. Typische Aktivitäten sind das Analysieren von Problemen, das Bilden von Konsens über Entwicklungsziele, die Entwicklung von Visionen, das Einrichten einer Prozesssteuerung, die Erarbeitung eines Projektplans und von Konzepten sowie das Informieren und das Überzeugen des 


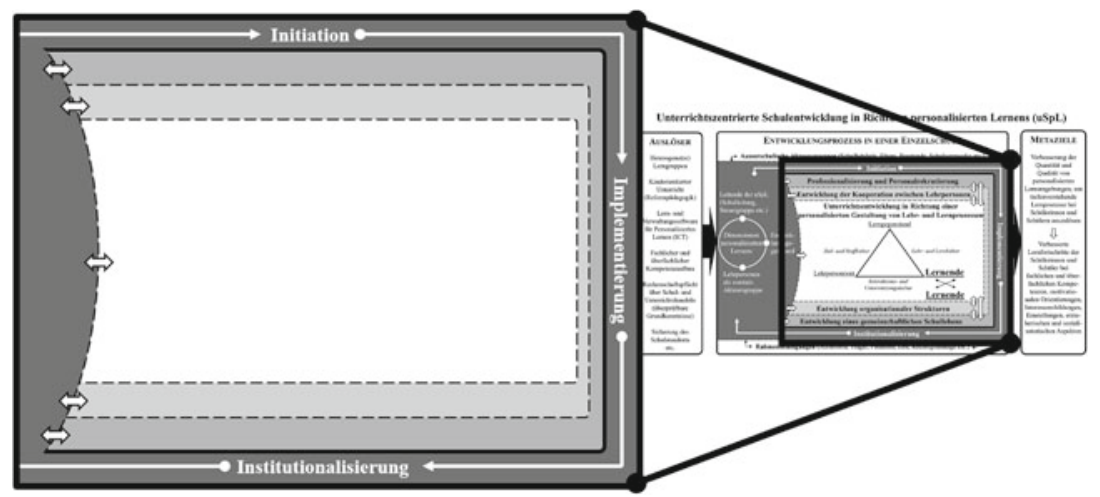

Abbildung 4.15 Phasen „Initiation“, „Implementation“ und „Institutionalisierung“ (Giaquinta, 1973) im uSpL-Modell (eigene Darstellung)

Kollegiums (Hameyer, 2005; Holtappels, 2014). In der Implementationsphase (implementation) wird der Entwicklungsgegenstand in den Schul- und Unterrichtsalltag eingeführt, Handlungsabläufe werden an den Neuerungen ausgerichtet und Haltungen und Einstellungen gegebenenfalls angepasst (Giaquinta, 1973). Der Projektplan und die entwickelten Konzepte werden in der Praxis erprobt. Das Kollegium wird aktiv miteinbezogen und erste Evaluationen und Optimierungen der Konzeption und der Handlungsabläufe finden statt (Hameyer, 2005; Holtappels, 2014). Unter dem Begriff der Institutionalisierung (incorporation) sind das Stabilisieren und das Automatisieren der neuen Handlungsabläufe zu verstehen sowie das Festigen der angepassten Haltungen und Einstellungen (Giaquinta, 1973). Qualität und Nachhaltigkeit des Entwicklungsprozesses werden überprüft und gesichert (Hameyer, 2005; Holtappels, 2014).

Diese drei analytisch separierten Phasen sind in der Praxis weniger getrennt voneinander zu denken, sondern vielmehr als sich überlappende Phasen aufzufassen, die auch parallel verlaufen können (Demmer-Dieckmann, 2005; Holtappels, 2014; Röhrich, 2013). Des Weiteren können Schulentwicklung im Allgemeinen und unterrichtszentrierte Schulentwicklung in Richtung personalisierten Lernens im Besonderen eher als fortlaufender Prozess denn als ein fertiges Produkt verstanden werden. Es gibt fast immer Aspekte und Bereiche im Unterricht und in der Schule, die es anzupassen und zu optimieren gilt (Demmer-Dieckmann, 2005; D. Hargreaves, 2006b; Helmke, 2017; Röhrich, 2008). 
Wechselwirkung zwischen Struktur und Handeln

Giddens (1988) beschrieb in seinem Modell die Wechselwirkungen zwischen Strukturmomenten und situativem Handeln (Abbildung 4.16).

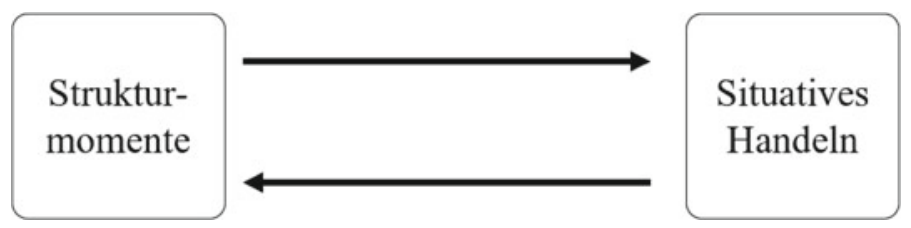

Abbildung 4.16 Wechselwirkungen zwischen Strukturmomenten und situativem Handeln (Giddens, 1988)

Schul- und Unterrichtsstrukturen geben das Handeln von Akteurinnen und Akteuren vor. Unter "Strukturen“ sind in diesem Zusammenhang institutionelle Regelsysteme, beispielsweise der Stundenplan oder Pausenhofregeln, oder institutionalisierte Abläufe von Lehr- und Lernformen zu verstehen (Fend, 2008a, S. 169). Diese Schul- und Unterrichtsstrukturen können durch situatives Handeln verändert werden, zum Beispiel dann, wenn die Lehrpersonen es als erforderlich erachten, wegen des veränderten Pausenhofverhaltens der Schülerinnen und Schüler die Pausenhofregeln anzupassen. In diesem Sinne sind auch die Entwicklungsgegenstände strukturgebend (Abbildung 4.17). Denn die Festlegung von neuen Entwicklungsgegenständen, das heißt desjenigen, was als Nächstes entwickelt werden soll, führt zu einer Ausrichtung der Handlungen auf ebendiesen Gegenstand. Auch bei der unterrichtszentrierten Schulentwicklung in Richtung personalisierten Lernens setzen Leitende wie auch die Lehrpersonen als zentrale Entwicklungshandelnde Entwicklungsziele und erstellen Handlungspläne, um den Entwicklungsgegenstand umzusetzen. Herausforderungen in der Umsetzung dazu führen, dass der Entwicklungsgegenstand erneut angepasst oder ein neuer gewählt wird.

Weitere Strukturmomente wurden bereits im Kapitel zur Entwicklung organisationaler Strukturen erläutert (siehe Abschnitt 4.5.2): Lernräume, Stundenpläne, die Zusammensetzung der Lerngruppen und die Team- und Kooperationsstrukturen. Bisher weniger thematisiert wurde demgegenüber das situative Handeln und dessen Determinanten. Situatives Handeln kann mit der klassischen Motivationspsychologie aus dem Zusammenwirken von Handlungssituation und Person erklärt werden (u. a. Vollmeyer \& Rheinberg, 2018). Im Kontext einer unterrichtszentrierten Schulentwicklung in Richtung personalisierten Lernens bezieht 


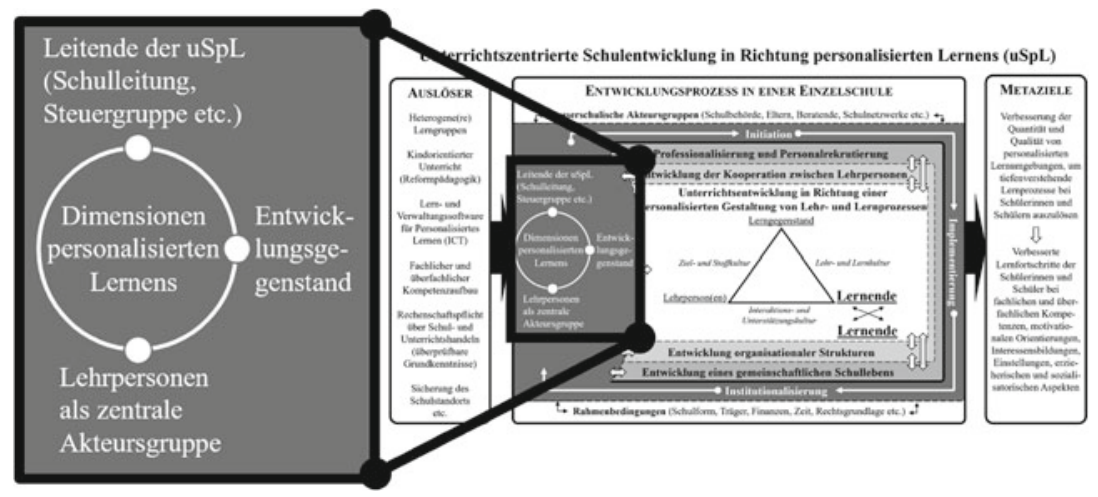

Abbildung 4.17 Wechselwirkungen zwischen Leitenden der unterrichtszentrierten Schulentwicklung in Richtung personalisierten Lernens, Lehrpersonen als zentrale Entwicklungsgruppe und Entwicklungsgegenstand (eigene Darstellung)

sich die Handlungssituation auf das Planen, Umsetzen und Evaluieren des Entwicklungsprozesses. Dieser Prozess wurde im vorhergehenden Abschnitt bereits anhand des Modells von Giaquinta (1973) beschrieben. Unter „Person“ werden in diesem Zusammenhang sowohl stabile, überdauernde Personenmerkmale, sogenannte „Motive“ (u. a. Atkinson, 1957), als auch weniger stabile pädagogisch-psychologische Überzeugungen, Werte und Haltungen (Heckhausen \& Heckhausen, 2010) gefasst:

- Kognitiv- und soziokonstruktivistisches Lehr- und Lernverständnis (u. a. psychologisch-didaktische Grundvorstellungen über Zyklen vollständiger Lernprozesse: PADUA, Aebli, 1983/2006) (siehe Abschnitt 4.5.1.1, Abschnitt 4.5.1.2 und Abschnitt 4.5.1.3),

- Merkmale der Tiefenstruktur des Unterrichts als zentraler Ausgangs- und Zielpunkt eines Entwicklungsprozesses ausgerichtet auf den Lernerfolg von Schülerinnen und Schülern (siehe Abschnitt 4.5.1.3) und

- sozialpsychologische Überzeugungen, Werte und Haltungen: Diese zeigen sich im Verhalten von Schulleitenden, Lehrpersonen und Schülerinnen und Schülern sowie in deren Motivation, den Entwicklungsprozess mitzugestalten und mitzutragen, oder in der Absicht, ihn gegebenenfalls zu behindern (u. a. Schratz \& Steiner-Löffler, 1999). Gerade für eine erfolgreiche ko-konstruktive unterrichtsbezogene Kooperation zwischen Lehrpersonen bezüglich der alltäglichen Unterrichtsarbeit und des Entwicklungsprozesses (siehe Abschnitt 4.5.3) 
sind kooperationsunterstützende Überzeugungen, Werte und Haltungen unabdingbar. Dasselbe gilt für die Zusammenarbeit zwischen Schulleitung und Kollegium (u. a. D. Hargreaves, 2006a) oder zwischen Lehrpersonen und Schülerinnen und Schülern (Bray \& McClaskey, 2015).

Als weitere sozialpsychologische Merkmale der Person lassen sich darüber hinaus die folgenden benennen:

- Akzeptanz des Entwicklungsprozesses (Bonsen, 2010; Feldhoff et al., 2008; Holtappels, 2014; Wissinger, 2014),

- (Selbst-)Reflexion der eigenen Überzeugungen, Werte und Haltungen hinsichtlich des Entwicklungsprozesses (Biermann, 2007; Gräsel \& Parchmann, 2004; Helmke, 2017),

- Aushandlung eines gemeinsamen Verständnisses, beispielsweise von zentralen Termini im Entwicklungsprozess (Bonsen, 2011; Ditton, 2000; Holtappels \& Feldhoff, 2010),

- Engagement und Verantwortungsübernahme (Biermann, 2007; Röhrich, 2008) und

- Thematisieren von Unsicherheiten, Irritationen und Ängsten sowie des persönlichen Leidensdrucks (Bonsen, 2010; Helmke, 2017).

\section{Zusammenführung der zwei Modelle}

Die beiden Modelle von Giaquinta (1973) respektive Giddens (1988) zeigen inhaltliche Überschneidungspunkte, denn in jeder Phase des Modells von Giaquinta (1973) kann eine Wechselwirkung von Strukturmoment und situativem Handeln stattfinden. Diese Überlegung lässt anhand eines synthetisierenden Schemas veranschaulichen (Abbildung 4.18).

Der grundlegende Gedanke des integrativen Modells ist im uSpL-Modell (siehe Abschnitt 4.2) enthalten. Es konnte allerdings wegen der begrenzten Darstellbarkeit in dieser Form nicht ins uSpL-Modell aufgenommen werden.

\subsection{Innerschulische Akteursgruppen in einer unterrichtszentrierten Schulentwicklung in Richtung personalisierten Lernens}

Die Entwicklung hin zu einer personalisierten Gestaltung von Lehr- und Lernprozessen wird primär von innerschulischen Akteursgruppen gestaltet. Dies sind auf 


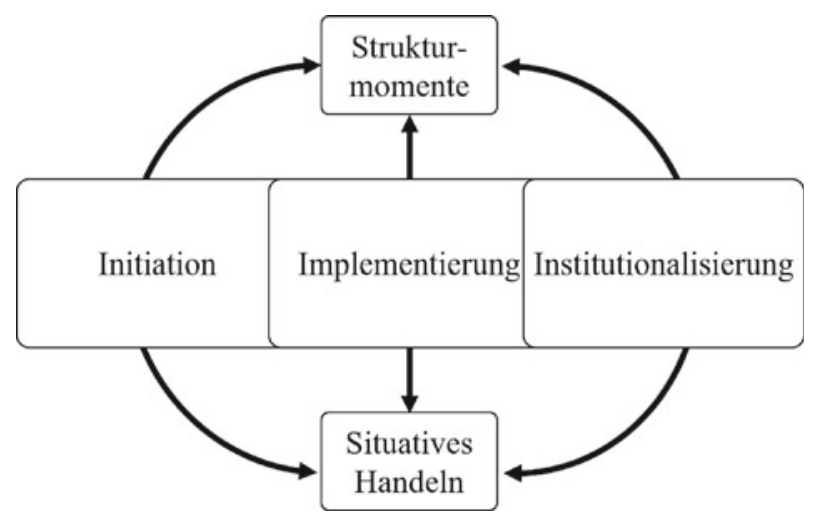

Abbildung 4.18 Kombination des Modells ,Wechselwirkung von Strukturmomenten und situativem Handeln“ (Giddens, 1988) und der Phasen „Initiation“, „Implementation“ und „Institutionalisierung“ (Giaquinta, 1973) (eigene Darstellung)

der einen Seite Schulleitende und ausgewählte Lehrpersonen, die den Entwicklungsprozess strategisch steuern. Auf der anderen Seite sind es die Lehrpersonengruppen, welche Entwicklungsvorhaben auf ihren Unterricht rekontextualisieren (Fend, 2008b). In solchen Prozessen kann es Personen geben, die in beiden Gruppen mitwirken. Dies ist zum Beispiel dann der Fall, wenn eine Lehrperson Mitglied einer Steuergruppe ist und gleichzeitig in einer Projektgruppe mitwirkt. Ausgerichtet ist das Handeln beider Gruppen jedoch auf denselben Entwicklungsgegenstand, das heißt auf eine stärkere Personalisierung - im Sinne der fünf Dimensionen personalisierten Lernens (siehe Abschnitt 3.2) - von Lehrund Lernprozessen, und bezieht sich beispielsweise auf die Erarbeitung von Stoffplänen für alters- und/oder leistungsdurchmischte Lerngruppen oder auf die Einführung von individuellen Coaching-Gesprächen (Abbildung 4.19).

Im Folgenden wird näher auf die Aufgaben- und Verantwortungsbereiche der Entwicklungsleitenden (Abschnitt 4.7.1), der umsetzenden Lehrpersonen (Abschnitt 4.7.2) sowie der Schülerinnen und Schüler (Abschnitt 4.7.3) eingegangen. 


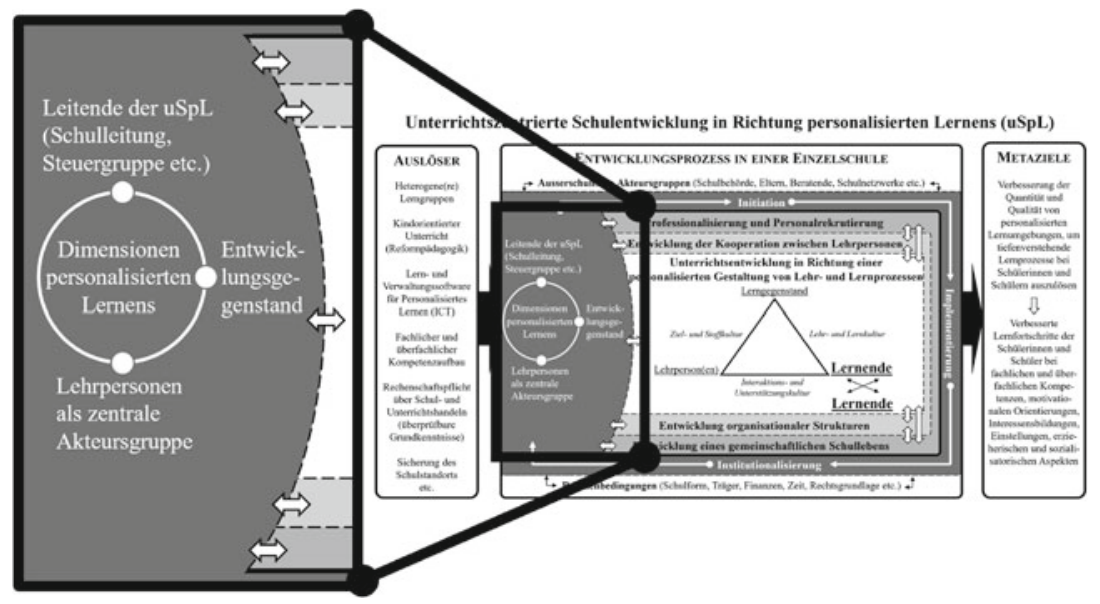

Abbildung 4.19 Die Wechselwirkungen zwischen Entwicklungsgegenstand, Leitenden der unterrichtszentrierten Schulentwicklung in Richtung personalisierten Lernens und Lehrpersonen im uSpL-Modell (eigene Darstellung)

\subsubsection{Personen für die Steuerung und Leitung von Entwicklungsprozessen}

Ein Entwicklungsprozess wird oftmals von Schulleitenden, häufig in Zusammenarbeit mit beauftragten Lehrpersonen in Steuergruppen oder erweiterten Schulleitungen, initiiert und geleitet. Konkret kann die Verteilung von Aufgabenund Verantwortungsbereichen sehr unterschiedlich aussehen.

\section{Funktion von Schulleitenden in einer unterrichtszentrierten Schulentwicklung} Mit der Einführung von Schulleitungen wurde in Schulen eine Führungsposition mit Management- und Verwaltungsaufgaben geschaffen. Ein Aufgaben- und Verantwortungsbereich besteht in der Initiation und der Steuerung von Weiterentwicklungen des Unterrichts und der Schule (Wissinger, 2016). Forschungsergebnisse zeigen, dass Schulleitende Schlüsselpersonen für Veränderungen sind. Als systemplayer (Fullan, 2016) obliegt ihnen die Aufgabe, in Kooperation mit Akteursgruppen der Systemebene (z. B. lokale Schulbehörde) strategische Leitlinien und Ressourcen für Entwicklungsprozesse auszuhandeln oder sich mit Schulleitenden anderer Schulen zu vernetzen, um Impulse und Inspiration für Weiterentwicklungen zu erhalten (Bonsen, 2010, 2011; Feldhoff et al., 2008; 
Fend, 2008b; Fullan, 2009; S. Huber, Wolfgramm \& Kilic, 2013; S. G. Huber, Sturm \& Köpfer, 2017; Köpfer \& Mejeh, 2017; Wissinger, 2014, 2016).

Im angloamerikanischen Sprachraum wird das Konzept einer Instructional Leadership als wirkungsvoll erachtet. Schulleitende, die sich an diesem Konzept orientieren, haben hohe Erwartungen an Lehr- und Lernprozesse, kommunizieren diese transparent und fordern sie ein. Steuerungsinstrumente sind beispielsweise standardisierter Leistungstests und die daran aufbauenden Interventionen (Granzer, Berger \& Looss, 2016; Hattie, 2009; Meyer, 2015; Scheerens, 2012). Allerdings weisen jüngste Studien darauf hin, dass Lehrpersonen durch das starke Instruieren weniger Verantwortung für das Lernen der Schülerinnen und Schüler übernehmen und dies zu Deprofessionalisierung führen kann (Metha, 2013).

Um dieser Tendenz entgegenzuwirken, werden Führungskonzepte erarbeitet, in denen die gemeinsame Verantwortung einerseits für Lernprozesse der Schülerinnen und Schüler und andererseits für Schul- und Unterrichtsentwicklung vermehrt betont wird. Als Beispiele hierfür lassen sich u. a. die Konzepte Leadership for Learning (MacBeath \& Dempster, 2009) und Learn Leader (Fullan, 2016) nennen. Geführt wird mit einem gemeinsamen Diskurs innerhalb der Schule über Vorstellungen und Auffassungen von Lehren und Lernen. Zudem wird das verantwortungsvolle Bearbeiten der Aufgabenbereiche jeder einzelnen Person hervorgehoben und gestärkt (Anderegg, 2015; Townsend, 2014). Aspekte solcher kooperativen, auf das Lernen der Schülerinnen und Schüler ausgerichteten Führungsstile weisen hohe Effektstärken auf die Lernleistungen auf, wie Robinson (2011) in ihrer Metaanalyse von zwölf Studien aufzuzeigen vermochte. Aus ihren Ergebnissen extrahierte sie schließlich fünf Dimensionen einer studentcentered leadership:

- Establishing goals and expectations $(d=.42)$ : Die Schulleitenden definieren, kommunizieren und evaluieren Lernziele und -standards in einer konsensuellen Zusammenarbeit mit den Lehrpersonen und weiteren Mitwirkenden.

- Resourcing strategically $(d=.31)$ : Unter einer strategischen Ressourcenbeschaffung sind die Ausrichtung der Ressourcenauswahl und -zuweisung auf die priorisierten Lernziele sowie durch Personalrekrutierung bereitgestelltes Fachwissen zu verstehen.

- Ensuring quality teaching $(d=.42)$ : Die Sicherstellung eines qualitativ hochwertigen Unterrichts umfasst die Planung, Koordination und Evaluation von Unterricht und Curriculum. Die Schulleitenden unterstützen und evaluieren die Lehrpersonen durch regelmäßige Hospitationen und mit formativem sowie summativem Feedback. 
- Leading teacher learning and development $(d=.84)$ : Schulleitende unterstützen und fördern Lehrpersonen in formellen und informellen Professionalisierungsprozessen.

- Ensuring an orderly and safe environment $(d=.27)$ : Die Schulleitenden sichern die Zeit für den Unterricht und das Lernen durch die Reduktion externen Drucks und den Aufbau und den Erhalt eines strukturierten und unterstützenden Umfelds.

In Anlehnung an angloamerikanische Führungsstile wird im deutschsprachigen Bildungsraum häufig das Konzept einer pädagogischen Schulführung vertreten (u. a. Bauer, 2008; S. G. Huber et al., 2017). Auch hier steht das Lernen der Schülerinnen und Schüler im Zentrum. Lehrpersonen und Schulleitende sind angehalten, stetig über ihr eigenes Handeln im Team bezüglich der Auswirkungen auf das Lernen der Schülerinnen und Schüler zu reflektieren. Unter einer pädagogischen Schulführung wird zudem die gemeinsame Verantwortung für die Schule subsumiert: Aufgaben- und Verantwortungsbereiche werden auf verschiedene Subteams im Kollegium verteilt. Diese Teams erhalten Handlungsspielräume, in denen Aufgaben selbstbestimmt bearbeitet werden können. Die Schulleitung hat diesbezüglich u. a. die Aufgabe, Kooperationsstrukturen zu schaffen, in denen Lehrpersonen ihr Fachwissen austauschen und dieses so aufeinander beziehen können, dass es dem Lernen der Schülerinnen und Schüler dient (Anderegg, 2016). Die Ausrichtung der Entwicklungstätigkeiten auf ein gemeinsames Verständnis von Schule und Unterricht ist in diesem Zusammenhang grundlegend.

Vor diesem allgemeinen Hintergrund ist im Kontext einer unterrichtszentrierten Schulentwicklung in Richtung einer personalisierten Gestaltung von Lehr- und Lernprozessen von Interesse, mit welchem Führungsstil Schulleitende einen positiven Einfluss auf das Lehr- und insbesondere auf das Lerngeschehen ausüben können. In einer Studie über sechs Schulen von Cervone und Cushman (2012) wurde u. a. das Schulleitungshandeln untersucht. In diesen Schulen übernehmen Schulleitende die Funktion eines teacheradvisor. Sie beraten und unterstützen Lehrpersonen dabei, den Wandel zu ihrem schulspezifischen Konzept personalisierten Lernens zu vollziehen und Herausforderungen und Probleme in der alltäglichen Unterrichtsarbeit zu lösen. Im Gegensatz zu diesem Befund konnte in einer Studie von Phillips und Cohen (2015) nachgewiesen werden, dass unterstützende Maßnahmen gerade bei jüngeren Lehrpersonen oftmals fehlen. Hier stellt sich die Frage, unter welchen intra und interpersonalen sowie strukturellen Bedingungen Unterstützung und Beratung vonseiten der Schulleitenden erfolgen. 
Hierzu liegen in der einschlägigen Literatur bislang keine empirischen Ergebnisse vor.

Leitung einer unterrichtszentrierten Schulentwicklung in Richtung personalisierten Lernens durch Gruppen von Schulleitenden und Lehrpersonen

Neben Schulleitenden können wie bereits festgehalten auch Gruppen von Schulleitenden und Lehrpersonen die Schulentwicklungsprozesse leiten und steuern. Dies kann beispielsweise in erweiterten Schulleitungen - das heißt Gremien, in denen Lehrpersonen mitwirken, die zusätzlich zu ihrem Unterrichtsauftrag Teilaufgaben der Schulleitung übernehmen - und in Steuergruppen geschehen. Die Steuergruppen werden im Folgenden näher beleuchtet.

Steuergruppen setzen sich meist aus Schulleitenden und Lehrpersonen jedes Fachbereichs respektive jeder Stufe zusammen. Sie sollen die einzelnen Subteams von Lehrpersonen in dem Gremium vertreten. Steuergruppen werden häufig in großen Schulen eingesetzt und leisten organisatorische Arbeiten, klären Rahmenbedingungen und gewährleisten zusammenhängende Entwicklungstätigkeiten aller innerschulischen Akteursgruppen. Bei der Steuerung und der Koordination der Schulentwicklung können Steuergruppen selbst Entwicklungsimpulse setzen oder Entwicklungsinitiativen der Lehrpersonen aufgreifen und diese mit den Zielen der Schule abgleichen. Darüber hinaus sammeln sie Informationen über die eigene Schule und beraten und unterstützen Schulleitende und Lehrpersonen (Holtappels, 2007; Holtappels, Pfeiffer, Röhrich \& Voss, 2008; S. G. Huber, 2011). Im Begleitforschungsprojekt ,Selbstständige Schule“ in NordrheinWestfalen (Deutschland) organisierten 87 Prozent der Steuergruppen schulinterne Fortbildungen zumeist zu Themen der Unterrichtsentwicklung, 52 Prozent berieten die Lehrpersonen. 74 Prozent der Steuergruppen halfen zudem bei der Planung von Projekten und 71 Prozent versuchten, Probleme zu lösen (Feldhoff et al., 2008).

\subsubsection{Entwicklung und Umsetzung eines Konzepts personalisierten Lernens durch Subteams von Lehrpersonen}

Während Schulleitende und Steuergruppen die innerschulischen Entwicklungsprozesse lenken und steuern, obliegen die konkrete Entwicklungsarbeit und die Umsetzung auf der Lehr- und Lernebene jeder einzelnen Lehrperson. Oftmals erfolgt die Durchführung von Entwicklungsprojekten in Teams (z. B. 
Jahrgangsteams, Stufenteams, Fachteams, pädagogische Teams etc.), die teilweise langjährig zusammenarbeiten. In solchen Strukturen ist jede einzelne Lehrperson gefordert, im Team gemeinsam entwickelte Konzepte einer stärker personalisierten Unterrichtsgestaltung auf der Stoff-, Lehr-Lern- oder der Lernunterstützungsebene in der alltäglichen Unterrichtsarbeit zu konkretisieren und umzusetzen, das heißt, den eigenen Unterricht konkret weiterzuentwickeln (Bray \& McClaskey, 2015; D. Hargreaves, 2006a; Prain et al., 2015a).

Bezogen auf den Entwicklungsprozess lassen sich verschiedene Haltungen von Lehrpersonen identifizieren: von enthusiastischen Befürworterinnen und Befürwortern bis zu eher kritischen Lehrpersonen, welche die Entwicklung ablehnen. In diesem Zusammenhang werden nachfolgend im ersten Teil des Unterkapitels zwei Modelle beschrieben, die die daraus resultierenden verschiedenen Rollen aufzeigen. Im zweiten Teil wird ein verändertes Rollenverständnis hin zu vermehrter ko-konstruktiver Kooperation zwischen Lehrpersonen und einem erweiterten und flexibler eingesetzten didaktischen Handlungsrepertoire beschrieben.

\section{Rollen von Lehrpersonen in einem Entwicklungsprozess}

Teil der pädagogischen Professionalität von Lehrpersonen (u. a. Rahm, 2010) ist es, sich an der Entwicklung von Schule und Unterricht zu beteiligen und diese mitzugestalten. So ist etwa im Kanton Zürich seit 2015 im neuen Berufsauftrag für Lehrpersonen festgelegt, dass Lehrpersonen, die 100 Prozent angestellt sind, im Jahr 60 Arbeitsstunden für erweiterte Tätigkeiten einsetzen können. Diese umfassen die Zusammenarbeit im Kollegium, mit den Schulbehörden und mit weiteren Amtsstellen sowie die Teilnahme an Sitzungen und die Übernahme von Aufgaben für die Schule. Ebenso aufgeführt werden die pädagogische Mitgestaltung und die Qualitätssicherung und -entwicklung der Schule (Volksschulamt Kanton Zürich, 2015).

Forschungsergebnisse zeigen, dass in der Regel nicht alle Lehrpersonen in gleichem Masse ihre Schule und den Unterricht weiterentwickeln wollen. So sehen einige ihre teils traditionellen subjektiven Theorien und Auffassungen von Unterrichten bedroht. Auch Überlastungen oder Zweifel an der Sinnhaftigkeit von Entwicklungsprojekten können Gründe sein, warum Lehrpersonen sich weigern, Veränderungen mitzutragen (Altrichter \& Feyerer, 2017). Ist es im Entwicklungsprozess nicht oder nur teilweise gelungen, kognitive Wissensbestände und subjektive Theorien aller beteiligten Lehrpersonen zu verändern, kann dieser scheitern (Nowinska, 2010; Schlee, 2014).

Neben Lehrpersonen, die sich gegen Entwicklungsprojekte stellen, gibt es Lehrpersonen, welche diese besonders vorantreiben. Diese Spannweite fasste Rogers (1983) in einer theoretischen Verteilung zusammen (Abbildung 4.20). 


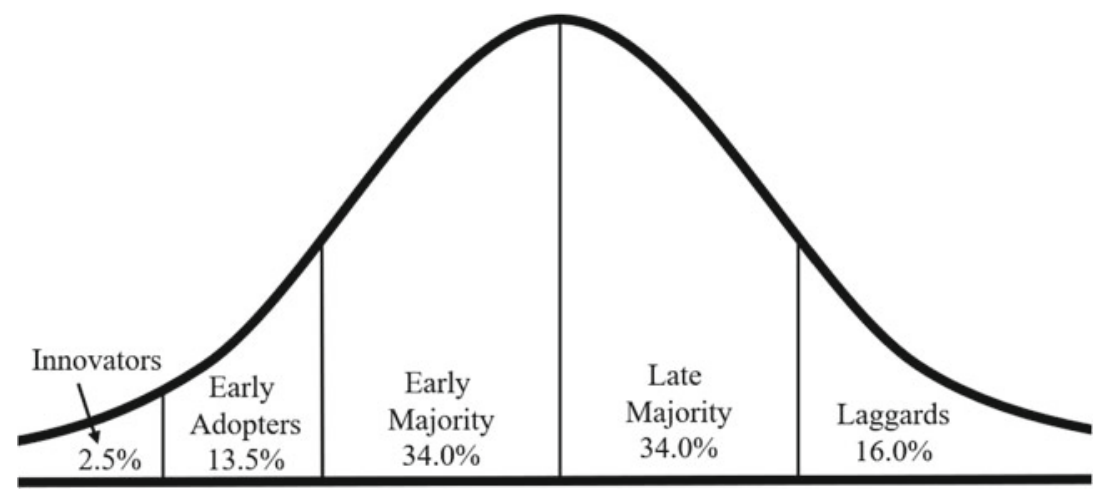

Abbildung 4.20 Theoretische Verteilung von Lehrpersonen und ihrer Akzeptanz gegenüber dem Entwicklungsprozess (Rogers, 1983)

Die Einteilung von Rogers (1983) umfasst insgesamt fünf Gruppen. Die innovators sind die ersten Personen im Team, welche eine Innovation annehmen und akzeptieren. Danach folgen die early adopters. Sie sind motiviert und integrieren die Innovation relativ schnell in ihren Berufsalltag. Bei den zwei größten Gruppen, nämlich der early und der late majority, bedarf es Überzeugungsarbeit und Zeit, damit sie die Veränderungen mittragen, wobei Personen der late majority eher zurückhaltender und skeptischer sind als jene der early majority. Die letzte Gruppe sind die laggards, welche Innovationen eher widerwillig akzeptieren und übernehmen oder in Ablehnung verharren (Holtappels, 2014; Rogers, 1983; Schrifter, 2016). Eine in ähnlicher Form vorgenommene Unterscheidung von Rehnmann und Härnwall (1991, zitiert nach Schratz \& Steiner-Löffler, 1999) differenziert zwischen „Missionierenden“, „Gläubigen“, „Lippenbekennenden“, ,abwartenden Gleichgültigen“, „Untergrundkämpferinnen und Untergrundkämpfern“, ,,aufrechten Gegnerinnen und Gegnern“ sowie „Emigrantinnen und Emigranten“. Auch hier wurden die Personengruppen einer theoretischen Normalverteilung zugeordnet, bei der die abwartenden Gleichgültigen die mittlere und zugleich größte Gruppe bilden. Speziell hinzuweisen gilt es bei dieser Darstellung auf die Gruppe der Emigrantinnen und Emigranten: Diese umfasst Personen, die mit der Entwicklung nicht einverstanden sind und diese nicht mittragen möchten, weshalb sie die Schule verlassen. Insbesondere in sehr entwicklungsfreudigen Schulen gibt es Lehrpersonen, die aufgrund der Entwicklungstätigkeiten ihre Stelle aufgeben oder neu dazukommen. 
Verändertes Rollenverständnis von Lehrpersonen

Wenn Konzepte personalisierten Lernens umgesetzt werden, finden vermehrt Phasen ko-konstruktiver Kooperation zwischen Lehrpersonen statt und die Schülerinnen und Schüler werden vermehrt in ihren Lernprozessen gecoacht und beraten. Entschließen sich Schulleitende und Lehrpersonen dazu, zu diesem Zweck in Stufen- und/oder Jahrgangsteams zusammenzuarbeiten und die Lerngruppenzusammensetzung in den Stufen und/oder Jahrgängen zu flexibilisieren, dann müssen Lehrpersonen fortan stärker kooperieren, und zwar nicht nur bezogen auf den Entwicklungsprozess, sondern auch in ihrer alltäglichen Unterrichtsarbeit. Bereits im Kapitel über die Entwicklung der Kooperation zwischen Lehrpersonen (siehe Abschnitt 4.5.3) wurde beschrieben, dass diese Veränderungen eine häufigere ko-konstruktive Kooperation mit sich bringen. Dies stellt besondere Anforderungen an die Lehrpersonen, denn einerseits sollten sie über professionelle Kommunikationskompetenzen verfügen, um den kooperativen Raum konstruktiv zu bearbeiten, und andererseits sollten sie gegenüber einer intensiven Teamarbeit eher positiv eingestellt sein.

Bezüglich der Lernunterstützung von Schülerinnen und Schülern werden die Rollen vielfältiger. Lehrpersonen sind nicht mehr nur reine Stoffvermittelnde, sondern in einem personalisierten Lernumfeld (student-driven) vermehrt „facilitator and partner in learning“ (Bray \& McClaskey, 2015, S. 73). Als Lernberatende und Lerncoaches unterstützen Lehrpersonen das Lernen von Schülerinnen und Schülern adaptiv mit kognitiv anregendem, stärker sokratischem (Nach-)Fragen sowie qualifiziertem fachlichem und überfachlichem Feedback und Feedforward (Cervone \& Cushman, 2012; Perkhofer-Czapek \& Potzmann, 2016; Stebler et al., 2017; Zmuda et al., 2015). Das veränderte Rollenverständnis professionellen Handelns von Lehrpersonen geht mit der Herausforderung einher, dass hierzu subjektive Theorien verändert und teils neue Fertigkeiten und Handlungskompetenzen erworben oder vertieft werden müssen. Dies sind Prozesse, die Zeit brauchen und die nicht auf uniformen Wegen für alle gleich verlaufen (Phillips \& Cohen, 2015).

\subsubsection{Schülerinnen und Schüler}

Die Rolle der Schülerinnen und Schüler in einer unterrichtszentrierten Schulentwicklung in Richtung personalisierten Lernens lässt sich unter dem Aspekt der Partizipation in der didaktischen Unterrichtsgestaltung sowie unter dem Aspekt der Partizipation in Prozessen der Schul- und Unterrichtsentwicklung erläutern. 
Unabhängig davon, welche Form der Partizipation im Unterricht oder im Entwicklungsprozess vorgesehen wird, bedarf es für deren Erfolg vonseiten der Lehrpersonen einer stetigen Reflexion des eigenen pädagogischen und didaktischen Handelns. Nur wenn eigene Handlungsweisen stetig hinterfragt werden und darüber nachgedacht wird, inwiefern diese die persönlichen Lernprozesse der Schülerinnen und Schüler fördern, kann Partizipation gelingen (Moldenhauer, 2015). Gründe für ein Nichtgelingen sind beispielsweise Zweifel der Lehrpersonen an den Kompetenzen der Schülerinnen und Schüler oder die Annahme, dass die Lernenden den Raum der Mitgestaltung nicht erwartungsgemäß bearbeiten. Bisweilen wird auch befürchtet, dass die Autorität der Lehrpersonen angezweifelt und untergraben werden könnte oder zu wenig Zeit für Partizipation vorhanden ist (Lundy, 2007).

\section{Partizipation in der didaktischen Gestaltung von Unterricht}

Einem konstruktivistischen Lernverständnis zufolge (siehe Abschnitt 4.5.1.1) sind Schülerinnen und Schüler keine passiven Unterrichtsteilnehmenden, die Lerninhalte einfach nur rezipieren, und auch keine „unbeschriebenen Blätter“ („tabula rasa“), die von Lehrpersonen „beschrieben“ werden. Schülerinnen und Schüler lernen vielmehr aktiv und auf der Basis von Vorwissen. Aktives Lernen wird in diesem Kontext als verstehens- und problemorientierte Auseinandersetzung mit Lerninhalten verstanden, die tief durchdrungen werden, sodass dahinterliegende Bedeutungsstrukturen verstanden und mit dem eigenen Vorwissen verknüpft werden können. In dem Masse, wie die Schülerinnen und Schüler ihr Lernen selbst steuern, werden sie zu Koproduzentinnen und Koproduzenten ihrer eigenen Lernergebnisse. Damit sie ihre Lernprozesse aktiv gestalten und regulieren können, benötigen sie Lernstrategien und personale Kompetenzen. Sie müssen Lerntechniken erlernen, ausprobieren und in verschiedenen Kontexten anwenden, um sich fachliche und überfachliche Kompetenzen aneignen zu können. Lehrpersonen können die individuellen Lernprozesse interaktiv unterstützen, zum Beispiel durch die Modellierung von Zieltätigkeiten, Scaffolding, Feedback und formative Unterstützung (siehe Abschnitt 4.5.1). Ebenso ist es ihre Aufgabe, eine Lernumgebung zu schaffen, in denen die Schülerinnen und Schüler die Möglichkeiten haben, aktiv am Lehr- und Lerngeschehen teilzunehmen (Oelkers \& Reusser, 2008). Möglichkeiten der Mitgestaltung in Lernumgebungen ergeben sich nach Bohl und Kucharz (2010), die sich wiederum an den Arbeiten von Peschel (2005) und Ramseger (1985) orientieren, in fünf Stufen:

- „1. Stufe: organisatorische Öffnung (Inwieweit können die Schüler/innen ihre Rahmenbedingungen selbst bestimmen?) 
- 2. Stufe: methodische Öffnung (Inwieweit kann die Schülerin/der Schüler ihrem/seinem eigenen Lernweg folgen?)

- 3. Stufe: methodische und inhaltliche Öffnung (Inwieweit kann die Schülerin/der Schüler darüber hinaus über ihre/seine Lerninhalte bestimmen?)

- 4. Stufe: politisch-partizipative Öffnung (Inwieweit können die Schüler/innen in der Klasse Unterrichtsablauf und -regeln mitbestimmen?)

- 5. Stufe: Öffnung der Schule (Inwieweit öffnet sich die Schule nach außen zur Umwelt und lässt die Außenwelt herein?)“ (Bohl \& Kucharz, 2010, S. 85).

Dieses Stufenmodell dient der Charakterisierung von offenem Unterricht in Abgrenzung zu einer Öffnung des Unterrichts. Für die vorliegende Arbeit relevant sind in diesem Zusammenhang die Möglichkeiten der Mitgestaltung im Unterricht. Allerdings sind die Stufen nicht in allen Belangen trennscharf: So wird in den Ausführungen zum Beispiel nicht definiert, was unter den „Rahmenbedingungen“ (organisatorische Dimension) zu verstehen ist. Damit könnten beispielsweise auch Verhaltensregeln im Lernzimmer gemeint sein (partizipativpolitische Dimension). Auch die Unterscheidung zwischen „eigenem Lernweg folgen“ und „Lerninhalte selbst bestimmen“ ist insofern nicht gänzlich nachvollziehbar, als die Lerninhalte Teil des Lernwegs sein können. Angesichts dieser konzeptionellen Schwierigkeiten lohnt sich der Blick auf eine weitere, an das Stufenmodell anschließende Differenzierung von Bohl und Kucharz (2010). Sie definieren vier Varianten von inhaltlich-unterrichtsbezogenen Mitgestaltung der Schülerinnen und Schüler. Die erste Variante wird stark von der Lehrperson festgelegt, während die letzte stark von den Schülerinnen und Schülern bestimmt wird. Variante zwei und drei sind Mischformen:

- „Der Lehrer gibt genau vor, [welche Schülerin und] welcher Schüler welches Thema/welche Aufgabe bearbeitet.

- Die Schülerinnen und Schüler wählen ein Thema/eine Aufgabe aus mehreren und anspruchsvollen Angeboten aus.

- Die Schülerinnen und Schüler bestimmen selbst ein (Teil-)Thema aus einem vorgegebenen Rahmenthema.

- Die Schülerinnen und Schüler entscheiden frei, welches Thema sie bearbeiten“ (Bohl \& Kucharz, 2010, S. 20).

Im Kontext personalisierten Lernens stellt sich hier die Frage, welche Variante in welchen Kontexten für den Lernerfolg der Schülerinnen und Schüler am wirkungsvollsten ist. Im Kontext einer reformpädagogischen Argumentation lässt sich diesbezüglich die Hypothese formulieren, dass selbst gewählte Inhalte für 
Schülerinnen und Schüler sehr bedeutungsvoll sind und zu bedeutsamen Lernprozessen führen können, auf deren Grundlage die Schülerinnen und Schüler motiviert und tiefgründig die Lerninhalte durcharbeiten. Demgegenüber ist die Lehrperson - besonders in der Volksschule - jedoch dazu verpflichtet, die Inhalte des Lehrplans mit den Schülerinnen und Schülern zu bearbeiten, weshalb gewisse Lerninhalte vorgeben muss. Es gilt daher sicherzustellen, dass auch die gesetzten Lerninhalte bei den Schülerinnen und Schülern auf Interesse stoßen und zu bedeutsamen Lernprozessen führen.

\section{Partizipation in Prozessen der Schul- und Unterrichtsentwicklung}

Neben der Partizipation im Unterrichtsgeschehen besteht auch die Möglichkeit, dass Schülerinnen und Schüler in Schulentwicklungsprozessen mitwirken. Dies ist bisher wenig erforscht (Feichter, 2017; Fullan, 2016). In Konzepten personalisierten Lernens lassen sich jedoch Elemente identifizieren, bei denen die Schülerinnen und Schüler an den Entwicklungsprozessen teilhaben können. Die Idee von Voice and Choice (Miliband, 2006) bzw. der erweiterten Handlungs- und Autonomiespielräume des eigenen Lernprozesses (Bray \& McClaskey, 2015; Stebler et al., 2018) eröffnet Potenziale, die es den Schülerinnen und Schülern ermöglichen, direkt oder indirekt Entwicklungsimpulse setzen. Einerseits finden sich die Schülerinnen und Schüler in Lernumgebungen, welche die Lehrpersonen verstärkt an die individuellen Lernprozesse anpassen. So kann es beispielsweise sein, dass in einem Jahrgang viele Schülerinnen und Schüler Schwierigkeiten damit haben, ihre Lernaufgaben für die nächsten Unterrichtsstunden aufzuteilen, einen Arbeitsplan zu erstellen und diesen einzuhalten. Wenn die Lehrpersonen es aus personellen Gründen nicht schaffen, den Schülerinnen und Schülern im Unterricht die dazu nötigen Kompetenzen zu vermitteln, bräuchte es weitere Unterstützungsangebote, die von Lehrpersonenteams entwickelt werden. Ein solches Unterstützungsangebot würde sich erübrigen, wenn nachfolgende Jahrgänge weniger Schülerinnen und Schüler umfassen würden. Andererseits können die Schülerinnen und Schüler durch Ideen und Anregungen, die sich auf die Nutzung der Lernfreiräume beziehen, bei den Lehrpersonen indirekt Entwicklungsimpulse setzen (u. a. Schratz, 2017; Schratz, Schwarz \& Westfall-Greiter, 2011). Eine weitere Möglichkeit besteht darin, dass die Schülerinnen und Schüler von den Lehrpersonen mündlich oder mit schriftlichen Befragungen um ihre Ansichten und Meinungen zum Lernen im jeweiligen Konzept personalisierten Lernens gebeten werden (Bonsen, 2010, 2011). 


\subsection{Außerschulische Akteursgruppen in einer unterrichtszentrierten Schulentwicklung in Richtung personalisierten Lernens}

Unterrichtszentrierte Schulentwicklung in Richtung personalisierten Lernens wird nicht nur von innerschulischen Akteursgruppen gestaltet, sondern ist immer eingebettet in einen lokalen Bildungsraum und wird auch von Akteursgruppen der Systemebene, zum Beispiel der lokalen Schulbehörde, mitgestaltet. Akteursgruppen außerhalb der Einzelschule arbeiten mit Schulleitenden und Lehrpersonen zusammen und können Entwicklungsprozesse ebenfalls beeinflussen (Abbildung 4.21). Diesbezüglich lassen sich vier bedeutsame Akteursgruppen unterscheiden (u. a. Altrichter \& Helm, 2011; OECD, 2006; Saalfrank, 2016): erstens Schulbehörde (Abschnitt 4.8.1), zweitens Eltern (Abschnitt 4.8.2), drittens Beratende (Abschnitt 4.8.3) und viertens Mitglieder schulischer Netzwerke (Abschnitt 4.8.4). Deren Möglichkeiten der Einflussnahme werden nachfolgend beschrieben.

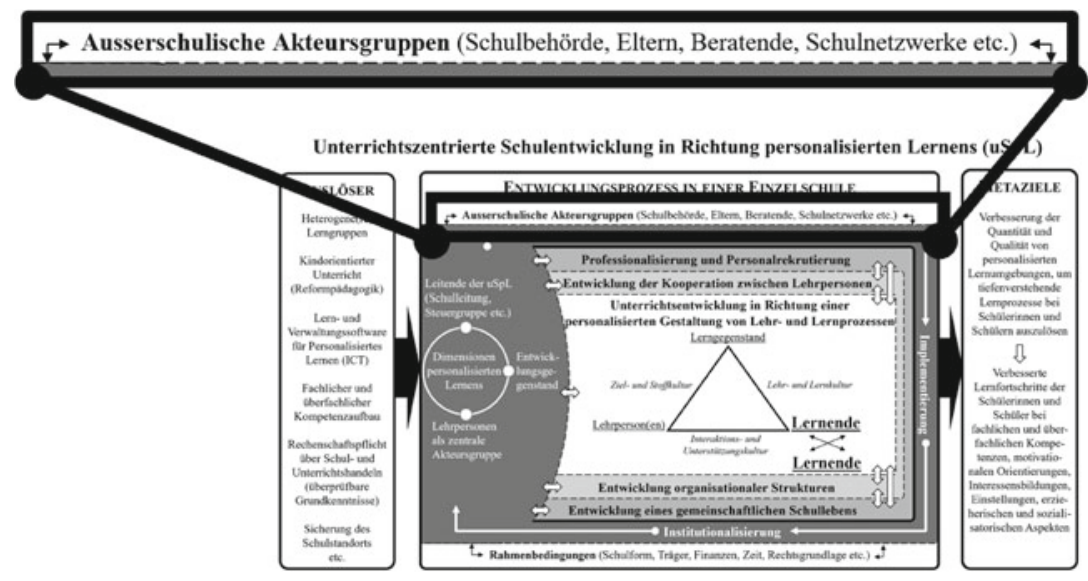

Abbildung 4.21 Außerschulische Akteursgruppen im uSpL-Modell (eigene Darstellung) 


\subsubsection{Schulbehörde}

Die Schulbehörden haben u. a. die Aufgabe, den strukturellen, den gesetzlichen und den finanziellen Rahmen für den Entwicklungsprozess festzulegen, diesen zu kontrollieren und den einzelnen Schulen Handlungsspielräume zu eröffnen oder diese gegebenenfalls einzugrenzen (u. a. Bonsen, Bos \& Rolff, 2008; Rolff, 2013). Ein wichtiger Aspekt hierbei ist die pädagogische Autonomie, die es Schulleitenden und Lehrpersonen erlaubt, über pädagogische Themen zu entscheiden und deren Bearbeitung verantwortungsvoll wahrzunehmen (Weigand, 2004). Des Weiteren können Schulbehörden Entwicklungsprozesse in Einzelschulen einerseits gezielt initiieren, indem sie die Schulen in ihrer Bildungsregion zur Umsetzung eines Entwicklungsprojekts verpflichten. Für wirkungsvolle und nachhaltige Schul- und Unterrichtsentwicklung ist jedoch auch in diesem Fall eine unterstützende Zusammenarbeit zwischen den Verantwortlichen der Schulbehörde und der Einzelschule notwendig (Fullan, 2009). Andererseits können durch Veränderungen der Rahmenbedingungen auch von der Schulbehörde nicht direkt intendierte Entwicklungsimpulse gesetzt werden. Droht etwa die Schulschließung, weil zu wenig Kinder im Einzugsgebiet wohnen und das Unterrichtsmodell finanziell nicht mehr tragbar ist, nehmen das einige Schulleitende und Lehrpersonen zum Anlass, ihr Modell umzustrukturieren und beispielsweise altersdurchmischtes Lernen einzuführen, um auf diese Weise den Schulstandort zu sichern (Sommer-Sutter, 2012).

\subsubsection{Eltern}

Neben den Lehrpersonen sind auch die Eltern wichtig für den Lernerfolg ihrer Kinder. Je mehr die Eltern über schulische Bildung und Erziehung wissen und sich daran beteiligen, desto grösser ist ihre Wirkung auf die Entwicklung und die schulischen Erfolge (Fullan, 2016). Sacher (2014) gelangte in einem Forschungsreview zum Schluss, dass Schulen von der Zusammenarbeit mit Eltern profitieren, wenn diese über die Kontaktpflege, den Informationsaustausch und die Schaffung einer guten Stimmung und Atmosphäre hinausgehe. Der Autor plädiert daher für eine Erziehungs- und Bildungspartnerschaft, bei der eine von Schule und Eltern gemeinsam getragene, kooperative Unterstützung der schulischen und persönlichen Entwicklung des Kindes bzw. der oder des Jugendlichen im Zentrum steht. Eine solche Partnerschaft sollte einerseits das aktive Mitwirken der Eltern in Schule und Unterricht umfassen, indem diese Aufgaben übernehmen können und Entscheidungsbefugnisse erhalten. Andererseits lässt sich durch die 
intensive Kooperation ein entwicklungsförderliches Erziehungsverhalten anregen und es können produktive häusliche Lernumgebungen geschaffen werden.

In eine ähnliche Richtung argumentieren Wild und Lütje-Klose (2017). Aus dem US-Sprachraum adaptierten sie die Standards for Family-School Partnership für inklusive Schulen und formulierten Orientierungshilfen für eine solche Erziehungs- und Bildungspartnerschaft: Lehrpersonen und Schulleitende sollten eine Willkommens- und Begegnungskultur schaffen und etablieren, um allen Eltern Zugang zur Schule zu ermöglichen, in der ein regelmäßiger, produktiver, effizienter Informationsaustausch stattfindet. In einer solchen Kultur stimmen Eltern, Schülerinnen und Schüler und Lehrpersonen im Inklusionskontext gemeinsam Lernziele und -inhalte ab und klären Verantwortlichkeiten für die Ausarbeitung und die Umsetzung individueller Förderpläne. Gesprächsführungsund Beratungskompetenzen von Lehrpersonen werden gestärkt, um Ressourcen in Elternhäusern für die Unterstützung der Kinder zu erkennen und auszuweiten. Formale Strukturen werden eingerichtet, in deren Rahmen möglichst alle Eltern ihre Rechte und Pflichten der Mitbestimmung in Schule und Unterricht wahrnehmen können. Dies beinhaltet u. a., dass sich Eltern in Fragen der Gestaltung des Schullebens und des Unterrichts einbringen können.

Darüber hinaus gilt es darauf hinzuweisen, dass Eltern über Potenziale verfügen, Schulentwicklungsprozesse zu bereichern. So können sie beispielsweise ihre Außenperspektive zu Entwicklungstätigkeiten einbringen und dadurch Impulse setzen (Kurz \& Weiß, 2016). Allerdings werden Eltern in Konzepten und Modellen von Schul- und Unterrichtsentwicklung sehr häufig nur am Rand thematisiert. Ein Grund dafür sind gesetzliche Vorgaben: So wird den Eltern zum Beispiel im Kanton Zürich die Mitwirkung in der didaktischen Gestaltung des Unterrichts gesetzlich nicht zugestanden (Volksschulgesetz Kanton Zürich, 2005, § 55). Des Weiteren könnte vermutet werden, dass Eltern weniger als Mitwirkende, sondern eher als Kundinnen und Kunden oder Regulative verstanden werden, die zum Beispiel über Evaluationen kritische Rückmeldungen zu Entwicklungsprozessen geben (Meister, 2010). Forschungsergebnisse zeigen, dass Elternbeteiligung und -mitwirkung in Entwicklungsprozessen eher selten sind (Forschungsgruppe WissGem, 2016; Meister, 2010).

Im Forschungsprojekt perLen ergaben Analysen von Fragebogendaten der Lehrpersonen, dass sich die Qualität der Zusammenarbeit mit den Eltern durch die Schul- und Unterrichtsentwicklung in Richtung personalisierten Lernens kaum verändert hatte. In sehr stark personalisierten Schulen wurde die Zusammenarbeit mit Eltern jedoch als produktiver eingeschätzt als in moderat personalisierten Schulen (Gruppendefinition siehe Abschnitt 4.5.1.4) (Stebler et al., 2017). In 
einer Fallanalyse von zwölf Schulen desselben Projekts wurde die von den Lehrpersonen und den Schulleitenden in Interviews berichtete Zusammenarbeit mit Eltern untersucht (Vasarik Staub, Stebler \& Reusser, 2018). Eine typenbildende Inhaltsanalyse ergab drei Typen: Zu Typ 1 gehören Schulen mit informaler Kommunikation und Kooperation. Dies sind kleinere Schulen (ca. 100 Schülerinnen und Schüler), in denen verstärkt auf informeller Ebene mit Eltern zusammengearbeitet wird. Eltern und Lehrpersonen besprechen Themen wie Hausaufgaben und Lernleistungen der Kinder oder deren Entwicklungsmöglichkeiten. Typ 2 wurden größere Schulen zugeordnet, die sich durch reaktive Formen der Kommunikation und Kooperation charakterisieren lassen. Wenn Eltern Gesprächsbedarf haben und sich diesbezüglich bei der zuständigen Lehrperson melden, findet eine Zusammenarbeit statt. Ansonsten erfolgt die Zusammenarbeit vor allem an offiziellen Schulveranstaltungen wie etwa Schulfesten oder Elterngesprächen. Weitere Formen von Zusammenarbeit hängen bei diesem Typ von den individuellen Einstellungen einzelner Lehrpersonen ab. Typ 3 schließlich umfasst Schulen mit strategischer Kommunikation und Kooperation. Es bestehen verbindliche Kooperationsstrukturen, in denen hauptsächlich pädagogische Fragen diskutiert werden. Strategisch ist dieses Konzept insofern, als verschiedene institutionelle und individuelle Kommunikationswege implementiert und bekannt sind, die sowohl von den Lehrpersonen als auch von den Eltern genutzt werden können. Dazu gehört zum Beispiel das Miteinbeziehen von Eltern in die Entscheidungsfindung bei Entwicklungsprozessen auf Schulebene.

\subsubsection{Schulexterne Beratende}

Eine weitere außerschulische Akteursgruppe bilden Beratende. Deren Aufgabenbereiche in Schulentwicklungsprozessen sind vielfältig. Sie erstrecken sich von der Begleitung und der Beratung einer ganzen Schule über mehrere Jahre hinweg, Beratungen einzelner Lehrpersonen zu Unterrichtsthemen über die Moderation und die Beratung von Lehrpersonengruppen bis hin zu Evaluationstätigkeiten, bei denen Daten erhoben, ausgewertet und der Schule zurückgemeldet werden (Adenstedt, 2016; E. Arnold \& Reese, 2010; Buhren \& Rolff, 2012; Goecke, 2018; Knauf, Goecke \& Rauh, 2010). Dementsprechend vielfältig sind auch die Rollen der Beratenden: Es handelt sich um Fachexpertinnen und Fachexperten, Organisationsberaterinnen und Organisationsberater, Expertinnen und Experten zu Schulentwicklungsprozessen, Coachs zum Reflektieren und Problemlösen, Supervisorinnen und Supervisoren etc. (Buhren \& Rolff, 2012; Dedering, 2017; Knauf et al., 2010). In einem Forschungsreview von neun Studien zählte Tajik (2008) 
über 20 Rollen von Beratenden in Schulentwicklungsprozessen auf. Der Autor fasste diese zu drei Gruppen zusammen:

- Unterstützerinnen und Unterstützer (facilitators) von Lehrpersonen: Die Beraterinnen und Berater geben Anregungen zur pädagogischen und didaktischen Unterrichtsgestaltung und befähigen Lehrpersonen dazu, Entwicklungsprozesse selbst zu gestalten.

- Kritische Freundinnen und Freunde (critical friends): Die Beratenden geben positive und kritische Rückmeldungen zu innovativen Entwicklungsideen und -plänen.

- Technische Expertinnen und Experten (technical experts): Diese Personen geben aufgrund ihrer Expertise Hinweise, Richtlinien oder Vorgaben für möglichst erfolgreiche Entwicklungsprozesse.

Arnold und Reese (2010) zählen ähnliche Rollen auf, jedoch mit einer Ausnahme: Beratende können auch eine Legitimationsfunktion ausüben. In diesem Zusammenhang werden sie als Fachexpertinnen und Fachexperten eines bestimmten Themengebiets eingeladen, wodurch sich Entwicklungsprozesse unter Verweis auf ihre Fachexpertise im Kollegium legitimieren lassen, um Befürworterinnen und Befürworter der Entwicklungsprozesse zu gewinnen. Quantitative Befragungen zur Beratungshäufigkeit ergaben, dass 50 Prozent (Adenstedt, 2016) bzw. 70 Prozent (Knauf et al., 2010) der befragten Schulleitenden mindestens eine Beratung in Anspruch genommen hatten. Besonders wichtig waren Beratungsthemen zur Unterrichtsentwicklung wie Inklusion, Individualisierung, Kompetenzorientierung oder Unterrichtsqualität (Adenstedt, 2016). Der Einsatz von externen Beratenden wird von Schulleitenden positiv bewertet und geht nach deren Einschätzung mit einem Kompetenz- und Akzeptanzzuwachs der Lehrpersonen in Entwicklungsprozessen einher (Dedering, 2013; Dedering, Tillmann, Goecke \& Rauh, 2013). Weitere Studien, insbesondere aus dem Schweizer Bildungsraum oder bezogen auf personalisiertes Lernen, zu Aufgabenbereichen, Funktionen und Wirkungen von Beratenden in Entwicklungsprozessen liegen in der einschlägigen Literatur bislang jedoch noch nicht vor. Spezifisch für den Kontext der Schweiz lassen sich demgegenüber einige Anbieterinnen und Anbieter für Schulberatungen finden. So gibt es Weiterbildungsangebote in und außerhalb von Schulen mit staatlich angestellten oder privaten Coachs sowie Fachexpertinnen und Fachexperten zu Themen der Schul- und Unterrichtsentwicklung. 


\subsubsection{Mitglieder schulischer Netzwerke}

In Schulnetzwerken treten Schulleitende und Lehrpersonen mit anderen Schulen über die eigenen regionalen Gebiete hinweg in Kontakt und tauschen sich über Ziele, Methoden oder Problemlösungen in Bezug auf Entwicklungsprozesse aus (Campbell et al., 2007; Cervone \& Cushman, 2012; Holtappels, 2013). Dies wird auch als „horizontale Schulentwicklung“ bezeichnet (Brühlmann \& Rolff, 2015). Solche Netzwerke können unterschiedliche Trägerschaften und Anlässe sowie unterschiedliche Zielsetzungen haben, beispielsweise die Ermöglichung eines themenbezogenen Erfahrungsaustauschs über die Professionalisierung der Lehrpersonen, die Erprobung von Konzepten, die Verbesserung des Unterrichts oder die Förderung der Schulkooperation (Berkemeyer, Lehmpfuhl \& Pfeiffer, 2010c). Berkemeyer, Manitius und Müthing (2008) unterscheiden diesbezüglich drei Netzwerktypen:

- soziale Netzwerke, in denen die Schulen untereinander soziale Beziehungen aufbauen, um etwa gemeinsame Aktivitäten durchzuführen (Ausflüge etc.),

- strategische Netzwerke, in denen die Schulen Allianzen mit anderen Schulen bilden, um spezielle Interessen gemeinsam zu verfolgen, und

- Innovationsnetzwerke, in denen eine Wissensgemeinschaft gebildet wird, um aufbauend auf dem Wissensaustausch Veränderungen zu initiieren.

Gerade der dritte Netzwerktyp weist hohe Potenziale im Hinblick auf die Anregung von Schul- und Unterrichtsentwicklung auf. Empirische Ergebnisse zeigen, dass durch die Netzwerkarbeit Reflexions- und Lernprozesse bei den beteiligten Lehrpersonen angeregt werden, die wiederum Impulse für die Schulund Unterrichtsentwicklung geben (Berkemeyer, Järvinen \& Ophuysen, 2010b; Järvinen, Manitius, Müthing \& Berkemeyer, 2015; Jungermann, Pfänder \& Berkemeyer, 2018). Wichtiges Qualitätsmerkmal ist in diesem Zusammenhang die Kooperationsqualität: Von Bedeutung ist professionelles Handeln mit Lern- und Arbeitsstrategien, die zu einem intensiven Austausch und einer tiefgehenden Bearbeitung von Schulentwicklungsprozessen anregen (Maag Merki, 2009). Damit die Impulse aus der Netzwerkarbeit in der eigenen Schule fruchten, braucht es einen Wissenstransfer. Dies bedeutet, dass sich an der Netzwerkarbeit beteiligte Lehrpersonen mit nicht beteiligten Lehrpersonen hinsichtlich der neuen Erkenntnisse austauschen und deren Entwicklungsmöglichkeiten abwägen. Studien zeigen, dass dieser Transfer häufig in „einfacheren“ oder „,niederschwelligen“ Kooperationsformen geschieht (z. B. unterrichtsbezogener Austausch von pädagogischen Ideen 
oder Austausch von im Netzwerk erarbeiteten Unterrichtsreihen und Problemlösungen). Anspruchsvollere Kooperationsformen wie beispielsweise diejenige der Unterrichtshospitation wurden lassen sich jedoch selten feststellen (Jungermann et al., 2018; Killus \& Gottmann, 2012). In Anbetracht des Professionalisierungspotenzials ko-konstruktiver Kooperation (siehe Abschnitt 4.5.3) stellt sich hier die Frage nach der Nachhaltigkeit der Zusammenarbeit in Netzwerken. Hierzu liegen kaum empirische Befunde vor.

In der Schweiz gibt es einige Schulnetzwerke, die sich mit der Frage auseinandersetzen, wie Unterricht zukünftig zu gestalten sei, damit zunehmend heterogene Lerngruppen bestmöglich unterrichtet werden können. Beispiele hierfür sind das Netzwerk im Kanton Luzern (Dienststelle Volksschulbildung des Kantons Luzern, 2012) und das Netzwerk ,heterogen lernen“ (www.heterogen-lernen.ch) im Kanton Graubünden. Der Verband der mosaik-Sekundarschulen wiederum unterhält ein Netzwerk von Sekundarschulen, die altersdurchmischte Lerngruppen bilden, individualisiertes Lernen ermöglichen, ein Kurssystem implementieren und Motivation und Selbstständigkeit der Schülerinnen und Schüler fördern (www.mosaiksekundarschulen.ch). Weitere Netzwerke entstehen im Kontext von Schulpreisverleihungen, in deren Rahmen ein netzwerkartiger Austausch über Schul- und Unterrichtsentwicklung möglich ist. Beispiele hierfür sind der „Deutsche Schulpreis“ (www.schulpreis.bosch-stiftung.de) und der „Schweizer Schulpreis“ (www. schweizerschulpreis.ch) oder die im Kanton Zürich durchgeführte Preisverleihung „Schulen lernen von Schulen“ (www.projekt-sls.ch).

\subsection{Rahmenbedingungen}

Eine unterrichtszentrierte Schulentwicklung in Richtung personalisierten Lernens ist eingebettet in Rahmenbedingungen. Als Rahmenbedingungen konkret zu nennen sind insbesondere die folgenden:

- Trägerschaft: öffentlich, privat oder eine Mischform;

- Schulform: Primarschule, Sekundarschule, Gymnasium etc.;

- Zusammensetzung der Schülerinnen und Schüler: soziodemografischer Hintergrund der Schülerinnen und Schüler im Einzugsgebiet;

- finanzielle Ressourcen für Schul- und Unterrichtsentwicklung;

- Gesetze und Verordnungen: juristische Grundlagen für Schule und Unterricht;

- vorhandene Entwicklungszeit (Abbildung 4.22). 


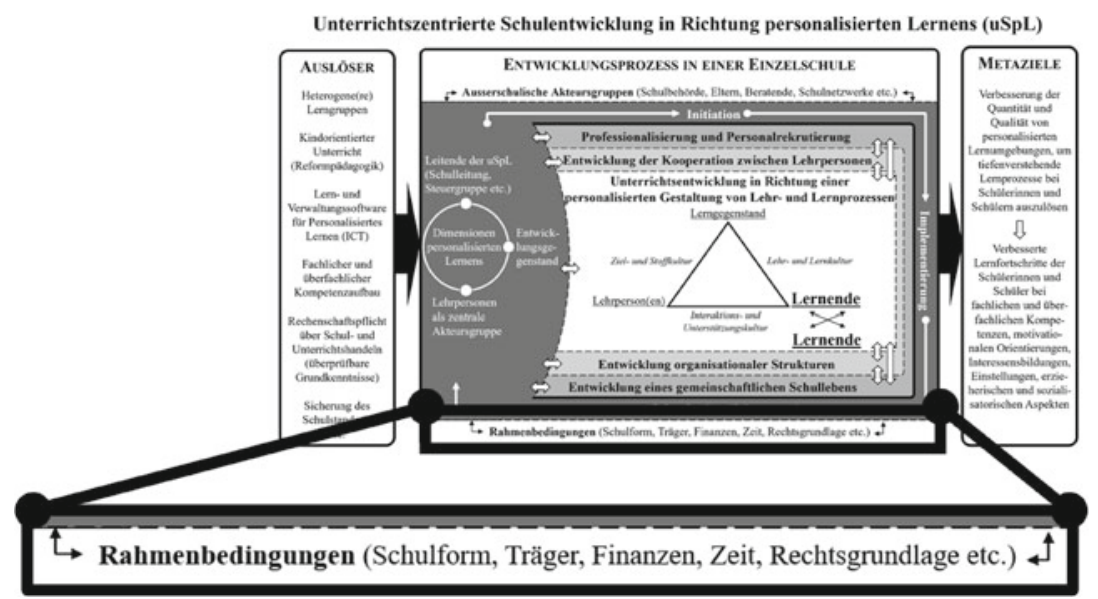

Abbildung 4.22 Rahmenbedingungen im uSpL-Modell (eigene Darstellung)

Innerhalb dieser Rahmenbedingungen bestehen mehr oder weniger große Handlungs- und Autonomiespielräume für einen Entwicklungsprozess (Saalfrank, 2016). Da das Schweizer Bildungssystem föderalistisch organisiert ist, können sich die Rahmenbedingungen von Kanton zu Kanton unterscheiden. Aufgrund der vielen Differenzen bei 26 Kantonen wird jedoch nicht auf kantonale Besonderheiten eingegangen.

Zeit ist eine in der Schulentwicklungsforschung bisher wenig untersuchte Determinante. Es wird davon ausgegangen, dass Veränderungen ungefähr zehn Jahre benötigen, bis sie in den Handlungsroutinen fest verankert sind (u. a. Bohl \& Wacker, 2016; Holtappels, Klemm \& Rolff, 2008; Raggl et al., 2015). Dies dürfte auch für eine unterrichtszentrierte Schulentwicklung in Richtung personalisierten Lernens gelten. Der damit einhergehende Kulturwandel des Lehrens und Lernens und die Veränderung pädagogisch-psychologischer Überzeugungen, Werte und Haltungen der Lehrpersonen brauchen Zeit (Bray \& McClaskey, 2015; Schäfer, Bennemann, Wacker \& Bohl, 2017). Des Weiteren könnte nicht nur die Gesamtlänge des Entwicklungsprozesses, sondern auch die täglich, wöchentlich oder monatlich verfügbare Zeit für Entwicklungstätigkeiten einen Einfluss auf einen erfolgreichen Prozessverlauf haben. $\mathrm{Zu}$ diesem Thema wurden in der einschlägigen Literatur keine Studien gefunden. So würde sich beispielsweise die Frage stellen, ob Kollegien mit mehr verfügbarer Entwicklungszeit wirksamere und nachhaltigere Entwicklungsprozesse gestalten als Kollegien mit weniger 
verfügbarer Entwicklungszeit oder ob es eine Zeitspanne gibt, in der qualitativ hochwertig und dennoch effizient und kostenschonend entwickelt werden kann.

\subsection{Metaziele einer unterrichtszentrierten Schulentwicklung in Richtung personalisierten Lernens}

Das letzte Themenfeld des uSpL-Modells sind die Metaziele, das heißt die übergeordneten Ziele des gesamten Entwicklungsprozesses. Entwicklungstätigkeiten sollen einen verbesserten Lernerfolg der Schülerinnen und Schüler erwirken (Ditton, 2016; Maag Merki, 2008, 2017; Röhrich, 2013; Rolff; Steffens, Bargel \& Höfer, 2016) (Abbildung 4.23).

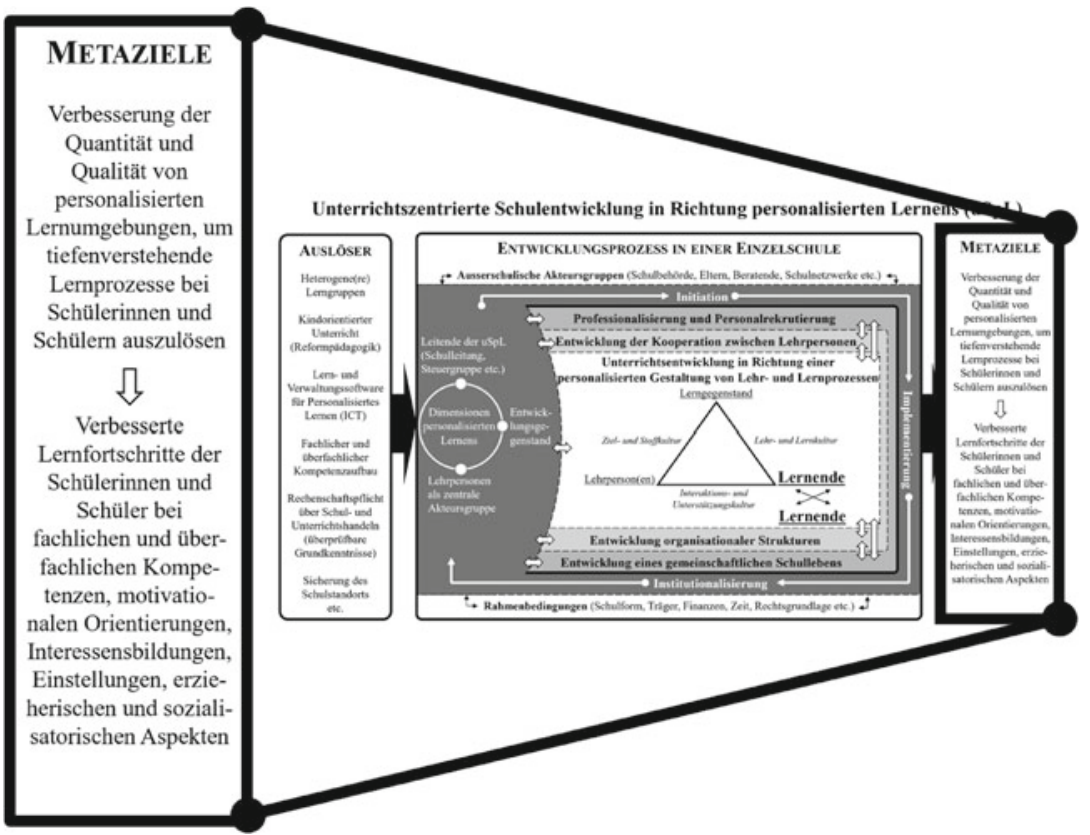

Abbildung 4.23 Metaziele im uSpL-Modell (eigene Darstellung) 
Eine unterrichtszentrierte Schulentwicklung in Richtung personalisierten Lernens zielt darauf ab, die Quantität und die Qualität von personalisierten Lernumgebungen zu verbessern, um auf tiefes Verstehen ausgerichtete Lernprozesse bei Schülerinnen und Schülern auszulösen. Mit dem Entwicklungsfokus auf die Prozessqualitäten von Lernprozessen und auf ein didaktisches Handeln, das die Prozessualitäten auslöst und fördert, sollen Lernfortschritte in fachlichen und überfachlichen Kompetenzen, motivationalen Orientierungen, Interessenbildung, Einstellungen sowie erzieherischen und sozialisatorischen Aspekten erzielt werden.

Im perLen-Forschungsprojekt (Pauli et al., 2017; Petko et al., 2017; Stebler et al., 2018) wurden die 65 Schulen anhand des selbst eingeschätzten Personalisierungsgrads (Gruppendefinition siehe Abschnitt 4.5.1.4) in drei Gruppen unterteilt: sehr stark, stark und moderat personalisierte Schulen. In der ersten und der dritten Gruppe wurden Gruppenunterschiede berechnet, die zwar keinen direkten Anhaltspunkt darauf liefern, dass das jeweilige Konzept personalisierten Lernens diesen Unterschied herbeigeführt hat, jedoch erste Hinweise in diese Interpretationsrichtung geben. Es ergaben sich keine signifikanten Unterschiede in fachlichen Leistungen in Mathematik und Deutsch. In Schulen mit sehr starkem Personalisierungsgrad wurden die Selbstständigkeit und das Verantwortungsbewusstsein bezüglich der Lernprozesse von den Lehrpersonen signifikant positiver eingeschätzt als in Schulen mit moderaten Personalisierungsgrad. Über alle Schulen hinweg zeigte sich, dass die Entwicklung überfachlicher Kompetenzen sowohl von Lehrpersonen als auch von Lernenden positiv, in Schulen mit sehr starkem Personalisierungsgrad sehr positiv, beurteilt worden war (Petko et al., 2017). Zudem gaben die Lehrpersonen an, dass die Schülerinnen und Schüler auch kurz vor Schulabschluss immer noch Freude am Lernen bekunden und schulisches Lernen als nützlich erachten würden. Des Weiteren fühlen sich die Schülerinnen und Schüler gemäß eignen Angaben relativ wohl an ihren Schulen und haben wenig Angst. Die Lehrpersonen beantworteten auch Fragen zur didaktischen Unterrichtsgestaltung und dazu, inwiefern diese sich durch die Einführung der Konzepte personalisierten Lernens verändert hatte. Grundsätzlich schätzten die Lehrpersonen die Unterrichtsqualität höher ein als zuvor. Sie nähmen sich mehr Zeit für einzelne Schülerinnen und Schüler und würden häufiger individuelle Lernziele vereinbaren. Das Erreichen der Lernziele sei nicht schwieriger geworden. Dagegen erachteten die Lehrpersonen die Klassenführung als anspruchsvoller als vor der Personalisierung des Lernens, obwohl die Integration verhaltensauffälliger Schülerinnen und Schüler besser gelinge (Stebler et al., 2017). In einer Befragung von Schulen im Verband der mosaik-Sekundarschulen in der Schweiz, die 
ebenfalls mit Konzepten personalisierten Lernens arbeiten, konnte wie im perLenProjekt nachgewiesen werden, dass die Schülerinnen und Schüler weder Angst vor der Schule haben, noch überfordert sind (André \& Halbheer, 2017).

Zum Thema der Lernzeitnutzung vermochte die Forschungsgruppe WissGem (2016) aufzuzeigen, dass die aktive Lernzeit leistungsstärkerer Schülerinnen und Schüler in offenen Unterrichtssettings am höchsten war. Im geführten Unterricht war dagegen die aktive Lernzeit der leistungsschwächeren Schülerinnen und Schüler am höchsten. Darüber hinaus stellten die Autoren fest, dass die aktive Lernzeit beider Gruppen im offenen Unterrichtssetting dann hoch war, wenn die Schülerinnen und Schüler auswählen konnten, welches Fach sie wann bearbeiten.

Die RAND Corporation (2014) untersuchte die Veränderungen der Lernleistungen von Schülerinnen und Schülern in Schulen mit Konzepten personalisierten Lernens. Die Klassen setzten sich hauptsächlich aus unterprivilegierten Kindern/Jugendlichen zusammen. In einem Abstand von zwei Jahren wurden diese in Mathematik und Lesen getestet. Es ergaben sich deutliche Leistungssteigerungen in beiden Bereichen. Die Leistungen lagen darüber hinaus über dem nationalen Durchschnitt. Die Autoren stellten fest, dass der Leistungszuwachs in Primarschulen (Elementary School) höher war als in Sekundarschulen (Middle School). Auch in einer Begleitevaluation eines Pilotversuchs, bei dem die teilnehmenden Schulen den Entwicklungsauftrag erhalten hatten, personalisierte Lernumgebungen zu entwickeln und einzuführen, lagen die Leistungswerte der Schülerinnen und Schüler in Englisch und Mathematik über dem nationalen Leistungsdurchschnitt. Zudem wurden durchschnittlich weniger Schülerinnen und Schüler von der Schule verwiesen (Tung, Ouimette \& Feldman, 2004).

Zusammengefasst zeigen die derzeit verfügbaren Ergebnisse, dass die personalisierte Gestaltung von Lehr- und Lernprozessen durchaus Potenziale für verbesserte Lernfortschritte aufweist. Jedoch sind die linearen Zusammenhänge zwischen den Unterrichtskonzepten und den verbesserten Lernleistungen insofern kritisch zu beurteilen, als im Lehr- und Lernprozess verschiedene Faktoren den Lernerfolg beeinflussen (Reusser \& Pauli, 2010b). Zum Beispiel ist eine bedeutsamer Einflussfaktor das pädagogische und didaktische Handeln der Lehrperson (Hattie, 2009). Es ist daher denkbar, dass verbesserte Lernleistungen auf besonders motivierte, engagierte und sich intensiv mit qualitätsvollen Lehr- und Lernprozessen auseinandersetzende Lehrpersonen zurückzuführen sind, die möglicherweise häufiger an solchen innovativen Schulen arbeiten als an traditionellen Schulen. 
Open Access Dieses Kapitel wird unter der Creative Commons Namensnennung 4.0 International Lizenz (http://creativecommons.org/licenses/by/4.0/deed.de) veröffentlicht, welche die Nutzung, Vervielfältigung, Bearbeitung, Verbreitung und Wiedergabe in jeglichem Medium und Format erlaubt, sofern Sie den/die ursprünglichen Autor(en) und die Quelle ordnungsgemäß nennen, einen Link zur Creative Commons Lizenz beifügen und angeben, ob Änderungen vorgenommen wurden.

Die in diesem Kapitel enthaltenen Bilder und sonstiges Drittmaterial unterliegen ebenfalls der genannten Creative Commons Lizenz, sofern sich aus der Abbildungslegende nichts anderes ergibt. Sofern das betreffende Material nicht unter der genannten Creative Commons Lizenz steht und die betreffende Handlung nicht nach gesetzlichen Vorschriften erlaubt ist, ist für die oben aufgeführten Weiterverwendungen des Materials die Einwilligung des jeweiligen Rechteinhabers einzuholen. 